\title{
Exercise Training in Patients with Chronic Respiratory Diseases: Are Cardiovascular Comorbidities and Outcomes Taken into Account?-A Systematic Review
}

\author{
Ana Machado 1,2 ${ }^{\mathbb{D}}$, Kirsten Quadflieg ${ }^{1}$, Ana Oliveira ${ }^{2,3,4} \mathbb{D}$, Charly Keytsman ${ }^{1,5}$, \\ Alda Marques 2,6 $\mathbb{D}$, Dominique Hansen 1,5,7 ${ }^{(D)}$ and Chris Burtin 1,5,* \\ 1 REVAL-Rehabilitation Research Center, Faculty of Rehabilitation Sciences, Hasselt University, \\ 3590 Diepenbeek, Belgium \\ 2 Respiratory Research and Rehabilitation Laboratory (Lab3R), School of Health Sciences (ESSUA), \\ University of Aveiro, 3810 Aveiro, Portugal \\ 3 Respiratory Medicine, West Park Healthcare Centre, Toronto, ON M6M 2J5, Canada \\ 4 School of Rehabilitation Sciences, Faculty of Health Sciences, McMaster University, \\ Hamilton, ON L8S 4K1, Canada \\ 5 BIOMED—Biomedical Research Institute, Hasselt University, 3590 Diepenbeek, Belgium \\ 6 Institute of Biomedicine (iBiMED), University of Aveiro, 3810 Aveiro, Portugal \\ 7 Jessa hospital, Heart Centre Hasselt, 3500 Hasselt, Belgium \\ * Correspondence: chris.burtin@uhasselt.be
}

Received: 15 July 2019; Accepted: 10 September 2019; Published: 13 September 2019

\begin{abstract}
Patients with chronic obstructive pulmonary disease (COPD), asthma and interstitial lung diseases (ILD) frequently suffer from cardiovascular comorbidities (CVC). Exercise training is a cornerstone intervention for the management of these conditions, however recommendations on tailoring programmes to patients suffering from respiratory diseases and CVC are scarce. This systematic review aimed to identify the eligibility criteria used to select patients with COPD, asthma or ILD and CVC to exercise programmes; assess the impact of exercise on cardiovascular outcomes; and identify how exercise programmes were tailored to CVC. PubMed, Scopus, Web of Science and Cochrane were searched. Three reviewers extracted the data and two reviewers independently assessed the quality of studies with the Quality Assessment Tool for Quantitative Studies. MetaXL 5.3 was used to calculate the individual and pooled effect sizes (ES). Most studies $(58.9 \%)$ excluded patients with both stable and unstable CVC. In total, 26/42 studies reported cardiovascular outcomes. Resting heart rate was the most reported outcome measure $(n=13)$ and a small statistically significant effect $(E S=-0.23$ ) of exercise training on resting heart rate of patients with COPD was found. No specific adjustments to exercise prescription were described. Few studies have included patients with CVC. There was a lack of tailoring of exercise programmes and limited effects were found. Future studies should explore the effect of tailored exercise programmes on relevant outcome measures in respiratory patients with CVC.
\end{abstract}

Keywords: exercise; chronic lung disease; chronic obstructive pulmonary disease; COPD; asthma; interstitial lung disease; ILD; cardiovascular comorbidities; cardiovascular outcomes

\section{Background}

Chronic obstructive pulmonary disease (COPD), asthma and interstitial lung diseases (ILD) are among the most representative chronic respiratory diseases in the world [1,2]. These diseases affect over 1 billion people and have a significant impact on patients' disability and quality of life $(9.5 \%$ of 
the disability-adjusted life years in 2010 [3]), being a leading contributor to disease burden and one of the top causes of death worldwide (over 3 million deaths in 2016) [2-4].

In recent years, the association between chronic respiratory diseases and cardiovascular diseases has attracted huge interest in clinical research [5]. Cardiovascular diseases (e.g., arterial hypertension, coronary artery disease, congestive heart failure, peripheral vascular disease and pulmonary hypertension) are among the most prevalent and impactful comorbidities in patients with COPD ( $13 \%-68 \%$ of population), asthma ( $3 \%-25 \%$ of population) and ILD ( $8 \%-86 \%$ of population) [6-13]. Namely, they are responsible for further impairing patients' functional status and health-related quality of life, increasing the risk of hospitalization and mortality (hazard ratio 1.1-3.4 [14-17]), and contributing to a higher economic and societal burden, and worse prognosis $[7,9,10,12]$. The need to look beyond the lungs while treating these patients is, therefore, evident [18]. In this regard, a comprehensive assessment and management of these cardiovascular comorbidities with tailored interventions has been recommended $[7,12,19,20]$.

Exercise training is a cornerstone intervention in both pulmonary and cardiac rehabilitation [21,22]. It relieves symptoms and improves functionality, exercise tolerance and health-related quality of life in patients with chronic respiratory and cardiovascular diseases [21,22], and therefore might be a promising intervention for the management of patients with these co-occurring conditions. Nevertheless, studies have shown that these effects are usually reduced in patients suffering from chronic respiratory diseases with accompanying cardiovascular comorbidities compared to those without cardiovascular comorbidities $[7,19,23]$. Moreover, recommendations on how to adjust exercise programmes to co-existing cardiovascular conditions in COPD, asthma and ILD are scarce.

Therefore, in order to inform evidence-based statements, this systematic review aimed to: (i) identify the eligibility criteria in terms of cardiovascular disease that have been used to refer patients with COPD, asthma and ILD for studies investigating the effectiveness of exercise programmes of at least 3 months; (ii) assess the impact of at least 3 months of exercise training on cardiovascular outcomes in these patients; and (iii) identify how the exercise programmes have been tailored to patients' cardiovascular comorbidities.

\section{Methods}

\subsection{Search Strategy}

This systematic review was reported according to the Preferred Reporting Items for Systematic reviews and Meta-analyses (PRISMA) guidelines [24] and was conducted in two phases. Phase 1 identified the eligibility criteria that have been used to select patients with cardiovascular comorbidities in clinical trials investigating the effectiveness of exercise programmes. Phase 2 assessed the impact of exercise training on cardiovascular outcomes, and identified how the exercise programmes have been tailored to patients' cardiovascular comorbidities.

A systematic literature search was performed in May 2019 on the following electronic databases: PubMed, Scopus, Web of Science and Cochrane. The search terms were limited to titles, abstracts and keywords/MeSH terms. The full search strategy is presented in Appendix A.

\subsection{Eligibility Criteria and Study Selection}

For phase 1, studies were included if they (i) studied adult patients with stable COPD, asthma and/or ILD (i.e., 4 weeks without exacerbations); (ii) implemented at least 12 weeks of exercise training (i.e., endurance and/or strength training) as an intervention [25]; (iii) implemented at least 2 directly supervised exercise sessions per week [26]; (iv) were original prospective quantitative studies; and (v) were written in Portuguese, English, French, Dutch or Spanish languages. Retrospective studies, case studies, case series, abstracts and studies involving alternative modalities of exercise (e.g., yoga, tai chi, qigong) were excluded. After removing duplicates, three reviewers (AM, KQ and AO) assessed all the potential studies identified. Studies were selected based on their titles and abstracts. When the title 
and abstract were potentially relevant to the purpose of the review, the full text was read carefully to decide on its inclusion. A fourth reviewer (CB) was consulted to solve any disagreements.

For phase 2, studies included in phase 1 that specified the prevalence of cardiovascular comorbidities (i.e., any cardiovascular condition co-existing with the respiratory disease, identified by doing an objective patients' assessment, checking their medical records or ask patients to self-report their comorbidities) in the baseline characteristics of the population under study and/or reported at least one cardiovascular outcome (i.e., heart rate, systolic and diastolic blood pressure, flow-mediated dilation, pulse-wave velocity, intima thickness of arteria carotid, cardiac function and structure, heart rate variability, ECG analysis and blood lipid profile) were included.

\subsection{Quality Assessment and Data Extraction}

Two reviewers (KQ and $\mathrm{AO}$ ) independently assessed the quality of the studies included in phase 2 with the Quality Assessment Tool for Quantitative Studies, developed by the Effective Public Health Practice Project, Canada [27]. This tool assesses six domains of methodological quality: (i) selection bias; (ii) study design; (iii) confounders; (iv) blinding; (v) data collection methods; and (vi) withdrawals and dropouts [27]. Each domain is rated as "strong", "moderate" or "weak", according to a standardized guide, and the overall rating of the study is determined based on the total number of "strong" and "weak" scores [27].

In phase 1, data regarding the eligibility criteria (i.e., inclusion and exclusion criteria) used to select patients for the study were extracted from all included studies. Afterwards, all conditions that would preclude patients' participation in the exercise programmes, reported either as reasons for inclusion (e.g., absence of severe cardiovascular disease) or exclusion (e.g., presence of severe cardiovascular disease) of these patients, were compiled and reported as exclusion criteria. Additionally, data from the studies included in phase 2 were extracted in a predesigned structured table format comprising the following topics: study (first author, year of publication, country); study design; population (number of participants, diagnosis, age, gender, forced expiratory volume in $1 \mathrm{~s}\left(\mathrm{FEV}_{1}\right)$, forced vital capacity (FVC), diffusing capacity for carbon monoxide (DLCO)); intervention (type and intensity of intervention); duration and frequency (duration of the intervention, duration and frequency of sessions); outcome and outcome measure; and results. For the scope of this review, only cardiovascular outcomes and outcome measures were considered.

\subsection{Data Analysis and Synthesis}

Inter-rater agreement analysis using Cohen's kappa was used to explore the consistency of the quality assessment performed by the two reviewers. The value of Cohen's kappa ranges from 0 to 1 and can be interpreted as slight $(\leq 0.2)$, fair $(0.21-0.4)$, moderate $(0.41-0.6)$, substantial $(0.61-0.8)$, or almost perfect $(\geq 0.81)$ agreement [28]. The statistical analysis was performed using IBM SPSS 24.0 (IBM, Armonk, New York, NY, USA).

Whenever possible, effect sizes (ES) were calculated and a meta-analysis was performed. ES were calculated as Cohens' d based on the Pre/Post means and standard deviations or mean differences and standard deviations, according to the formula of Morris [29], and interpreted as small ( $\geq 0.2)$, medium $(\geq 0.5)$ or large $(\geq 0.8)$ [30]. Meta-analysis was performed on MetaXL 5.3. Pooled effect estimates were calculated with the inverse variance technique assuming a fixed-effects model. The input data were the Cohen's d value of each study and the respective standard error. The output was the pooled Cohen's d value and corresponding confidence intervals. Homogeneity among the studies was evaluated using Cochran's $Q$ test and the $I^{2}$ statistic. 


\section{Results}

\subsection{Study Selection}

The literature search provided a total of 50.970 records. After duplicates removal, 29.756 records were screened for relevant content through title and abstract and 29.248 were excluded. The full text of 508 potentially relevant articles was assessed. From these, 180 articles were included in phase 1 and 42 in phase 2 (Figure 1).

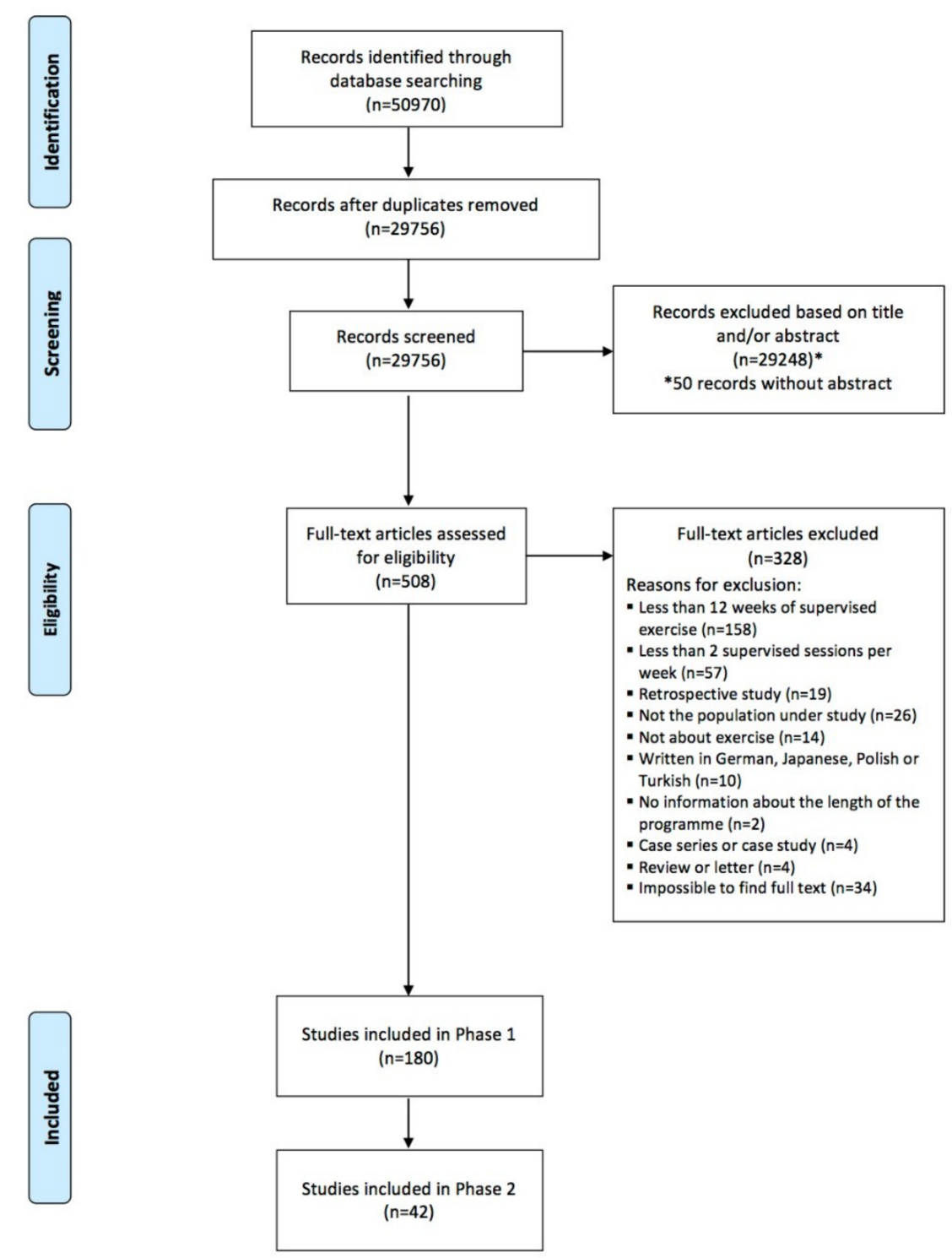

Figure 1. PRISMA flowchart of the included studies.

3.2. Phase 1: Criteria Used to Exclude Patients with Cardiovascular Comorbidities from Exercise Programmes

The 180 studies included were conducted between 1987 and 2019. In total, 156 studies included patients with COPD [23,31-185], 15 studies included patients with asthma [46,52,63,78,185-195] and 16 studies included patients with ILD [87,185,196-209].

Forty-four different exclusion criteria were found in studies with patients with COPD (Figure 2). From these, exercise-limiting conditions [34,36,40,53,60,61,89,92,104,114,125,134,135,138,148,157,166$170,173,174,179,181,183,184](n=27 ; 17.3 \%)$ was the most reported exclusion criterion, followed by general cardiovascular disease $[32,39,44,70,74,75,94,100,103,107,109,112,130,152,153,160,164,171]$ 
( $n=18 ; 11.5 \%)$ and unstable cardiovascular disease $[38,47,53,65,66,98,99,101,108,116-119,133,137,146$, $147,180]$ ( $n=18 ; 11.5 \%)$. Thirty-four $(21.8 \%)$ of the studies $[23,48,50,55,63,67,69,73,77-80,83,84,86,88$, $91,95,97,110,111,115,122,126,127,132,142,149,150,155,161,165,175,185]$ did not report any information regarding the eligibility criteria for cardiovascular comorbidities.

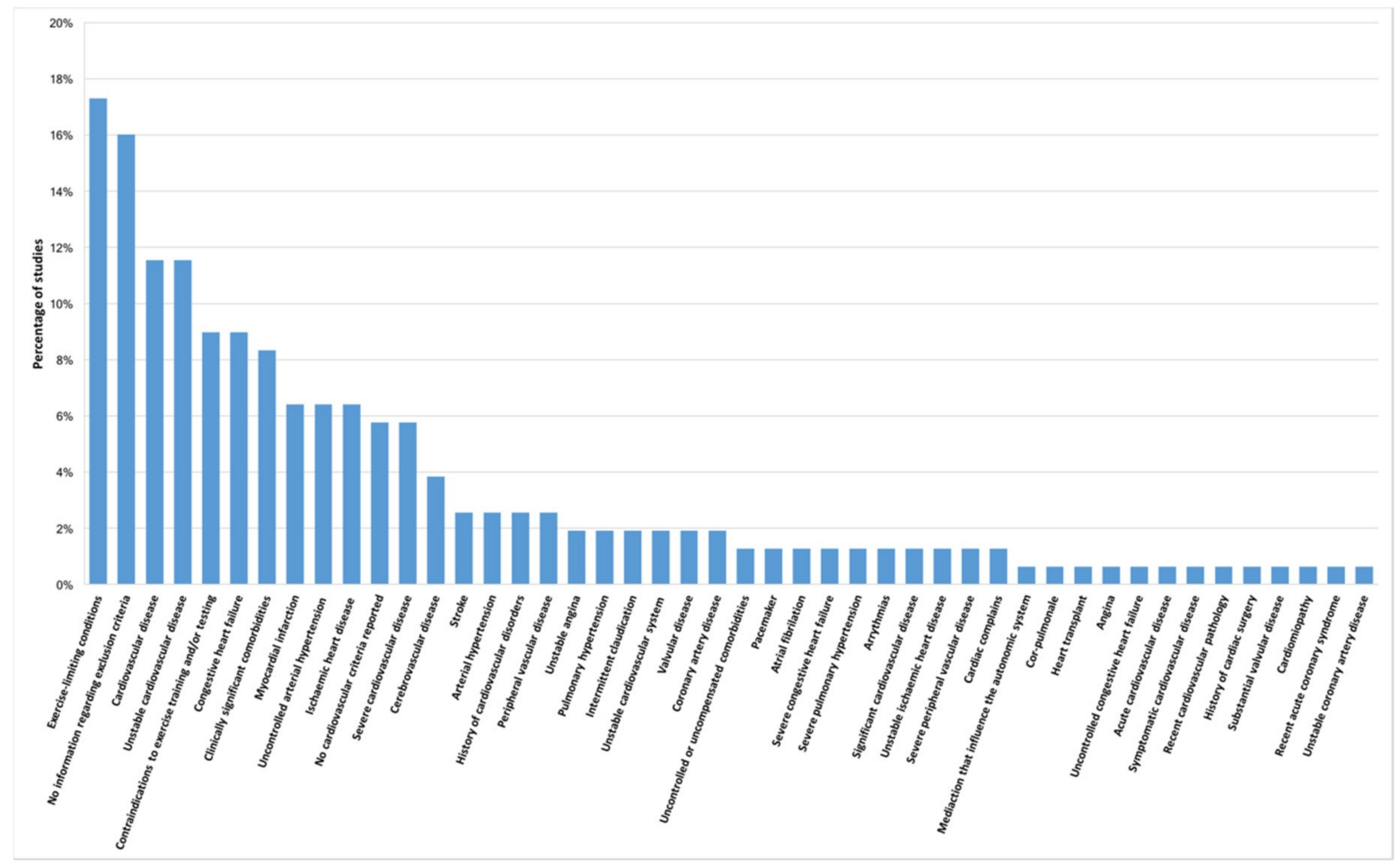

Figure 2. Exclusion criteria reported in studies with patients with chronic obstructive pulmonary disease (COPD) ( $n=156$ studies).

In studies including patients with asthma, 10 different exclusion criteria were found (Figure 3). General cardiovascular disease $[186,191,192](n=3 ; 20.0 \%)$ was the most reported criterion, followed by contraindications to exercise training and/or testing [194,195] $(n=2 ; 13.3 \%)$. Seven $(46.7 \%)$ studies $[63,78,185,187,189,190,193]$ did not report information about exclusion criteria.

Studies in patients with ILD reported 10 different exclusion criteria (Figure 4). From these, unstable cardiovascular disease $[198,199,202,203,208,209](n=6 ; 37.5 \%)$ was the most reported criterion, followed by contraindications to exercise training and/or testing (e.g., unstable angina, recent myocardial infarction or cerebrovascular accident) $[201,204,207](n=3 ; 18.8 \%)$. Four $(25.0 \%)$ studies $[185,197,205,206]$ did not report any information about exclusion criteria.

In general, $22.8 \%$ of the studies $[23,48,50,55,63,67,69,73,77-80,83,84,86,88,91,95,97,110,111$, $115,122,126,127,132,142,149,150,155,161,165,175,185,187,189,190,193,197,205,206]$ did not report information about the eligibility criteria, $18.3 \%$ of the studies $[33,42,54,65,66,72,82,96,98,99,101$, 108,116-119,128,137,141,143,145,146,180,182,194,195,200-203,207-209] only excluded patients with acute or unstable cardiovascular comorbidities that contraindicated exercise training, and 58.9\% of the studies $[31,32,34-41,43-47,49,51-53,56-62,64,68,70,71,74-76,81,85,87,89,90,92-94,100,102-$ 107,109,112-114,120,121,123-125,129-131,133-136,138-140,144,147,148,151-154,156-160,162-164, 166-174,176-179,181,183,184,186,188,191,192,196,198,199,204] excluded both stable and unstable cardiovascular comorbidities. 


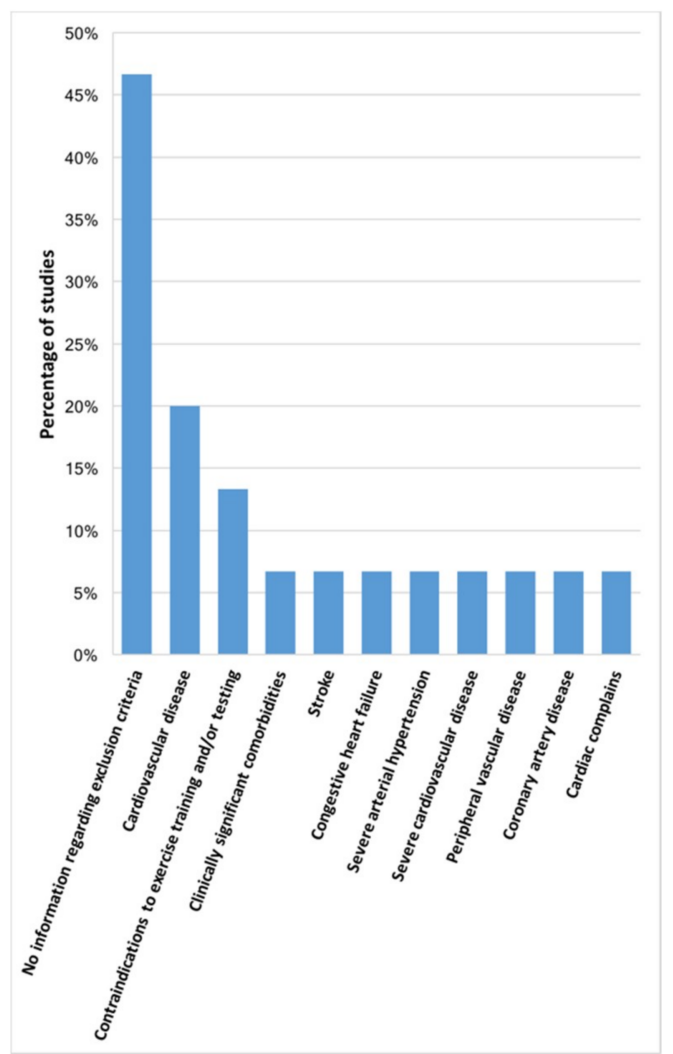

Figure 3. Exclusion criteria reported in studies with patients with asthma ( $n=15$ studies).

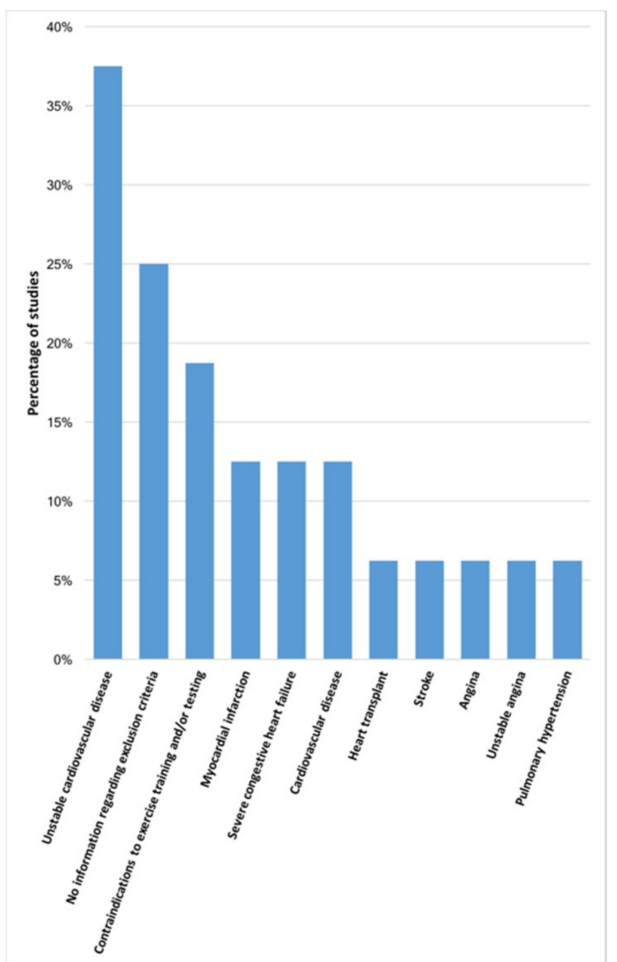

Figure 4. Exclusion criteria reported in studies with patients with interstitial lung diseases (ILD) ( $n=16$ studies). 
3.3. Phase 2: Impact of Exercise Training on Cardiovascular Outcomes and Design of the Exercise Programmes

\subsubsection{Quality Assessment}

Results of the methodological quality assessment are presented in Table 1. Most of the studies $(n=24 ; 57.1 \%)$ were of weak quality. The agreement between the two reviewers was substantial $(\mathrm{k}=0.72 ; 95 \% \mathrm{CI}=0.53-0.91 ; p<0.001$; percentage of agreement $=85.7 \%)$.

\subsubsection{Study Characteristics}

Characteristics of the included studies are shown in Tables 2-4. From the 42 included studies, 32 studies included patients with COPD [33,37,42-44,48,49,53-55,57,59,68,80,85,90,101,104,106-108, $120,121,123,124,128,129,136,146,147,151,167]$, two studies included patients with asthma $[186,189]$ and eight studies included patients with ILD [196,198,201-203,207-209]. Most studies were randomized controlled trials $(n=24 ; 57.1 \%)[37,43,44,49,53,80,85,120,121,123,124,129,146,147,151,167,186,189,196$, 198,202,207-209].

In total, 1704 patients (65.2\% male; data gathered from 34 studies) with a weighted mean age of 65.4 years old and a mean $\mathrm{FEV}_{1}$ of $53.7 \%$ of predicted (data gathered from 36 studies) were enrolled in the included studies.

Only 13 studies in patients with COPD [33,42-44,48,54,55,59,68,85,101,151,167] and six studies in patients with ILD [196,198,203,207-209] specified the presence of patients with cardiovascular comorbidities on the population's baseline characteristics. No studies reporting to include patients with cardiovascular comorbidities were found in asthma. Studies in patients with COPD included patients suffering from arterial hypertension [33,42-44,48,54,68,85,151] (nine studies; 314 patients), cardiovascular diseases $[43,44,48,55,59,85,101,151,167]$ (nine studies; 247 patients), circulatory problems [42-44,85] (four studies; 84 patients), coronary heart disease [42] (one study; 54 patients), congestive heart failure [33,54] (two studies; 10 patients), dyslipidaemia [33,54] (two studies; 11 patients) and ischemic cardiomyopathy [33] (one study; three patients). Studies in patients with ILD included patients suffering from arterial hypertension [196,203,207-209] (five studies; 49 patients), coronary heart disease [207-209] (three studies; 21 patients), congestive heart failure [203] (one study; two patients), pulmonary hypertension [207-209] (three studies; 15 patients) and history of heart disease [198] (one study; one patient). From these, only three studies [196,207,209] conducted in patients with ILD reported cardiovascular outcomes and outcome measures.

Nineteen studies in patients with COPD [37,49,53,57,80,90,104,106-108,120,121,123,124,128,129, $136,146,147]$, two studies in asthma [186,189] and five studies in ILD [196,201,202,207,209] reported cardiovascular outcomes and outcome measures. Studies conducted in patients with COPD presented a large variety of outcome measures, while studies conducted in patients with asthma were mainly focused on blood lipid profile $[187,190](n=2)$ and studies in patients with ILD reported mainly resting heart rate $[196,201,209](n=3)$ and blood pressure $[196,207,209](n=3)$. The most reported outcome measure was resting heart rate $[37,57,90,106,107,121,123,124,129,136,196,201,209](n=13$; $E S=[-0.63 ; 0.11])$. 
Table 1. Quality assessment of the studies included in phase $2(n=42)$ with the Quality Assessment Tool for Quantitative Studies.

\begin{tabular}{|c|c|c|c|c|c|c|c|}
\hline Study & Selection Bias & Study Design & Confounders & Blinding & $\begin{array}{c}\text { Data Collection } \\
\text { Method }\end{array}$ & $\begin{array}{l}\text { Withdrawals and } \\
\text { Drop-Outs }\end{array}$ & Global Rating \\
\hline Cochrane et al., 1990 & 3 & 1 & 1 & 3 & 1 & 3 & 3 \\
\hline Berry et al., 1999 & 2 & 2 & 3 & 3 & 1 & 1 & 3 \\
\hline Foy et al., 2001 & 3 & 1 & 3 & 3 & 1 & 1 & 3 \\
\hline Berry et al., 2003 & 2 & 1 & 1 & 2 & 1 & 1 & 1 \\
\hline Panton et al., 2004 & 2 & 1 & 3 & 3 & 1 & 1 & 3 \\
\hline Marquis et al., 2008 & 2 & 1 & 3 & 1 & 1 & 3 & 3 \\
\hline Averna et al., 2009 & 3 & 1 & 1 & 3 & 1 & 1 & 2 \\
\hline Berry et al., 2010 & 3 & 1 & 1 & 2 & 1 & 1 & 2 \\
\hline Rejbi et al., 2010 & 2 & 1 & 2 & 3 & 1 & 1 & 2 \\
\hline Camillo et al., 2011 & 2 & 1 & 1 & 3 & 1 & 1 & 2 \\
\hline Lan et al., 2011 & 3 & 2 & 2 & 3 & 1 & 3 & 3 \\
\hline Corhay et al., 2012 & 3 & 2 & 3 & 2 & 1 & 2 & 3 \\
\hline Georgiopoulou et al., 2012 & 2 & 2 & NA & 3 & 1 & 1 & 2 \\
\hline Lan et al., 2013 & 2 & 2 & NA & 3 & 1 & 3 & 3 \\
\hline Cheng et al., 2014 & 3 & 2 & NA & 3 & 1 & 3 & 3 \\
\hline Gaunaurd et al., 2014 & 3 & 1 & 1 & 3 & 1 & 1 & 3 \\
\hline Vainshelboim et al., 2014 & 3 & 1 & 1 & 3 & 1 & 1 & 3 \\
\hline Borghi-Silva et al., 2015 & 2 & 1 & 1 & 2 & 1 & 1 & 1 \\
\hline Campos et al., 2015 & 2 & 2 & NA & 3 & 1 & 1 & 2 \\
\hline Leite et al., 2015 & 2 & 1 & 1 & 3 & 1 & 3 & 3 \\
\hline Marcellis et al., 2015 & 2 & 2 & NA & 3 & 1 & 2 & 2 \\
\hline Mkacher et al., 2015 & 2 & 1 & 1 & 3 & 1 & 1 & 2 \\
\hline Spielmanns et al., 2015 & 3 & 1 & 1 & 2 & 1 & 3 & 3 \\
\hline Vainshelboim et al., 2015 & 2 & 1 & 1 & 3 & 1 & 1 & 2 \\
\hline Boström et al., 2016 & 3 & 1 & 1 & 2 & 1 & 1 & 2 \\
\hline Cardoso et al., 2016 & 2 & 1 & 3 & 3 & 1 & 1 & 3 \\
\hline El-Kader et al., 2016 & 3 & 1 & 1 & 3 & 1 & 1 & 3 \\
\hline Engel et al., 2016 & 3 & 1 & 1 & 1 & 1 & 1 & 2 \\
\hline Boeselt et al., 2017 & 3 & 1 & 1 & 3 & 1 & 2 & 3 \\
\hline Kanao et al., 2017 & 3 & 2 & NA & 3 & 1 & 1 & 3 \\
\hline Pacheco et al., 2017 & 2 & 3 & NA & 3 & 1 & 1 & 3 \\
\hline Papp et al., 2017 & 3 & 1 & 3 & 3 & 1 & 2 & 3 \\
\hline
\end{tabular}


Table 1. Cont

\begin{tabular}{|c|c|c|c|c|c|c|c|}
\hline Study & Selection Bias & Study Design & Confounders & Blinding & $\begin{array}{l}\text { Data Collection } \\
\text { Method }\end{array}$ & $\begin{array}{l}\text { Withdrawals and } \\
\text { Drop-Outs }\end{array}$ & Global Rating \\
\hline Vainshelboim et al., 2017 & 2 & 1 & 1 & 3 & 1 & 1 & 2 \\
\hline Vasilopoulou et al., 2017 & 3 & 1 & 3 & 3 & 1 & 1 & 3 \\
\hline Lan et al., 2018 & 3 & 2 & NA & 3 & 1 & 1 & 3 \\
\hline Moezy et al., 2018 & 3 & 1 & 1 & 3 & 1 & 1 & 3 \\
\hline Naz et al., 2018a & 2 & 2 & NA & 3 & 1 & 1 & 2 \\
\hline Naz et al., 2018b & 3 & 1 & 1 & 3 & 1 & 1 & 3 \\
\hline Silva et al., 2018 & 2 & 1 & 1 & 3 & 1 & 1 & 2 \\
\hline Charikiopoulou et al., 2019 & 2 & 2 & 3 & 3 & 1 & 1 & 3 \\
\hline Mekki et al., 2019 & 2 & 1 & 1 & 2 & 1 & 2 & 2 \\
\hline Silva et al., 2019 & 2 & 1 & 1 & 3 & 1 & 1 & 2 \\
\hline
\end{tabular}

Legend: 1 = strong quality; 2 = moderate quality; 3 = weak quality; NA, not applicable. 
Table 2. Characteristics of the studies in patients with COPD included in phase 2 (i.e., studies that specified the prevalence of cardiovascular comorbidities in the baseline characteristics of the population under study and/or reported at least one cardiovascular outcome) $(n=32)$.

\begin{tabular}{|c|c|c|c|c|c|c|}
\hline Study and Country & Study Design & Population & Intervention & $\begin{array}{l}\text { Duration and } \\
\text { Frequency }\end{array}$ & $\begin{array}{l}\text { Cardiovascular Outcomes } \\
\text { and Outcome Measures }\end{array}$ & $\begin{array}{c}\text { Results on Cardiovascular } \\
\text { Outcomes }\end{array}$ \\
\hline $\begin{array}{l}\text { Berry et al., } 1999 \\
\text { United States of } \\
\text { America }\end{array}$ & Non-controlled study & $\begin{array}{l}151 \text { patients with COPD } \\
\text { Mild disease group: } 99\left(54 \sigma^{7} ;\right. \\
67.4 \pm 6.1 \text { years; } \mathrm{FEV}_{1} 68.0 \pm \\
1.2 \% \text { pred) } \\
\text { Arterial hypertension: } n=44 \\
\text { Circulatory problems: } n=14 \\
\text { Coronary heart disease: } n=34 \\
\text { Moderate disease group: } 36 \\
\text { (2207; } \\
68.3 \pm 6.2 \text { years; } \mathrm{FEV}_{1} 41.9 \pm \\
0.7 \% \text { pred) } \\
\text { Arterial hypertension: } n=16 \\
\text { Circulatory problems: } n=5 \\
\text { Coronary heart disease: } n=12 \\
\text { Severe disease group: } 16\left(100^{7} ;\right. \\
66.1 \pm 5.6 \text { years; FEV } 30.1 \pm \\
0.9 \% \text { pred) } \\
\text { Arterial hypertension: } n=7 \\
\text { Circulatory problems: } n=2 \\
\text { Coronary heart disease: } n=8\end{array}$ & $\begin{array}{l}\text { All groups: Aerobic } \\
\text { and strength training } \\
\text { Dyspnoea 3-4 in the } \\
\text { mBorg }\end{array}$ & $\begin{array}{l}\text { All groups: } \\
12 \text { weeks } \\
3 \text { sessions/week } \\
1 \mathrm{~h} / \text { session }\end{array}$ & & \\
\hline $\begin{array}{l}\text { Foy et al., } 2001 \\
\text { United States of } \\
\text { America }\end{array}$ & $\begin{array}{l}\text { Randomized controlled } \\
\text { trial }\end{array}$ & $\begin{array}{l}140 \text { patients with COPD } \\
\text { Short-term intervention group: } \\
70\left(390^{7} ;\right. \\
66.9 \pm 5.9 \text { years; } \mathrm{FEV}_{1} 59.1 \pm \\
17.2 \% \text { pred) } \\
\text { Arterial hypertension: } n=29 \\
\text { Circulatory problems: } n=14 \\
\text { Cardiovascular disease: } n=27 \\
\text { Long-term intervention group: } \\
70\left(390^{7} \text {; }\right. \\
68.4 \pm 6.0 \text { years; FEV } \mathrm{FV}_{1} 57.6 \pm \\
18.4 \% \text { pred) } \\
\text { Arterial hypertension: } n=32 \\
\text { Circulatory problems: } n=9 \\
\text { Cardiovascular disease: } n=24\end{array}$ & $\begin{array}{l}\text { All groups: } \\
\text { Aerobic and strength } \\
\text { training } \\
\text { Dyspnoea 3-4 in the } \\
\text { mBorg }\end{array}$ & $\begin{array}{l}3 \text { sessions/week } \\
55-65 \mathrm{~min} / \text { session } \\
\text { Short-term } \\
\text { intervention group: } \\
12 \text { weeks } \\
\text { Long-term } \\
\text { intervention group: } \\
72 \text { weeks }\end{array}$ & & \\
\hline
\end{tabular}


Table 2. Cont

\begin{tabular}{|c|c|c|c|c|c|c|}
\hline Study and Country & Study Design & Population & Intervention & $\begin{array}{l}\text { Duration and } \\
\text { Frequency }\end{array}$ & $\begin{array}{l}\text { Cardiovascular Outcomes } \\
\text { and Outcome Measures }\end{array}$ & $\begin{array}{c}\text { Results on Cardiovascular } \\
\text { Outcomes }\end{array}$ \\
\hline $\begin{array}{l}\text { Berry et al., } 2003 \\
\text { United States of } \\
\text { America }\end{array}$ & $\begin{array}{l}\text { Randomized controlled } \\
\text { trial }\end{array}$ & $\begin{array}{l}140 \text { patients with COPD } \\
\text { Short-term intervention group: } \\
70\left(390^{7} ;\right. \\
66.9,95 \% \text { CI }(65.5 ; 68.3) \text { years; } \\
\text { FEV } 159.1,95 \% \text { CI (55.0; } \\
63.2) \% \text { pred) } \\
\text { Arterial hypertension: } n=29 \\
\text { Circulatory problems: } n=14 \\
\text { Cardiovascular disease: } n=27 \\
\text { Long-term intervention group: } \\
70\left(390^{7} ;\right. \\
68.4,95 \% \text { CI }(67.0 ; 69.8) \text { years; } \\
\text { FEV } 157.6,95 \% \text { CI }(53.2 ; \\
62.0) \% \text { pred) } \\
\text { Arterial hypertension: } n=32 \\
\text { Circulatory problems: } n=9 \\
\text { Cardiovascular disease: } n=24\end{array}$ & $\begin{array}{l}\text { All groups: } \\
\text { Aerobic and strength } \\
\text { training } \\
\text { Dyspnoea } 3-4 \text { in the } \\
\text { mBorg }\end{array}$ & $\begin{array}{l}3 \text { sessions/week } \\
1 \mathrm{~h} / \text { session } \\
\text { Short-term } \\
\text { intervention group 1: } \\
3 \text { months } \\
\text { Long-term } \\
\text { intervention group: } \\
18 \text { months }\end{array}$ & & \\
\hline \multirow{3}{*}{$\begin{array}{l}\text { Panton et al., } 2004 \\
\text { United States of } \\
\text { America }\end{array}$} & \multirow{3}{*}{$\begin{array}{l}\text { Non-randomized } \\
\text { controlled trial }\end{array}$} & \multirow{3}{*}{$\begin{array}{l}17 \text { patients with COPD } \\
\text { Aerobic training group: } 8\left(20^{7} ;\right. \\
63.0 \pm 8.0 \text { years; } \mathrm{FEV}_{1} 39.5 \pm \\
31.9 \% \text { pred }) \\
\text { Aerobic+strength training } \\
\text { group: } 9\left(60^{7} ;\right. \\
61.0 \pm 7.0 \text { years; } \mathrm{FEV}_{1} 41.9 \pm \\
16.0 \% \text { pred })\end{array}$} & \multirow{3}{*}{$\begin{array}{l}\text { Aerobic training } \\
\text { group: Aerobic } \\
\text { training } \\
50 \%-70 \% \text { of HR } \\
\text { reserve } \\
\text { Aerobic+strength } \\
\text { training group: } \\
\text { Aerobic and strength } \\
\text { training } \\
50 \%-70 \% \text { of } \mathrm{HR} \\
\text { reserve }\end{array}$} & \multirow{3}{*}{$\begin{array}{l}12 \text { weeks } \\
\text { Aerobic training } \\
\text { group: } 2 \\
\text { sessions/week } \\
60 \text { min/session } \\
\text { Aerobic }+ \text { strength } \\
\text { training group: } 4 \\
\text { sessions/week ( } 2 \text { of } \\
\text { each training) } \\
45-60 \text { min/session }\end{array}$} & Rate pressure product & $\begin{array}{l}\text { Aerobic training group: Pre } \\
177.0 \pm 29.0 \text { vs. Post } 186.0 \pm \\
30.0, p>0.05 \\
E S=0.31 \\
\text { Aerobic+strength training } \\
\text { group: Pre } 195.0 \pm 35.0 \text { vs. } \\
\text { Post } 199.0 \pm 35.0, p>0.05 \\
E S=0.11\end{array}$ \\
\hline & & & & & $\begin{array}{l}\text { Total blood cholesterol } \\
(\mathrm{mg} / \mathrm{dl})\end{array}$ & $\begin{array}{l}\text { Aerobic training group: Pre } \\
217.0 \pm 46.0 \text { vs. Post } 217.0 \pm \\
46.0, p>0.05 \\
E S=0.00 \\
\text { Aerobic+strength training } \\
\text { group: Pre } 201.0 \pm 34.0 \text { vs. } \\
\text { Post } 193.0 \pm 23.0, p>0.05 \\
E S=-0.28\end{array}$ \\
\hline & & & & & Cholesterol - HDL (mg/dl) & $\begin{array}{l}\text { Aerobic training group: Pre } \\
62.0 \pm 21.0 \text { vs. Post } 62.0 \pm \\
20.0, p>0.05 \\
E S=0.00 \\
\text { Aerobic+strength training } \\
\text { group: Pre } 55.0 \pm 16.0 \text { vs. } \\
\text { Post } 53.0 \pm 12.0, p>0.05 \\
E S=-0.14\end{array}$ \\
\hline
\end{tabular}


Table 2. Cont

\begin{tabular}{|c|c|c|c|c|c|c|}
\hline Study and Country & Study Design & Population & Intervention & $\begin{array}{l}\text { Duration and } \\
\text { Frequency }\end{array}$ & $\begin{array}{l}\text { Cardiovascular Outcomes } \\
\text { and Outcome Measures }\end{array}$ & $\begin{array}{c}\text { Results on Cardiovascular } \\
\text { Outcomes }\end{array}$ \\
\hline & & & & & Cholesterol - LDL (mg/dl) & $\begin{array}{l}\text { Aerobic training group: Pre } \\
129.0 \pm 34.0 \text { vs. Post } 132.0 \pm \\
35.0, p>0.05 \\
E S=0.09 \\
\text { Aerobic+strength training } \\
\text { group: Pre } 122.0 \pm 21.0 \text { vs. } \\
\text { Post } 118.0 \pm 15.0, p>0.05 \\
E S=-0.22\end{array}$ \\
\hline & & & & & $\begin{array}{l}\text { Cholesterol - Triglycerides } \\
(\mathrm{mg} / \mathrm{dl})\end{array}$ & $\begin{array}{l}\text { Aerobic training group: Pre } \\
151.0 \pm 65.0 \text { vs. Post } 185.0 \pm \\
87.0, p<0.05 \\
E S=0.44 \\
\text { Aerobic+strength training } \\
\text { group: Pre } 141.0 \pm 132.0 \text { vs. } \\
\text { Post } 135.0 \pm 73.0, p>0.05 \\
E S=-0.06\end{array}$ \\
\hline & & & & & Total cholesterol/HDL ratio & $\begin{array}{l}\text { Aerobic training group: Pre } \\
3.8 \pm 1.1 \text { vs. Post } 3.9 \pm 1.1, \\
p>0.05 \\
E S=0.09 \\
\text { Aerobic+strength training } \\
\text { group: Pre } 3.8 \pm 0.8 \text { vs. } \\
\text { Post } 3.8 \pm 0.8, p>0.05 \\
E S=0.00\end{array}$ \\
\hline \multirow{2}{*}{$\begin{array}{l}\text { Marquis et al., } 2008 \\
\text { Canada }\end{array}$} & \multirow{2}{*}{$\begin{array}{l}\text { Randomized controlled } \\
\text { trial }\end{array}$} & \multirow{2}{*}{ 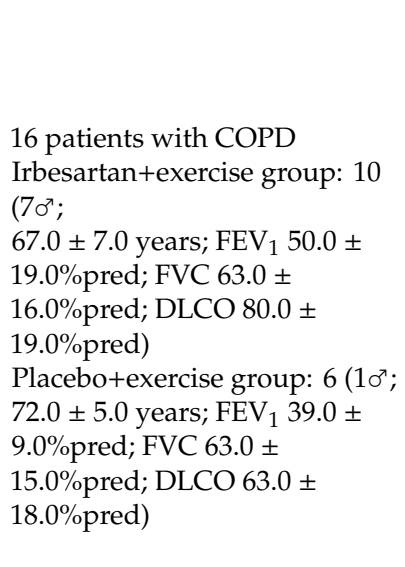 } & \multirow{2}{*}{$\begin{array}{l}\text { All groups: } \\
\text { Aerobic training } \\
80 \% \text { of WRmax }\end{array}$} & \multirow{2}{*}{$\begin{array}{l}\text { All groups: } \\
12 \text { weeks } \\
3 \text { sessions/week } \\
30 \text { min/session }\end{array}$} & $\begin{array}{l}\text { Systolic blood pressure at } \\
\text { rest (mmHg) }\end{array}$ & $\begin{array}{l}\text { Irbesartan+exercise group: } \\
\text { Pre } 151.0 \pm 19.0 \text { vs. Post } \\
131.0 \pm 18.0, p<0.05 \\
E S=-1.08 \\
\text { Placebo+exercise group: } \\
\text { Pre } 140.0 \pm 15.0 \text { vs. Post } \\
136.0 \pm 15.0, p>0.05 \\
E S=-0.27\end{array}$ \\
\hline & & & & & $\begin{array}{l}\text { Mean systolic blood } \\
\text { pressure during } 24 \mathrm{~h} \\
(\mathrm{mmHg})\end{array}$ & $\begin{array}{l}\text { Irbesartan+exercise group: } \\
\text { Pre } 135.0 \pm 9.0 \text { vs. Post } \\
126.0 \pm 12.0, p<0.01 \\
E S=-0.85 \\
\text { Placebo+exercise group: } \\
\text { Pre } 130.0 \pm 14.0 \text { vs. Post } \\
128.0 \pm 8.0, p>0.05 \\
E S=-0.18\end{array}$ \\
\hline
\end{tabular}


Table 2. Cont.

\begin{tabular}{|c|c|c|c|c|c|c|}
\hline Study and Country & Study Design & Population & Intervention & $\begin{array}{c}\text { Duration and } \\
\text { Frequency }\end{array}$ & $\begin{array}{c}\text { Cardiovascular Outcomes } \\
\text { and Outcome Measures }\end{array}$ & $\begin{array}{c}\text { Results on Cardiovascular } \\
\text { Outcomes }\end{array}$ \\
\hline & & & & & $\begin{array}{l}\text { Mean systolic blood } \\
\text { pressure at daytime } \\
(\mathrm{mmHg})\end{array}$ & $\begin{array}{l}\text { Irbesartan+exercise group: } \\
\text { Pre } 139.0 \pm 11.0 \text { vs. Post } \\
129.0 \pm 15.0, p<0.01 \\
E S=-0.76 \\
\text { Placebo+exercise group: } \\
\text { Pre } 130.0 \pm 14.0 \text { vs. Post } \\
131.0 \pm 8.0, p>0.05 \\
E S=0.09\end{array}$ \\
\hline & & & & & $\begin{array}{l}\text { Mean systolic blood } \\
\text { pressure at nighttime } \\
(\mathrm{mmHg})\end{array}$ & $\begin{array}{l}\text { Irbesartan+exercise group: } \\
\text { Pre } 125.0 \pm 8.0 \text { vs. Post } \\
121.0 \pm 10.0, p>0.05 \\
E S=-0.44 \\
\text { Placebo+exercise group: } \\
\text { Pre } 128.0 \pm 16.0 \text { vs. Post } \\
121.0 \pm 9.0, p>005 \\
E S=-0.54\end{array}$ \\
\hline & & & & & $\begin{array}{l}\text { Diastolic blood pressure at } \\
\text { rest }(\mathrm{mmHg})\end{array}$ & $\begin{array}{l}\text { Irbesartan+exercise group: } \\
\text { Pre } 78.0 \pm 8.0 \text { vs. Post } 71.0 \\
\pm 10.0, p<0.05 \\
E S=-0.77 \\
\text { Placebo+exercise group: } \\
\text { Pre } 72.0 \pm 8.0 \text { vs. Post } 68.0 \\
\pm 10.0, p>0.05 \\
E S=-0.44\end{array}$ \\
\hline & & & & & $\begin{array}{l}\text { Mean diastolic blood } \\
\text { pressure during } 24 \mathrm{~h} \\
(\mathrm{mmHg})\end{array}$ & $\begin{array}{l}\text { Irbesartan+exercise group: } \\
\text { Pre } 76.0 \pm 9.0 \text { vs. Post } 72.0 \\
\pm 8.0, p<0.05 \\
E S=-0.47 \\
\text { Placebo+exercise group: } \\
\text { Pre } 70.0 \pm 3.0 \text { vs. Post } 70.0 \\
\pm 8.0, p>0.05 \\
E S=0.00\end{array}$ \\
\hline & & & & & $\begin{array}{l}\text { Mean diastolic blood } \\
\text { pressure at daytime } \\
(\mathrm{mmHg})\end{array}$ & $\begin{array}{l}\text { Irbesartan+exercise group: } \\
\text { Pre } 80.0 \pm 11.0 \text { vs. Post } 74.0 \\
\pm 10.0, p<0.05 \\
E S=-0.84 \\
\text { Placebo+exercise group: } \\
\text { Pre } 71.0 \pm 2.0 \text { vs. Post } 72.0 \\
\pm 7.0, p>0.05 \\
E S=0.19\end{array}$ \\
\hline
\end{tabular}


Table 2. Cont.

\begin{tabular}{|c|c|c|c|c|c|c|}
\hline Study and Country & Study Design & Population & Intervention & $\begin{array}{c}\text { Duration and } \\
\text { Frequency }\end{array}$ & $\begin{array}{l}\text { Cardiovascular Outcomes } \\
\text { and Outcome Measures }\end{array}$ & $\begin{array}{c}\text { Results on Cardiovascular } \\
\text { Outcomes }\end{array}$ \\
\hline & & & & & $\begin{array}{l}\text { Mean diastolic blood } \\
\text { pressure at nighttime } \\
(\mathrm{mmHg})\end{array}$ & $\begin{array}{l}\text { Irbesartan+exercise group: } \\
\text { Pre } 68.0 \pm 6.0 \text { vs. Post } 67.0 \\
\pm 7.0, p>0.05 \\
E S=-0.15 \\
\text { Placebo+exercise group: } \\
\text { Pre } 66.0 \pm 6.0 \text { vs. Post } 65.0 \\
\pm 8.0, p>0.05 \\
E S=-0.14\end{array}$ \\
\hline & & & & & $\begin{array}{l}\text { Standard deviation of all } \\
\text { NN intervals (ms) }\end{array}$ & $\begin{array}{l}\text { Irbesartan+exercise group: } \\
\text { Pre } 102.0 \pm 28.0 \text { vs. Post } \\
144.0 \pm 36.0, p>0.05 \\
E S=1.30 \\
\text { Placebo+exercise group: } \\
\text { Pre } 121.0 \pm 27.0 \text { vs. Post } \\
113.0 \pm 38.0, p>0.05 \\
E S=-0.24\end{array}$ \\
\hline & & & & & $\begin{array}{l}\text { Adjacent normal-to-normal } \\
(\mathrm{NN}) \text { intervals differing by } \\
\text { more than } 50 \mathrm{~ms}(\%)\end{array}$ & $\begin{array}{l}\text { Irbesartan+exercise group: } \\
\text { Pre } 9.0 \pm 9.0 \text { vs. Post } 9.0 \pm \\
8.0, p>0.05 \\
E S=0.00 \\
\text { Placebo+exercise group: } \\
\text { Pre } 10.0 \pm 9.0 \text { vs. Post } 10.0 \\
\pm 8.0, p>0.05 \\
E S=0.00\end{array}$ \\
\hline & & & & & $\begin{array}{l}\text { Square root of the mean } \\
\text { squared differences of } \\
\text { successive NN intervals } \\
(\mathrm{ms})\end{array}$ & $\begin{array}{l}\text { Irbesartan+exercise group: } \\
\text { Pre } 30.0 \pm 12.0 \text { vs. Post } 29.0 \\
\pm 11.0, p>0.05 \\
E S=-0.09 \\
\text { Placebo+exercise group: } \\
\text { Pre } 31.0 \pm 11.0 \text { vs. Post } 30.0 \\
\pm 10.0, p>0.05 \\
E S=-0.10\end{array}$ \\
\hline & & & & & Very low frequency (ms) & $\begin{array}{l}\text { Irbesartan+exercise group: } \\
\text { Pre } 3.3 \pm 0.2 \text { vs. Post } 3.3 \pm \\
0.2, p>0.05 \\
E S=0.00 \\
\text { Placebo+exercise group: } \\
\text { Pre } 3.1 \pm 0.2 \text { vs. Post } 3.1 \pm \\
0.5, p>0.05 \\
E S=0.07\end{array}$ \\
\hline
\end{tabular}


Table 2. Cont.

\begin{tabular}{|c|c|c|c|c|c|c|}
\hline Study and Country & Study Design & Population & Intervention & $\begin{array}{l}\text { Duration and } \\
\text { Frequency }\end{array}$ & $\begin{array}{l}\text { Cardiovascular Outcomes } \\
\text { and Outcome Measures }\end{array}$ & $\begin{array}{c}\text { Results on Cardiovascular } \\
\text { Outcomes }\end{array}$ \\
\hline & & & & & Low frequency (ms) & $\begin{array}{l}\text { Irbesartan+exercise group: } \\
\text { Pre } 2.9 \pm 0.3 \text { vs. Post } 2.9 \pm \\
0.3, p>0.05 \\
E S=-0.03 \\
\text { Placebo+exercise group: } \\
\text { Pre } 2.8 \pm 0.4 \text { vs. Post } 2.8 \pm \\
0.4, p>0.05 \\
E S=0.02\end{array}$ \\
\hline & & & & & High frequency (ms) & $\begin{array}{l}\text { Irbesartan+exercise group: } \\
\text { Pre } 2.4 \pm 0.3 \text { vs. Post } 2.4 \pm \\
0.4, p>0.05 \\
E S=-0.03 \\
\text { Placebo+exercise group: } \\
\text { Pre } 2.4 \pm 0.4 \text { vs. Post } 2.4 \pm \\
0.5, p>0.05 \\
E S=-0.12\end{array}$ \\
\hline & & & & & $\begin{array}{l}\text { Low frequency/High } \\
\text { frequency ratio }\end{array}$ & $\begin{array}{l}\text { Irbesartan+exercise group: } \\
\text { Pre } 3.0 \pm 1.3 \text { vs. Post } 3.1 \pm \\
1.3, p>0.05 \\
E S=0.08 \\
\text { Placebo+exercise group: } \\
\text { Pre } 2.1 \pm 1.0 \text { vs. Post } 2.4 \pm \\
1.0, p>0.05 \\
E S=0.27\end{array}$ \\
\hline \multirow{3}{*}{$\begin{array}{l}\text { Averna et al., } 2009 \\
\text { Italy }\end{array}$} & \multirow{3}{*}{$\begin{array}{l}\text { Randomized controlled } \\
\text { trial }\end{array}$} & \multirow{3}{*}{$\begin{array}{l}56 \text { patients with COPD }\left(290^{7}\right. \\
69.0 \pm 5.0 \text { years; } \mathrm{FEV}_{1} 82.0 \pm \\
16.6 \% \text { pred; FVC } 91.0 \pm \\
17.4 \% \text { pred })\end{array}$} & \multirow{3}{*}{$\begin{array}{l}\text { Aerobic and strength } \\
\text { training } \\
40 \%-50 \% \text { of } \mathrm{HR} \\
\text { reserve } \\
50 \% \text { of } 1 \mathrm{RM}\end{array}$} & \multirow{3}{*}{$\begin{array}{l}12 \text { weeks } \\
3 \text { sessions/week } \\
60 \mathrm{~min} / \mathrm{session}\end{array}$} & HR at rest (bpm) & $\begin{array}{l}\text { Pre } 65.0 \pm 10.0 \text { vs. Post } 65.0 \\
\pm 9.0, \mathrm{p}=0.64 \\
E S=0.00\end{array}$ \\
\hline & & & & & $\begin{array}{l}\text { Systolic blood pressure at } \\
\text { rest }(\mathrm{mmHg})\end{array}$ & $\begin{array}{l}\text { Pre } 137.0 \pm 12.0 \text { vs. Post } \\
131.0 \pm 12.0, \mathrm{p}=0.001 \\
E S=-0.70\end{array}$ \\
\hline & & & & & $\begin{array}{l}\text { Diastolic blood pressure at } \\
\text { rest }(\mathrm{mmHg})\end{array}$ & $\begin{array}{l}\text { Pre } 84.0 \pm 6.0 \text { vs. Post } 80.0 \\
\pm 7.0, p=0.001 \\
E S=-0.61\end{array}$ \\
\hline $\begin{array}{l}\text { Berry et al., } 2010 \\
\text { United States of } \\
\text { America }\end{array}$ & $\begin{array}{l}\text { Randomized controlled } \\
\text { trial }\end{array}$ & $\begin{array}{l}89 \text { patients with COPD }\left(480^{7} ;\right. \\
66.0 \pm 10.0 \text { years; } \mathrm{FEV}_{1} 53.0 \pm \\
18.5 \% \text { pred }) \\
\text { Arterial hypertension: } n=47 \\
\text { Circulatory problems: } n=17 \\
\text { Cardiovascular disease: } n=39\end{array}$ & $\begin{array}{l}\text { Aerobic and strength } \\
\text { training } \\
\text { Dyspnoea } 3-5 \text { in the } \\
\text { mBorg }\end{array}$ & $\begin{array}{l}12 \text { weeks } \\
3 \text { sessions/week } \\
1 \mathrm{~h} / \text { session }\end{array}$ & & \\
\hline
\end{tabular}


Table 2. Cont.

\begin{tabular}{|c|c|c|c|c|c|c|}
\hline Study and Country & Study Design & Population & Intervention & $\begin{array}{l}\text { Duration and } \\
\text { Frequency }\end{array}$ & $\begin{array}{l}\text { Cardiovascular Outcomes } \\
\text { and Outcome Measures }\end{array}$ & $\begin{array}{c}\text { Results on Cardiovascular } \\
\text { Outcomes }\end{array}$ \\
\hline $\begin{array}{l}\text { Rejbi et al., } 2010 \\
\text { Tunisia }\end{array}$ & $\begin{array}{l}\text { Non-randomized } \\
\text { controlled trial }\end{array}$ & $\begin{array}{l}26 \text { patients with COPD }(61.0 \pm \\
4.0 \text { years; FEV } 148.9 \pm \\
11.3 \% \text { pred; FVC } 58.8 \pm \\
9.8 \% \text { pred })\end{array}$ & $\begin{array}{l}\text { Pulmonary } \\
\text { rehabilitation } \\
\text { HR of the gas } \\
\text { exchange threshold }\end{array}$ & $\begin{array}{l}3 \text { months } \\
3 \text { sessions/week } \\
45 \text { min/session }\end{array}$ & $\mathrm{HR}$ at rest (bpm) & $\begin{array}{l}\text { Pre } 75.6 \pm 13.9 \text { vs. Post } 76.5 \\
\pm 14.0, p>0.05 \\
E S=0.06\end{array}$ \\
\hline \multirow{4}{*}{$\begin{array}{l}\text { Camillo et al., } 2011 \\
\text { Brazil }\end{array}$} & \multirow{4}{*}{$\begin{array}{l}\text { Randomized controlled } \\
\text { trial }\end{array}$} & \multirow{4}{*}{$\begin{array}{l}40 \text { patients with COPD } \\
\text { High-intensity group: } 20\left(10 \sigma^{7} ;\right. \\
67.0 \pm 7.0 \text { years; } \mathrm{FEV}_{1} 40.0 \pm \\
13.0 \% \text { pred }) \\
\text { Low-intensity group: } 20\left(110^{7} ;\right. \\
65.0 \pm 10.0 \text { years; } \mathrm{FEV}_{1} 39.0 \pm \\
14.0 \% \text { pred })\end{array}$} & \multirow{4}{*}{$\begin{array}{l}\text { High-intensity group: } \\
\text { Aerobic and strength } \\
\text { training } \\
60 \% \text { of WRmax } \\
75 \% \text { of average } \\
\text { walking speed in the } \\
6 \mathrm{MWT} \\
70 \% \text { of } 1 \mathrm{RM} \\
\text { Low-intensity group: } \\
\text { Strength training }\end{array}$} & \multirow{4}{*}{$\begin{array}{l}\text { All groups: } \\
12 \text { weeks } \\
3 \text { sessions/week } \\
1 \text { h/session }\end{array}$} & $\begin{array}{l}\text { Standard deviation of N-N } \\
\text { intervals (ms) }\end{array}$ & $\begin{array}{l}\text { High-intensity group: Pre } \\
29.0 \pm 15.0 \text { vs. Post } 36.0 \pm \\
19.0, p<0.05 \\
E S=0.41 \\
\text { Low-intensity group: Pre } \\
25.0 \pm 12.0 \text { vs. Post } 22.0 \pm \\
10.0, p>0.05 \\
E S=-0.27\end{array}$ \\
\hline & & & & & $\begin{array}{l}\text { Square root of the mean } \\
\text { squared difference of the } \\
\text { successive N-N intervals } \\
\text { (ms) }\end{array}$ & $\begin{array}{l}\text { High-intensity group: Pre } \\
22.0 \pm 14.0 \text { vs. Post } 28.0 \pm \\
22.0, p<0.05 \\
E S=0.33 \\
\text { Low-intensity group: Pre } \\
22.0 \pm 22.0 \text { vs. Post } 19.0 \pm \\
14.0, p>0.05 \\
E S=-0.16\end{array}$ \\
\hline & & & & & $\begin{array}{l}\text { Low frequency in supine } \\
(\%)\end{array}$ & $\begin{array}{l}\text { High-intensity group: Pre } \\
44.0 \pm 15.0 \text { vs. Post } 42.0 \pm \\
24.0, p>0.05 \\
E S=-0.10 \\
\text { Low-intensity group: Pre } \\
48.0 \pm 19.0 \text { vs. Post } 43.0 \pm \\
19.0, p>0.05 \\
E S=-0.26\end{array}$ \\
\hline & & & & & $\begin{array}{l}\text { Low frequency in } \\
\text { orthostatic }(\%)\end{array}$ & $\begin{array}{l}\text { High-intensity group: Pre } \\
55.0 \pm 21.0 \text { vs. Post } 50.0 \pm \\
20.0, p>0.05 \\
E S=-0.24 \\
\text { Low-intensity group: Pre } \\
58.0 \pm 15.0 \text { vs. Post } 62.0 \pm \\
20.0, p>0.05 \\
E S=0.23\end{array}$ \\
\hline
\end{tabular}


Table 2. Cont.

\begin{tabular}{|c|c|c|c|c|c|c|}
\hline Study and Country & Study Design & Population & Intervention & $\begin{array}{l}\text { Duration and } \\
\text { Frequency }\end{array}$ & $\begin{array}{l}\text { Cardiovascular Outcomes } \\
\text { and Outcome Measures }\end{array}$ & $\begin{array}{c}\text { Results on Cardiovascular } \\
\text { Outcomes }\end{array}$ \\
\hline & & \multirow[t]{4}{*}{ ) } & & & $\begin{array}{l}\text { High frequency in supine } \\
(\%)\end{array}$ & $\begin{array}{l}\text { High-intensity group: Pre } \\
56.0 \pm 15.0 \text { vs. Post } 58.0 \pm \\
24.0, p>0.05 \\
E S=0.10 \\
\text { Low-intensity group: Pre } \\
51.0 \pm 19.0 \text { vs. Post } 56.0 \pm \\
19.0, p>0.05 \\
E S=0.26\end{array}$ \\
\hline & & & & & $\begin{array}{l}\text { High frequency in } \\
\text { orthostatic (\%) }\end{array}$ & $\begin{array}{l}\text { High-intensity group: Pre } \\
44.0 \pm 21.0 \text { vs. Post } 50.0 \pm \\
20.0, p>0.05 \\
E S=0.29 \\
\text { Low-intensity group: Pre } \\
41.0 \pm 15.0 \text { vs. Post } 37.0 \pm \\
20.0, p>0.05 \\
E S=-0.23\end{array}$ \\
\hline & & & & & $\begin{array}{l}\text { Low frequency/High } \\
\text { frequency ratio in supine }\end{array}$ & $\begin{array}{l}\text { High-intensity group: Pre } \\
0.9 \pm 0.8 \text { vs. Post } 1.3 \pm 1.5, p \\
>0.05 \\
E S=0.60 \\
\text { Low-intensity group: Pre } \\
1.2 \pm 0.9 \text { vs. Post } 1.1 \pm 1.2, p \\
>0.05 \\
E S=-0.09\end{array}$ \\
\hline & & & & & $\begin{array}{l}\text { Low frequency/High } \\
\text { frequency ratio in } \\
\text { orthostatic }\end{array}$ & $\begin{array}{l}\text { High-intensity group: Pre } \\
2.3 \pm 3.1 \text { vs. Post } 1.3 \pm 0.9, p \\
>0.05 \\
E S=-0.44 \\
\text { Low-intensity group: Pre } \\
1.7 \pm 1.0 \text { vs. Post } 2.8 \pm 2.8, p \\
>0.05 \\
E S=0.52\end{array}$ \\
\hline $\begin{array}{l}\text { Lan et al., } 2011 \\
\text { Taiwan }\end{array}$ & Non-controlled study & 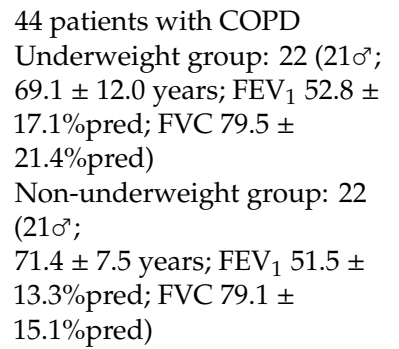 & $\begin{array}{l}\text { All groups: } \\
\text { Pulmonary } \\
\text { rehabilitation } \\
50 \%-75 \% \text { of } \mathrm{VO} 2 \text { peak }\end{array}$ & $\begin{array}{l}\text { All groups: } \\
12 \text { weeks } \\
2 \text { sessions/week } \\
40-50 \mathrm{~min} / \text { session }\end{array}$ & HR at rest (bpm) & $\begin{array}{l}\text { Underweight group: Pre } \\
85.2 \pm 13.0 \text { vs. Post } 83.1 \pm \\
11.7, \mathrm{p}=0.315 \\
E S=-0.17 \\
\text { Non-underweight group: } \\
\text { Pre } 88.2 \pm 11.6 \text { vs. Post } 86.0 \\
\pm 10.8, \mathrm{p}=0.029 \\
E S=-0.20\end{array}$ \\
\hline
\end{tabular}


Table 2. Cont

\begin{tabular}{|c|c|c|c|c|c|c|}
\hline Study and Country & Study Design & Population & Intervention & $\begin{array}{l}\text { Duration and } \\
\text { Frequency }\end{array}$ & $\begin{array}{l}\text { Cardiovascular Outcomes } \\
\text { and Outcome Measures }\end{array}$ & $\begin{array}{c}\text { Results on Cardiovascular } \\
\text { Outcomes }\end{array}$ \\
\hline $\begin{array}{l}\text { Corhay et al., } 2012 \\
\text { Belgium }\end{array}$ & Non-controlled study & $\begin{array}{l}140 \text { patients with COPD } \\
<65 \text { years group: } 69\left(42 \sigma^{7} ;\right. \\
57.6 \pm 5.2 \text { years; } \mathrm{FEV}_{1} 38.1 \pm \\
10.8 \% \text { pred }) \\
\text { Cardiovascular disease: } n=19 \\
65-74 \text { years group: } 50\left(360^{7} ;\right. \\
69.5 \pm 2.6 \text { years; } \mathrm{FEV}_{1} 39.5 \pm \\
11.7 \% \text { pred) } \\
\text { Cardiovascular disease: } n=23 \\
\geq 75 \text { years group: } 21\left(170^{7} ;\right. \\
77.4 \pm 2.5 \text { years; } \mathrm{FEV}_{1} 39.9 \pm \\
9.2 \% \text { pred }) \\
\text { Cardiovascular disease: } n=14\end{array}$ & $\begin{array}{l}\text { All groups: } \\
\text { Pulmonary } \\
\text { rehabilitation } \\
50 \%-80 \% \text { of WRmax } \\
60 \% \text { of maximal } \\
\text { walking speed in the } \\
6 \mathrm{MWT} \\
50 \% \text { of } 1 \mathrm{RM}\end{array}$ & $\begin{array}{l}\text { All groups: } \\
6 \text { months } \\
2-3 \text { sessions/week } \\
2 \mathrm{~h} / \text { session }\end{array}$ & & \\
\hline \multirow{2}{*}{$\begin{array}{l}\text { Georgiopoulou et } \\
\text { al., } 2012 \\
\text { Greece }\end{array}$} & \multirow{2}{*}{ Pre-Post study } & \multirow{2}{*}{$\begin{array}{l}45 \text { patients with COPD }\left(40 \circ^{7} ;\right. \\
66.5 \pm 7.6 \text { years; } \mathrm{FEV}_{1} 45.7 \pm \\
18.7 \% \text { pred; FVC } 78.3 \pm \\
18.6 \% \text { pred })\end{array}$} & \multirow{2}{*}{$\begin{array}{l}\text { Pulmonary } \\
\text { rehabilitation } \\
60 \%-80 \% \text { of WRmax }\end{array}$} & \multirow{2}{*}{$\begin{array}{l}12 \text { weeks } \\
3 \text { sessions/week } \\
40 \mathrm{~min} / \mathrm{session}\end{array}$} & HR at rest (bpm) & $\begin{array}{l}\text { Pre } 88.0 \pm 10.7 \text { vs. Post } 83.3 \\
\pm 10.5, \mathrm{p}=0.004 \\
E S=-0.63\end{array}$ \\
\hline & & & & & HR recovery (bpm) & $\begin{array}{l}\text { Pre } 16.2 \pm 8.0 \text { vs. Post } 18.4 \\
\pm 8.4, \mathrm{p}=0.01 \\
E S=0.27\end{array}$ \\
\hline \multirow{3}{*}{$\begin{array}{l}\text { Lan et al., } 2013 \\
\text { Taiwan }\end{array}$} & \multirow{3}{*}{ Pre-Post study } & \multirow{3}{*}{$\begin{array}{l}26 \text { patients with COPD }(71.0 \pm \\
10.7 \text { years; } \mathrm{FEV}_{1} 64.8 \pm \\
23.0 \% \text { pred; FVC } 88.3 \pm \\
34.5 \% \text { pred })\end{array}$} & \multirow{3}{*}{$\begin{array}{l}\text { Pulmonary } \\
\text { rehabilitation } \\
75 \%-100 \% \text { of } \\
\text { VO2max }\end{array}$} & \multirow{3}{*}{$\begin{array}{l}12 \text { weeks } \\
2 \text { sessions/week } \\
40 \mathrm{~min} / \mathrm{session}\end{array}$} & HR (bpm) & $\begin{array}{l}\text { Pre } 134.5 \pm 14.9 \text { vs. Post } \\
137.4 \pm 19.9, \mathrm{p}=0.36 \\
E S=0.16\end{array}$ \\
\hline & & & & & $\begin{array}{l}\text { Mean blood pressure } \\
(\mathrm{mmHg})\end{array}$ & $\begin{array}{l}\text { Pre } 109.6 \pm 15.7 \text { vs. Post } \\
110.3 \pm 15.1, \mathrm{p}=0.72 \\
E S=0.05\end{array}$ \\
\hline & & & & & Oxygen pulse (ml/beat) & $\begin{array}{l}\text { Pre } 9.2 \pm 2.5 \text { vs. Post } 9.8 \pm \\
2.7, \mathrm{p}=0.02 \\
E S=0.23\end{array}$ \\
\hline \multirow{3}{*}{$\begin{array}{l}\text { Cheng et al., } 2014 \\
\text { Taiwan }\end{array}$} & \multirow{3}{*}{ Pre-Post study } & \multirow{3}{*}{$\begin{array}{l}64 \text { patients with COPD }\left(55 \sigma^{7} ;\right. \\
70.1 \pm 8.7 \text { years; } \mathrm{FEV}_{1} 44.9 \pm \\
11.7 \% \text { pred; FVC } 78.2 \pm \\
17.4 \% \text { pred })\end{array}$} & \multirow{3}{*}{$\begin{array}{l}\text { Pulmonary } \\
\text { rehabilitation } \\
60 \%-100 \% \text { of } \\
\text { VO2peak }\end{array}$} & \multirow{3}{*}{$\begin{array}{l}12 \text { weeks } \\
2 \text { sessions/week } \\
50 \mathrm{~min} / \mathrm{session}\end{array}$} & $\mathrm{HR}$ at rest (bpm) & $\begin{array}{l}\text { Pre } 87.2 \pm 12.7 \text { vs. Post } 83.9 \\
\pm 13.5, \mathrm{p}=0.048 \\
E S=-0.25\end{array}$ \\
\hline & & & & & Oxygen pulse (ml/beat) & $\begin{array}{l}\text { Pre } 7.2 \pm 1.9 \text { vs. Post } 7.9 \pm \\
2.2, \mathrm{p}=0.005 \\
E S=0.34\end{array}$ \\
\hline & & & & & Oxygen pulse (\%) & $\begin{array}{l}\text { Pre } 76.8 \pm 18.4 \text { vs. Post } 85.2 \\
\pm 24.8, p=0.003 \\
E S=0.38\end{array}$ \\
\hline
\end{tabular}


Table 2. Cont

\begin{tabular}{|c|c|c|c|c|c|c|}
\hline Study and Country & Study Design & Population & Intervention & $\begin{array}{l}\text { Duration and } \\
\text { Frequency }\end{array}$ & $\begin{array}{l}\text { Cardiovascular Outcomes } \\
\text { and Outcome Measures }\end{array}$ & $\begin{array}{c}\text { Results on Cardiovascular } \\
\text { Outcomes }\end{array}$ \\
\hline & & & & & Standard deviation of N-N & $\begin{array}{l}\text { At rest: Pre vs. Post, } p< \\
0.05 \\
\text { At exercise: Pre vs. Post, } \\
p<0.05\end{array}$ \\
\hline & & & & & $\begin{array}{l}\text { Square root of the mean } \\
\text { sum of the squares of the } \\
\text { difference between adjacent } \\
\text { normal R-R intervals }\end{array}$ & $\begin{array}{l}\text { At rest: Pre vs. Post, } \\
p<0.05 \\
\text { At exercise: Pre vs. Post, } \\
p<0.05\end{array}$ \\
\hline & & & & & Low frequency & $\begin{array}{l}\text { At rest: Pre vs. Post, } \\
p<0.05 \\
\text { At exercise: Pre vs. Post, } \\
p<0.05\end{array}$ \\
\hline & & & & & High frequency & $\begin{array}{l}\text { At rest: Pre vs. Post, } \\
p<0.05 \\
\text { At exercise: Pre vs. Post, } \\
p<0.05\end{array}$ \\
\hline & & & & & $\begin{array}{l}\text { Low frequency/High } \\
\text { frequency ratio }\end{array}$ & $\begin{array}{l}\text { At rest: Pre vs. Post, } \\
p<0.05 \\
\text { At exercise: Pre vs. Post, } \\
p<0.05\end{array}$ \\
\hline \multirow{5}{*}{$\begin{array}{l}\text { Borghi-Silva et al., } \\
2015 \\
\text { Brazil }\end{array}$} & \multirow{5}{*}{$\begin{array}{l}\text { Randomized controlled } \\
\text { trial }\end{array}$} & \multirow{5}{*}{$\begin{array}{l}10 \text { patients with COPD }\left(70^{7}\right. \\
67.0 \pm 7.0 \text { years; } \text { FEV }_{1} 32.0 \pm \\
11.0 \% \text { pred; FVC } 58.0 \pm \\
15.0 \% \text { pred })\end{array}$} & \multirow{5}{*}{$\begin{array}{l}\text { Aerobic training } \\
70 \% \text { of peak speed in } \\
\text { CPET }\end{array}$} & \multirow{5}{*}{$\begin{array}{l}12 \text { weeks } \\
3 \text { sessions/week } \\
30 \mathrm{~min} / \text { session }\end{array}$} & $\begin{array}{l}\text { Mean of RR and its } \\
\text { standard deviation at rest } \\
(\mathrm{ms})\end{array}$ & $\begin{array}{l}\text { Pre } 17.2 \pm 7.3 \text { vs. Post } 25.4 \\
\pm 5.5, p<0.05 \\
E S=1.27\end{array}$ \\
\hline & & & & & $\begin{array}{l}\text { Mean of RR and its } \\
\text { standard deviation at } \\
\text { constant speed (ms) }\end{array}$ & $\begin{array}{l}\text { Pre } 12.7 \pm 5.1 \text { vs. Post } 18.3 \\
\pm 4.7, p>0.05 \\
E S=1.14\end{array}$ \\
\hline & & & & & $\begin{array}{l}\text { Square root of the mean } \\
\text { squared differences of } \\
\text { successive RRi at rest (ms) }\end{array}$ & $\begin{array}{l}\text { Pre } 11.7 \pm 6.0 \text { vs. Post } 22.9 \\
\pm 0.2, p<0.05 \\
E S=2.64\end{array}$ \\
\hline & & & & & $\begin{array}{l}\text { Square root of the mean } \\
\text { squared differences of } \\
\text { successive RRi at constant } \\
\text { speed (ms) }\end{array}$ & $\begin{array}{l}\text { Pre } 3.5 \pm 1.7 \text { vs. Post } 16.9 \pm \\
7.0, p<0.05 \\
E S=2.63\end{array}$ \\
\hline & & & & & $\begin{array}{l}\text { Nonlinear indices - SD1 at } \\
\text { rest }\end{array}$ & $\begin{array}{l}\text { Pre } 7.1 \pm 4.2 \text { vs. Post } 19.2 \pm \\
11.8, p<0.05 \\
E S=1.37\end{array}$ \\
\hline
\end{tabular}


Table 2. Cont

\begin{tabular}{|c|c|c|c|c|c|c|}
\hline Study and Country & Study Design & Population & Intervention & $\begin{array}{l}\text { Duration and } \\
\text { Frequency }\end{array}$ & $\begin{array}{l}\text { Cardiovascular Outcomes } \\
\text { and Outcome Measures }\end{array}$ & $\begin{array}{c}\text { Results on Cardiovascular } \\
\text { Outcomes }\end{array}$ \\
\hline & & & & & $\begin{array}{l}\text { Nonlinear indices - SD1 at } \\
\text { constant speed }\end{array}$ & $\begin{array}{l}\text { Pre } 3.7 \pm 1.7 \text { vs. Post } 13.6 \pm \\
8.8, p<0.05 \\
E S=1.56\end{array}$ \\
\hline & & & & & $\begin{array}{l}\text { Nonlinear indices - SD2 at } \\
\text { rest }\end{array}$ & $\begin{array}{l}\text { Pre } 31.2 \pm 6.6 \text { vs. Post } 46.1 \\
\pm 22.0, p<0.05 \\
E S=0.92\end{array}$ \\
\hline & & & & & $\begin{array}{l}\text { Nonlinear indices - SD2 at } \\
\text { constant speed }\end{array}$ & $\begin{array}{l}\text { Pre } 17.3 \pm 5.9 \text { vs. Post } 25.4 \\
\pm 6.5, p<0.05 \\
E S=1.30\end{array}$ \\
\hline & & & & & Low frequency $(n u)$ & $\begin{array}{l}\text { Pre } 0.6 \pm 0.2 \text { vs. Post } 0.5 \pm \\
0.2, p>0.05 \\
E S=-0.60\end{array}$ \\
\hline & & & & & High frequency $(n u)$ & $\begin{array}{l}\text { Pre } 0.4 \pm 0.2 \text { vs. Post } 0.5 \pm \\
0.2, p>0.05 \\
E S=0.60\end{array}$ \\
\hline & & & & & $\begin{array}{l}\text { Low frequency/High } \\
\text { frequency ratio }\end{array}$ & $\begin{array}{l}\text { Pre } 2.4 \pm 2.3 \text { vs. Post } 1.8 \pm \\
1.7, p>0.05 \\
E S=-0.31\end{array}$ \\
\hline & & & & & Sample entropy & $\begin{array}{l}\text { Pre } 0.7 \pm 0.2 \text { vs. Post } 0.9 \pm \\
0.2, p>0.05 \\
E S=1.03\end{array}$ \\
\hline $\begin{array}{l}\text { Campos et al., } 2015 \\
\text { Chile }\end{array}$ & Pre-Post study & $\begin{array}{l}39 \text { patients with COPD }\left(36 \% 0^{7} ;\right. \\
67.3 \pm 8.5 \text { years; } \mathrm{FEV}_{1} 59.8 \pm \\
21.0 \% \text { pred; FVC } 78.0 \pm \\
20.3 \% \text { pred }) \\
\text { Arterial hypertension: } n=31 \\
\text { Dyslipidemia: } n=5 \\
\text { Congestive heart failure: } n=3\end{array}$ & $\begin{array}{l}\text { Pulmonary } \\
\text { rehabilitation } \\
70 \%-80 \% \text { of } 6 \mathrm{MWT}\end{array}$ & $\begin{array}{l}12 \text { weeks } \\
2 \text { sessions/week } \\
90 \mathrm{~min} / \text { session }\end{array}$ & & \\
\hline \multirow{3}{*}{$\begin{array}{l}\text { Leite et al., } 2015 \\
\text { Brazil }\end{array}$} & \multirow{3}{*}{$\begin{array}{l}\text { Non-randomized } \\
\text { controlled trial }\end{array}$} & \multirow{3}{*}{$\begin{array}{l}10 \text { patients with COPD }(62.0 \\
(60.3 ; 69.3) \text { years; FEV } 155.0 \\
(39.0 ; 70.0) \% \text { pred; FVC } 78.0 \\
(66.3 ; 83.5) \% \text { pred })\end{array}$} & \multirow{3}{*}{$\begin{array}{l}\text { Aerobic training } \\
60 \%-100 \% \text { of } \\
\text { VO2peak }\end{array}$} & \multirow{3}{*}{$\begin{array}{l}12 \text { weeks } \\
3 \text { sessions/week } \\
20-50 \mathrm{~min} / \text { session }\end{array}$} & $\begin{array}{l}\text { Standard deviation of the } \\
\text { mean of all normal RR } \\
\text { intervals (ms) }\end{array}$ & $\begin{array}{l}\text { Pre } 19.8 \pm 6.2 \text { vs. Post } 24.9 \\
\pm 8.6, p>0.05 \\
E S=0.67\end{array}$ \\
\hline & & & & & $\begin{array}{l}\text { Root mean square of } \\
\text { differences between } \\
\text { adjacent normal RR } \\
\text { intervals in a time interval } \\
(\mathrm{ms})\end{array}$ & $\begin{array}{l}\text { Pre } 14.2 \pm 5.7 \text { vs. Post } 18.3 \\
\pm 6.2, p>0.05 \\
E S=0.69\end{array}$ \\
\hline & & & & & $\begin{array}{l}\text { Spectral component of low } \\
\text { frequency (ms2) }\end{array}$ & $\begin{array}{l}\text { Pre } 146.1 \pm 118.9 \text { vs. Post } \\
177.7 \pm 125.6, p>0.05 \\
E S=0.26\end{array}$ \\
\hline
\end{tabular}


Table 2. Cont.

\begin{tabular}{|c|c|c|c|c|c|c|}
\hline \multirow[t]{5}{*}{ Study and Country } & \multirow[t]{5}{*}{ Study Design } & \multirow[t]{5}{*}{ Population } & \multirow[t]{5}{*}{ Intervention } & \multirow[t]{5}{*}{$\begin{array}{c}\text { Duration and } \\
\text { Frequency }\end{array}$} & \multirow{2}{*}{$\begin{array}{l}\text { Cardiovascular Outcomes } \\
\text { and Outcome Measures } \\
\begin{array}{l}\text { Spectral component of low } \\
\text { frequency }(\mathrm{nu})\end{array}\end{array}$} & \multirow{2}{*}{$\begin{array}{l}\begin{array}{c}\text { Results on Cardiovascular } \\
\text { Outcomes }\end{array} \\
\text { Pre } 67.5 \pm 16.0 \text { vs. Post } 58.5 \\
\pm 13.6, p>0.05 \\
E S=-0.61\end{array}$} \\
\hline & & & & & & \\
\hline & & & & & $\begin{array}{l}\text { Spectral component of high } \\
\text { frequency (ms2) }\end{array}$ & $\begin{array}{l}\text { Pre } 62.3 \pm 46.8 \text { vs. Post } \\
113.2 \pm 62.2, p<0.05 \\
E S=0.92\end{array}$ \\
\hline & & & & & $\begin{array}{l}\text { Spectral component of high } \\
\text { frequency }(\mathrm{nu})\end{array}$ & $\begin{array}{l}\text { Pre } 32.6 \pm 15.9 \text { vs. Post } 41.5 \\
\pm 13.6, p>0.05 \\
E S=0.60\end{array}$ \\
\hline & & & & & $\begin{array}{l}\text { Low frequency/High } \\
\text { frequency ratio }\end{array}$ & $\begin{array}{l}\text { Pre } 2.9 \pm 2.2 \text { vs. Post } 1.6 \pm \\
0.8, p>0.05 \\
E S=-0.78\end{array}$ \\
\hline $\begin{array}{l}\text { Mkacher et al., } 2015 \\
\text { Tunisia }\end{array}$ & $\begin{array}{l}\text { Randomized controlled } \\
\text { trial }\end{array}$ & $\begin{array}{l}68 \text { patients with COPD } \\
\text { Pulmonary rehabilitation } \\
\text { group: } 33 \text { ( } 33 \sigma^{7} ; \\
61.2 \pm 3.2 \text { years; } \mathrm{FEV}_{1} 38.6 \pm \\
8.6 \% \text { pred) } \\
\text { Pulmonary } \\
\text { rehabilitation+balance group: } \\
35\left(350^{7} \text {; }\right. \\
58.3 \pm 4.3 \text { years; } \mathrm{FEV}_{1} 39.4 \pm \\
10.3 \% \text { pred) }\end{array}$ & $\begin{array}{l}\text { All groups: } \\
\text { Pulmonary } \\
\text { rehabilitation }\end{array}$ & $\begin{array}{l}\text { All groups: } \\
6 \text { months } \\
6 \text { sessions/week (3 } \\
\text { times/week, } 2 \\
\text { sessions/day) }\end{array}$ & HR at rest (bpm) & $\begin{array}{l}\text { Pulmonary rehabilitation } \\
\text { group: Pre } 72.7 \pm 8.9 \text { vs. } \\
\text { Post } 73.0 \pm 4.3, p>0.05 \\
E S=0.04 \\
\text { Pulmonary } \\
\text { rehabilitation+balance } \\
\text { group: Pre } 75.3 \pm 3.9 \text { vs. } \\
\text { Post } 73.5 \pm 4.5, p>0.05 \\
E S=-0.43\end{array}$ \\
\hline $\begin{array}{l}\text { Spielmanns et al., } \\
2015 \\
\text { Germany }\end{array}$ & $\begin{array}{l}\text { Randomized controlled } \\
\text { trial }\end{array}$ & 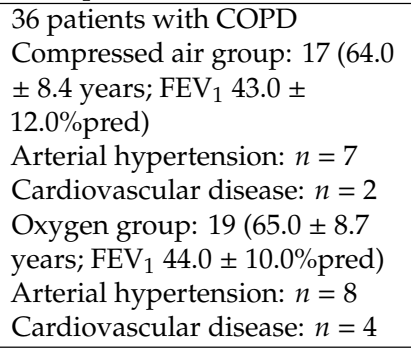 & $\begin{array}{l}\text { All groups: } \\
\text { Continuous aerobic } \\
\text { training } \\
70 \%-85 \% \text { of WRmax } \\
\text { Interval aerobic } \\
\text { training } \\
110 \%-125 \% \text { of } \\
\text { WRmax }\end{array}$ & $\begin{array}{l}\text { All groups: } \\
24 \text { weeks } \\
3 \text { sessions/week } \\
30 \text { min/session }\end{array}$ & & \\
\hline $\begin{array}{l}\text { Cardoso et al., } 2016 \\
\text { Brazil }\end{array}$ & $\begin{array}{l}\text { Non-randomized } \\
\text { controlled trial }\end{array}$ & $\begin{array}{l}10 \text { patients with COPD }(65.2 \pm \\
4.2 \text { years; FEV } 141.8 \pm \\
21.3 \% \text { pred; FVC } 60.7 \pm \\
18.0 \% \text { pred) } \\
\text { Arterial hypertension: } n=7\end{array}$ & $\begin{array}{l}\text { Pulmonary } \\
\text { rehabilitation } \\
75 \% \text { of WRmax } \\
60 \% \text { of } 1 \mathrm{RM}\end{array}$ & $\begin{array}{l}12 \text { weeks } \\
3 \text { sessions/week } \\
>30 \mathrm{~min} / \mathrm{session}\end{array}$ & & \\
\hline
\end{tabular}


Table 2. Cont.

\begin{tabular}{|c|c|c|c|c|c|c|}
\hline Study and Country & Study Design & Population & Intervention & $\begin{array}{l}\text { Duration and } \\
\text { Frequency }\end{array}$ & $\begin{array}{l}\text { Cardiovascular Outcomes } \\
\text { and Outcome Measures }\end{array}$ & $\begin{array}{c}\text { Results on Cardiovascular } \\
\text { Outcomes }\end{array}$ \\
\hline \multirow{2}{*}{$\begin{array}{l}\text { Engel et al., } 2016 \\
\text { Australia }\end{array}$} & \multirow{2}{*}{$\begin{array}{l}\text { Randomized controlled } \\
\text { trial }\end{array}$} & \multirow{2}{*}{$\begin{array}{l}33 \text { patients with COPD }\left(10 \sigma^{7} ;\right. \\
65.5 \pm 4.0 \text { years; FEV } 1.6 \pm 0.5 \\
\text { L; FVC } 2.3 \pm 0.7 \mathrm{~L})\end{array}$} & \multirow{2}{*}{$\begin{array}{l}\text { Pulmonary } \\
\text { rehabilitation }\end{array}$} & \multirow{2}{*}{16 weeks } & $\begin{array}{l}\text { Systolic blood pressure } \\
(\mathrm{mmHg})\end{array}$ & $\begin{array}{l}\text { Mean Pre/Post difference } \\
\text { Group 1: }-3.6,95 \% \mathrm{CI} \\
(-13.5 ; 6.3) \\
\text { Group 2: }-10.6,95 \% \mathrm{CI} \\
(-19.6 ;-1.5) \\
\text { Group 3: }-8.3,95 \% \mathrm{CI} \\
(-20.5 ; 3.8)\end{array}$ \\
\hline & & & & & $\begin{array}{l}\text { Diastolic blood pressure } \\
(\mathrm{mmHg})\end{array}$ & $\begin{array}{l}\text { Mean Pre/Post difference } \\
\text { Group 1: }-3.5,95 \% \mathrm{CI} \\
(-12.6 ; 5.6) \\
\text { Group 2: }-7.7,95 \% \mathrm{CI} \\
(-17.1 ; 1.8) \\
\text { Group 3: }-4.7,95 \% \mathrm{CI} \\
(-13.5 ; 4.2)\end{array}$ \\
\hline $\begin{array}{l}\text { Boeselt et al., } 2017 \\
\text { Germany }\end{array}$ & $\begin{array}{l}\text { Non-randomized } \\
\text { controlled trial }\end{array}$ & $\begin{array}{l}20 \text { patients with COPD }\left(160^{7} ;\right. \\
65.9 \pm 8.2 \text { years; } \mathrm{FEV}_{1} 67.9 \pm \\
29.2 \% \text { pred }) \\
\text { Arterial hypertension: } n=5 \\
\text { Cardiovascular disease: } n=2\end{array}$ & $\begin{array}{l}\text { Strength training } \\
35 \%-75 \% \text { of } 1 \text { RM }\end{array}$ & $\begin{array}{l}3 \text { months } \\
2 \text { sessions/week } \\
90 \mathrm{~min} / \text { session }\end{array}$ & & \\
\hline $\begin{array}{l}\text { Kanao et al., } 2017 \\
\text { Japan }\end{array}$ & Pre-Post study & $\begin{array}{l}29 \text { patients with COPD }\left(260^{7} ;\right. \\
73.2 \pm 5 \text { years; } \mathrm{FEV}_{1} 51.0 \pm \\
121.3 \% \text { pred }) \\
\text { Arterial hypertension: } n=10 \\
\text { Cardiovascular disease: } n=5\end{array}$ & $\begin{array}{l}\text { Pulmonary } \\
\text { rehabilitation } \\
60 \% \text { of WRpeak }\end{array}$ & $\begin{array}{l}12 \text { weeks } \\
2 \text { sessions/week }\end{array}$ & & \\
\hline $\begin{array}{l}\text { Pacheco et al., } 2017 \\
\text { Spain }\end{array}$ & Observational study & $\begin{array}{l}35 \text { patients with COPD } \\
\left(88.6 \% \sigma^{7} ; 65.1 \pm 9.0 \text { years; } \mathrm{FEV}_{1}\right. \\
42.2 \pm 10.5 ; \mathrm{FVC} 67.8 \pm \\
13.3 \% \text { pred; DLCO } 47.9 \pm \\
21.0 \% \text { pred } \\
\text { Arterial hypertension: } n=20 \\
\text { Dyslipidemia: } n=6 \\
\text { Congestive heart failure: } n=7 \\
\text { Ischemic cardiomyopathy: } \\
n=3\end{array}$ & $\begin{array}{l}\text { Pulmonary } \\
\text { rehabilitation } \\
70 \% \text { of } \mathrm{WRmax} \\
75 \% \text { of } 1 \mathrm{RM}\end{array}$ & $\begin{array}{l}12 \text { weeks } \\
3 \text { sessions/week } \\
>30 \mathrm{~min} / \text { session }\end{array}$ & & \\
\hline
\end{tabular}


Table 2. Cont

\begin{tabular}{|c|c|c|c|c|c|c|}
\hline Study and Country & Study Design & Population & Intervention & $\begin{array}{l}\text { Duration and } \\
\text { Frequency }\end{array}$ & $\begin{array}{l}\text { Cardiovascular Outcomes } \\
\text { and Outcome Measures }\end{array}$ & $\begin{array}{c}\text { Results on Cardiovascular } \\
\text { Outcomes }\end{array}$ \\
\hline \multirow{5}{*}{$\begin{array}{l}\text { Papp et al., } 2017 \\
\text { Sweden }\end{array}$} & \multirow{5}{*}{$\begin{array}{l}\text { Randomized controlled } \\
\text { trial }\end{array}$} & \multirow{5}{*}{$\begin{array}{l}17 \text { patients with } \mathrm{COPD}\left(7 \sigma^{7} ;\right. \\
69.0(62.0 ; 72.1) \text { years; } \mathrm{FEV}_{1} \\
64.3 \pm 15.4 \% \text { pred })\end{array}$} & \multirow{5}{*}{$\begin{array}{l}\text { Aerobic and strength } \\
\text { training } \\
70 \% \text { of } 1 \text { RM } \\
\text { Perceived exertion } \\
12-14 \text { in the Borg }\end{array}$} & \multirow{5}{*}{$\begin{array}{l}12 \text { weeks } \\
2 \text { sessions/week } \\
60-70 \mathrm{~min} / \text { session }\end{array}$} & HR at rest $(\mathrm{bpm})$ & $\begin{array}{l}\text { Mean Pre/Post difference } \\
0.6, p=0.82\end{array}$ \\
\hline & & & & & $\begin{array}{l}\text { Systolic blood pressure at } \\
\text { rest }(\mathrm{mmHg})\end{array}$ & $\begin{array}{l}\text { Mean Pre/Post difference } \\
4.2\end{array}$ \\
\hline & & & & & $\begin{array}{l}\text { Diastolic blood pressure at } \\
\text { rest (mmHg) }\end{array}$ & $\begin{array}{l}\text { Mean Pre/Post difference } \\
5.7, p=0.04\end{array}$ \\
\hline & & & & & $\begin{array}{l}\text { Number of pairs of adjacent } \\
\text { NN intervals differing by } \\
\text { more than } 50 \text { ms in the } 5 \\
\text { min recording divided by } \\
\text { the total number of all NN } \\
\text { intervals }(\%)\end{array}$ & $\begin{array}{l}\text { Mean Pre/Post difference } \\
0.6, p=0.56\end{array}$ \\
\hline & & & & & $\begin{array}{l}\text { Square root of the mean of } \\
\text { the sum of the squares of } \\
\text { differences between } \\
\text { adjacent NN intervals }\end{array}$ & $\begin{array}{l}\text { Mean Pre/Post difference } \\
-3.2, p=0.27\end{array}$ \\
\hline $\begin{array}{l}\text { Vasilopoulou et al., } \\
2017 \\
\text { Greece }\end{array}$ & $\begin{array}{l}\text { Randomized controlled } \\
\text { trial }\end{array}$ & $\begin{array}{l}50 \text { patients with COPD }\left(380^{7} ;\right. \\
66.7 \pm 7.3 \text { years; } \mathrm{FEV}_{1} 51.8 \pm \\
17.3 \% \text { pred; FVC } 78.4 \pm \\
18.4 \% \text { pred; DLCO } 57.0 \pm \\
20.4 \% \text { pred) } \\
\text { Cardiovascular disease: } n=15\end{array}$ & $\begin{array}{l}\text { Pulmonary } \\
\text { rehabilitation }\end{array}$ & $\begin{array}{l}12 \text { months } \\
2 \text { sessions/week }\end{array}$ & & \\
\hline \multirow{3}{*}{$\begin{array}{l}\text { Lan et al., } 2018 \\
\text { Taiwan }\end{array}$} & \multirow{3}{*}{ Pre-Post study } & \multirow{3}{*}{$\begin{array}{l}43 \text { patients with COPD }\left(310^{7} ;\right. \\
69.7 \pm 8.8 \text { years; } \mathrm{FEV}_{1} 49.5 \pm \\
19.9 \% \text { pred; FVC } 76.5 \pm \\
22.3 \% \text { pred })\end{array}$} & \multirow{3}{*}{$\begin{array}{l}\text { Pulmonary } \\
\text { rehabilitation }\end{array}$} & \multirow{3}{*}{$\begin{array}{l}12 \text { weeks } \\
2 \text { sessions/week } \\
40 \mathrm{~min} / \mathrm{session}\end{array}$} & HR at rest & Pre vs. Post $p>0.05$ \\
\hline & & & & & Mean blood pressure at rest & Pre vs. Post $p<0.05$ \\
\hline & & & & & Oxygen pulse & Pre vs. Post $p<0.05$ \\
\hline $\begin{array}{l}\text { Moezy et al., } 2018 \\
\text { Iran }\end{array}$ & $\begin{array}{l}\text { Randomized controlled } \\
\text { trial }\end{array}$ & $\begin{array}{l}14 \text { patients with COPD } \\
\left(71.4 \% \sigma^{7} \text {; }\right. \\
64.7 \pm 7.5 \text { years; } \mathrm{FEV}_{1} 60.2 \pm \\
14.0 \% \text { pred })\end{array}$ & $\begin{array}{l}\text { Aerobic training } \\
\text { Dyspnoea 3-4 in the } \\
\text { mBorg }\end{array}$ & $\begin{array}{l}12 \text { weeks } \\
3 \text { sessions/week } \\
15-60 \mathrm{~min} / \text { session }\end{array}$ & HR at rest (bpm) & $\begin{array}{l}\text { Pre } 80.4 \pm 12.6 \text { vs. Post } 77.8 \\
\pm 11.9, \mathrm{p}=0.968 \\
E S=-0.21\end{array}$ \\
\hline
\end{tabular}


Table 2. Cont.

\begin{tabular}{|c|c|c|c|c|c|c|}
\hline Study and Country & Study Design & Population & Intervention & $\begin{array}{l}\text { Duration and } \\
\text { Frequency }\end{array}$ & $\begin{array}{l}\text { Cardiovascular Outcomes } \\
\text { and Outcome Measures }\end{array}$ & $\begin{array}{c}\text { Results on Cardiovascular } \\
\text { Outcomes }\end{array}$ \\
\hline \multirow{4}{*}{$\begin{array}{l}\text { Silva et al., } 2018 \\
\text { Brazil }\end{array}$} & \multirow{4}{*}{$\begin{array}{l}\text { Randomized controlled } \\
\text { trial }\end{array}$} & \multirow{4}{*}{$\begin{array}{l}48 \text { patients with COPD } \\
\text { Elastic resistances group: } 32 \\
(69.4 \pm 9.0 \text { years; FEV } 150.7 \pm \\
16.7 \% \text { pred; FVC } 72.5 \pm \\
13.2 \% \text { pred }) \\
\text { Weight machines group: } 16 \\
(64.9 \pm 11.2 \text { years; FEV } 16.4 \pm \\
15.2 \% \text { pred; FVC } 66.1 \pm \\
14.0 \% \text { pred) }\end{array}$} & \multirow{4}{*}{$\begin{array}{l}\text { All groups: } \\
\text { Strength training }\end{array}$} & \multirow{4}{*}{$\begin{array}{l}\text { All groups: } \\
12 \text { weeks } \\
3 \text { sessions/week } \\
60 \mathrm{~min} / \mathrm{session}\end{array}$} & Total cholesterol (mg/dL) & $\begin{array}{l}\text { Elastic resistances group: } \\
\text { Pre } 108.4 \pm 25.3 \text { vs. Post } \\
104.6 \pm 14.3, p>0.05 \\
E S=-0.18 \\
\text { Weight machines group: } \\
\text { Pre } 84.6 \pm 27.0 \text { vs. Post } 71.1 \\
\pm 32.0, p>0.05 \\
E S=-0.46\end{array}$ \\
\hline & & & & & Cholesterol - HDL (mg/dL) & $\begin{array}{l}\text { Elastic resistances group: } \\
\text { Pre } 58.4 \pm 23.2 \text { vs. Post } 63.4 \\
\pm 17.3, p>0.05 \\
E S=0.24 \\
\text { Weight machines group: } \\
\text { Pre } 132.3 \pm 43.6 \text { vs. Post } \\
150.3 \pm 52.3, p>0.05 \\
E S=0.37\end{array}$ \\
\hline & & & & & $\begin{array}{l}\text { Cholesterol - Triglycerides } \\
(\mathrm{mg} / \mathrm{dL})\end{array}$ & $\begin{array}{l}\text { Elastic resistances group: } \\
\text { Pre } 154.2 \pm 62.3 \text { vs. Post } \\
129.7 \pm 40.3, p>0.05 \\
E S=-0.47 \\
\text { Weight machines group: } \\
\text { Pre } 104.8 \pm 38.4 \text { vs. Post } \\
99.9 \pm 32.9, p>0.05 \\
E S=-0.14\end{array}$ \\
\hline & & & & & $\begin{array}{l}\text { Total cholesterol/HDL ratio } \\
(\mathrm{mg} / \mathrm{dL})\end{array}$ & $\begin{array}{l}\text { Elastic resistances group: } \\
\text { Pre } 50.7 \pm 39.9 \text { vs. Post } 40.9 \\
\pm 25.8, p>0.05 \\
E S=-0.29 \\
\text { Weight machines group: } \\
\text { Pre } 71.9 \pm 31.2 \text { vs. Post } 61.3 \\
\pm 15.4, p>0.05 \\
E S=-0.43\end{array}$ \\
\hline $\begin{array}{l}\text { Charikiopoulou et } \\
\text { al., } 2019 \\
\text { Greece }\end{array}$ & Non-controlled study & $\begin{array}{l}32 \text { patients with COPD }\left(250^{7} ;\right. \\
66.0 \pm 6.0 \text { years; } \mathrm{FEV}_{1} 43.1 \pm \\
15.1 \% \text { pred; DLCO } 38.2 \pm \\
22.8 \% \text { pred }) \\
\text { Cardiovascular disease: } n=22\end{array}$ & $\begin{array}{l}\text { Pulmonary } \\
\text { rehabilitation } \\
100 \% \text { of WRmax }\end{array}$ & $\begin{array}{l}13 \text { weeks } \\
2 \text { sessions/week } \\
\geq 1 \mathrm{~h} / \text { session }\end{array}$ & & \\
\hline
\end{tabular}


Table 2. Cont.

\begin{tabular}{|c|c|c|c|c|c|c|}
\hline Study and Country & Study Design & Population & Intervention & $\begin{array}{l}\text { Duration and } \\
\text { Frequency }\end{array}$ & $\begin{array}{l}\text { Cardiovascular Outcomes } \\
\text { and Outcome Measures }\end{array}$ & $\begin{array}{c}\text { Results on Cardiovascular } \\
\text { Outcomes }\end{array}$ \\
\hline $\begin{array}{l}\text { Mekki et al., } 2019 \\
\text { Tunisia }\end{array}$ & $\begin{array}{l}\text { Randomized controlled } \\
\text { trial }\end{array}$ & 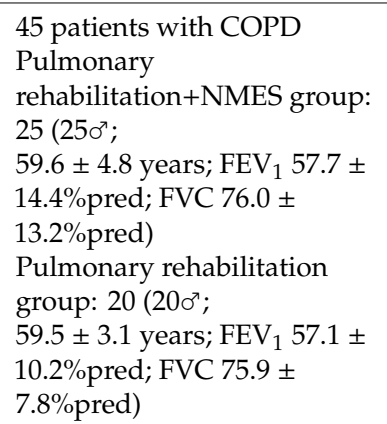 & $\begin{array}{l}\text { All groups: } \\
\text { Pulmonary } \\
\text { rehabilitation } \\
60 \%-70 \% \text { of HRmax } \\
\text { in the } 6 \mathrm{MWT} \\
50 \%-85 \% \text { of } 10 \mathrm{RM}\end{array}$ & $\begin{array}{l}\text { All groups: } \\
6 \text { months } \\
3 \text { sessions/week } \\
80 \text { min/session }\end{array}$ & $\mathrm{HR}$ at rest (bpm) & $\begin{array}{l}\text { Pulmonary } \\
\text { rehabilitation+NMES } \\
\text { group: Pre } 80.0 \pm 9.0 \text { vs. } \\
\text { Post } 78.0 \pm 9.0, p<0.001 \\
E S=-0.22 \\
\text { Pulmonary rehabilitation } \\
\text { group: Pre } 80.0 \pm 7.0 \text { vs. } \\
\text { Post } 77.0 \pm 7.0, p<0.001 \\
E S=-0.43\end{array}$ \\
\hline \multirow{3}{*}{$\begin{array}{l}\text { Silva et al., } 2019 \\
\text { Brazil }\end{array}$} & \multirow{3}{*}{$\begin{array}{l}\text { Randomized controlled } \\
\text { trial }\end{array}$} & \multirow{3}{*}{$\begin{array}{l}19 \text { patients with COPD } \\
\text { Elastic resistances group: } 9 \\
(65.9 \pm 8.9 \text { years; FEV } 145.2 \pm \\
16.2 \% \text { pred; FVC } 64.7 \pm \\
19.0 \% \text { pred }) \\
\text { Weight machines group: } 10 \\
(65.5 \pm 9.8 \text { years; FEV } 157.6 \pm \\
16.3 \% \text { pred; FVC } 79.8 \pm \\
11.5 \% \text { pred })\end{array}$} & \multirow{3}{*}{$\begin{array}{l}\text { All groups: } \\
\text { Strength training }\end{array}$} & \multirow{3}{*}{$\begin{array}{l}\text { All groups: } \\
12 \text { weeks } \\
3 \text { sessions/week } \\
60 \mathrm{~min} / \mathrm{session}\end{array}$} & HR (bpm) & $\begin{array}{l}\text { Elastic resistances group: } \\
\text { Pre } 74.1 \pm 8.8 \text { vs. Post } 76.8 \\
\pm 8.9, p>0.05 \\
E S=0.30 \\
\text { Weight machines group: } \\
\text { Pre } 71.4 \pm 6.4 \text { vs. Post } 68.9 \\
\pm 9.9, p>0.05 \\
E S=-0.30\end{array}$ \\
\hline & & & & & $\begin{array}{l}\text { Systolic blood pressure } \\
(\mathrm{mmHg})\end{array}$ & $\begin{array}{l}\text { Elastic resistances group: } \\
\text { Pre } 120.0(105.0 ; 135.0) \text { vs. } \\
\text { Post } 120.0(110.0 ; 120.0), \\
p>0.05 \\
\text { Weight machines group: } \\
\text { Pre } 120.0(117.5 ; 130.0) \text { vs. } \\
\text { Post } 120.0(110.0 ; 120.0), \\
p>0.05\end{array}$ \\
\hline & & & & & $\begin{array}{l}\text { Diastolic blood pressure } \\
(\mathrm{mmHg})\end{array}$ & $\begin{array}{l}\text { Elastic resistances group: } \\
\text { Pre } 70.0(70.0 ; 80.0) \text { vs. Post } \\
70.0(70.0 ; 75.0), p>0.05 \\
\text { Weight machines group: } \\
\text { Pre } 80.0(70.0 ; 90.0) \text { vs. Post } \\
75.0(67.5 ; 80.0), p>0.05\end{array}$ \\
\hline
\end{tabular}

Data are presented as mean \pm standard deviation or median (interquartile range), unless otherwise stated. Legend: 6MWT, 6-min walk test; 12MWT, 12-min walk test; 1RM, one repetition maximum; 10RM, ten repetition maximum; 15RM, fifteen repetition maximum; $95 \% \mathrm{CI}$, 95\% confidence interval; \%pred, percentage predicted; COPD, chronic obstructive pulmonary disease; CPET, cardiopulmonary exercise test; DLCO, diffusing capacity for carbon monoxide; ES, effect size; FEV 1 , forced expiratory volume in $1 \mathrm{~s}$; FVC, forced vital capacity; HDL, high density lipoprotein; $\mathrm{HR}$, heart rate; $\mathrm{HR}_{\max }$, maximum heart rate; ILD, interstitial lung disease; IPF, idiopathic pulmonary fibrosis; LDL, low density lipoprotein; mBorg, modified Borg scale; NMES, neuromuscular electrical stimulation; $\mathrm{VO}_{2} \mathrm{max}$, maximal oxygen uptake; $\mathrm{VO}_{2}$ peak, peak oxygen uptake; $\mathrm{WR}$, work rate; $\mathrm{WR}_{\max }$, maximal work rate; $\mathrm{WR}$ peak, peak work rate. 
Table 3. Characteristics of the studies in patients with asthma included in phase 2 (i.e., studies that specified the prevalence of cardiovascular comorbidities in the baseline characteristics of the population under study and/or reported at least one cardiovascular outcome) $(n=2)$.

\begin{tabular}{|c|c|c|c|c|c|c|}
\hline Study and Country & Study design & Population & Intervention & $\begin{array}{l}\text { Duration and } \\
\text { Frequency }\end{array}$ & $\begin{array}{c}\text { Cardiovascular } \\
\text { Outcomes and } \\
\text { Outcome measures }\end{array}$ & $\begin{array}{l}\text { Results on } \\
\text { Cardiovascular } \\
\text { Outcomes }\end{array}$ \\
\hline \multirow{4}{*}{$\begin{array}{l}\text { Cochrane et al., } 1990 \\
\text { Scotland }\end{array}$} & \multirow{4}{*}{$\begin{array}{l}\text { Randomized } \\
\text { controlled trial }\end{array}$} & \multirow{4}{*}{$\begin{array}{c}18 \text { patients with } \\
\text { Asthma }(27.0 \pm 17.0 \\
\text { years; } \mathrm{FEV}_{1} 76.0 \pm \\
12.0 \% \text { pred })\end{array}$} & \multirow{4}{*}{$\begin{array}{l}\text { Aerobic and muscle } \\
\text { strength training } \\
75 \% \text { of HRmax }\end{array}$} & \multirow{4}{*}{$\begin{array}{c}3 \text { months } \\
3 \text { sessions/week } \\
30 \mathrm{~min} / \text { session }\end{array}$} & $\begin{array}{l}\text { Oxygen pulse } \\
\text { (mL/beat) }\end{array}$ & $\begin{array}{l}\text { Pre } 8.8 \pm 2.3 \text { vs. Post } \\
10.8 \pm 2.4, p<0.001 \\
E S=0.85\end{array}$ \\
\hline & & & & & $\begin{array}{l}\text { Total blood } \\
\text { cholesterol }(\mathrm{mmol} / \mathrm{L})\end{array}$ & $\begin{array}{l}\text { Pre } 5.4 \pm 1.1 \text { vs. Post } \\
5.3 \pm 1.1, p>0.05 \\
E S=-0.09\end{array}$ \\
\hline & & & & & $\begin{array}{l}\text { Cholesterol - HDL } \\
(\mathrm{mmol} / \mathrm{L})\end{array}$ & $\begin{array}{l}\text { Pre } 1.7 \pm 0.4 \text { vs. Post } \\
1.6 \pm 0.3, p>0.05 \\
E S=-0.28\end{array}$ \\
\hline & & & & & $\begin{array}{l}\text { Cholesterol - LDL } \\
(\mathrm{mmol} / \mathrm{L})\end{array}$ & $\begin{array}{l}\text { Pre } 3.2 \pm 1.2 \text { vs. Post } \\
2.9 \pm 0.9, p>0.05 \\
E S=-0.28\end{array}$ \\
\hline \multirow{3}{*}{$\begin{array}{l}\text { El-Kader et al., } 2016 \\
\text { Saudi Arabia }\end{array}$} & \multirow{3}{*}{$\begin{array}{l}\text { Randomized } \\
\text { controlled trial }\end{array}$} & \multirow{3}{*}{$\begin{array}{c}40 \text { patients with } \\
\text { Asthma }\left(23 \sigma^{7} ; 47.2 \pm\right. \\
6.5 \text { years; } \mathrm{FEV}_{1} 1.4 \pm \\
0.7 \mathrm{~L})\end{array}$} & \multirow{3}{*}{$\begin{array}{l}\text { Aerobic training } \\
60 \%-80 \% \text { of HRmax }\end{array}$} & \multirow{3}{*}{$\begin{array}{c}6 \text { months } \\
3 \text { sessions/week } \\
30 \mathrm{~min} / \text { session }\end{array}$} & $\begin{array}{l}\text { Cholesterol - HDL } \\
(\mathrm{mg} / \mathrm{dL})\end{array}$ & $\begin{array}{l}\text { Pre } 34.7 \pm 5.6 \text { vs. Post } \\
37.9 \pm 4.6, p<0.05 \\
E S=0.62\end{array}$ \\
\hline & & & & & $\begin{array}{l}\text { Cholesterol - LDL } \\
(\mathrm{mg} / \mathrm{dL})\end{array}$ & $\begin{array}{l}\text { Pre } 133.7 \pm 13.2 \text { vs. } \\
\text { Post } 120.3 \pm 11.5 \\
p<0.05 \\
E S=-1.08\end{array}$ \\
\hline & & & & & $\begin{array}{l}\text { Cholesterol - } \\
\text { Triglycerides (mg/dL) }\end{array}$ & $\begin{array}{l}\text { Pre } 155.4 \pm 12.6 \text { vs. } \\
\text { Post } 127.7 \pm 11.3 \\
p<0.05 \\
E S=-2.31\end{array}$ \\
\hline
\end{tabular}

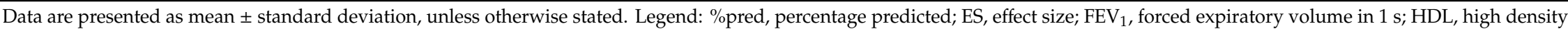
lipoprotein; $\mathrm{HR}$, heart rate; $\mathrm{HR}_{\max }$, maximum heart rate; $\mathrm{LDL}$, low density lipoprotein 
Table 4. Characteristics of the studies in patients with ILD included in phase 2 (i.e., studies that specified the prevalence of cardiovascular comorbidities in the baseline characteristics of the population under study and/or reported at least one cardiovascular outcome) $(n=8)$.

\begin{tabular}{|c|c|c|c|c|c|c|}
\hline Study and Country & Study Design & Population & Intervention & $\begin{array}{c}\text { Duration and } \\
\text { Frequency }\end{array}$ & $\begin{array}{c}\text { Cardiovascular Outcomes } \\
\text { and Outcome Measures }\end{array}$ & $\begin{array}{c}\text { Results on Cardiovascular } \\
\text { Outcomes }\end{array}$ \\
\hline $\begin{array}{l}\text { Gaunaurd et al., } \\
2014 \\
\text { United States of } \\
\text { America }\end{array}$ & $\begin{array}{l}\text { Randomized controlled } \\
\text { trial }\end{array}$ & $\begin{array}{l}11 \text { patients with IPF }(71.0 \pm 6.0 \\
\text { years; FVC } 60.0 \pm 11.0 \% \text { pred; } \\
\text { DLCO } 44.0 \pm 11.0 \% \text { pred }) \\
\text { History of heart disease: } n=1\end{array}$ & $\begin{array}{l}\text { Pulmonary } \\
\text { rehabilitation } \\
70 \%-80 \% \text { of HRmax }\end{array}$ & $\begin{array}{l}12 \text { weeks } \\
2 \text { sessions/week } \\
90 \mathrm{~min} / \text { session }\end{array}$ & & \\
\hline $\begin{array}{l}\text { Vainshelboim et al., } \\
2014 \\
\text { Israel }\end{array}$ & $\begin{array}{l}\text { Randomized controlled } \\
\text { trial }\end{array}$ & $\begin{array}{l}15 \text { patients with IPF }\left(100^{\top} ;\right. \\
68.8 \pm 6 \text { years; FEV } 68.5 \pm \\
15.8 \% \text { pred; FVC } 66.1 \pm \\
14.8 \% \text { pred; DLCO } 48.6 \pm \\
17.2 \% \text { pred) } \\
\text { Arterial hypertension: } n=12 \\
\text { Coronary heart disease: } n=7 \\
\text { Pulmonary hypertension: } n=5\end{array}$ & $\begin{array}{l}\text { Aerobic and strength } \\
\text { training } \\
50 \%-70 \% \text { of WRpeak } \\
70 \%-90 \% \text { of average } \\
\text { walking speed in the } \\
6 \mathrm{MWT} \\
3-6 \text { in the mBorg }\end{array}$ & $\begin{array}{l}12 \text { weeks } \\
2 \text { sessions/week } \\
60 \mathrm{~min} / \text { session }\end{array}$ & $\begin{array}{l}\text { HR at rest (bpm) } \\
\text { Systolic blood pressure at } \\
\text { rest (mmHg) } \\
\text { Diastolic blood pressure at } \\
\text { rest (mmHg) } \\
\text { Oxygen pulse (ml/beat) }\end{array}$ & $\begin{array}{l}\text { Mean Pre/Post difference } \\
-2.4 \pm 9.1 \\
E S=-0.26 \\
\text { Mean Pre/Post difference } \\
-2.9 \pm 13.6 \\
E S=-0.21 \\
\text { Mean Pre/Post difference } \\
1.5 \pm 7.1 E S=0.21 \\
\text { Mean Pre/Post difference } \\
0.9 \pm 1.5 \\
E S=0.62\end{array}$ \\
\hline $\begin{array}{l}\text { Marcellis et al., } 2015 \\
\text { The Netherlands }\end{array}$ & Pre-Post study & $\begin{array}{l}18 \text { patients with Sarcoidosis } \\
\left(14 \sigma^{7} ;\right. \\
50.3 \pm 10.4 \text { years; } \mathrm{FEV}_{1} 93.6 \pm \\
17.0 \% \text { pred; FVC } 102.2 \pm \\
18.1 \% \text { pred; DLCO } 91.2 \pm \\
18.4 \% \text { pred })\end{array}$ & $\begin{array}{l}\text { Aerobic and strength } \\
\text { training } \\
40 \% \text { of } 1 \mathrm{RM} \\
60 \% \text { of maximal } \\
\text { walking speed in the } \\
6 \mathrm{MWT} \\
50 \% \text { of WRmax }\end{array}$ & $\begin{array}{l}13 \text { weeks } \\
3 \text { sessions/week } \\
1 \mathrm{~h} / \text { session }\end{array}$ & HR at rest (bpm) & $\begin{array}{l}\text { Pre } 82.7 \pm 13.1 \text { vs. Post } 77.1 \\
\pm 12.8, \mathrm{p}=0.11 \\
E S=-0.43\end{array}$ \\
\hline $\begin{array}{l}\text { Vainshelboim at al., } \\
2015 \\
\text { Israel }\end{array}$ & $\begin{array}{l}\text { Randomized controlled } \\
\text { trial }\end{array}$ & $\begin{array}{l}15 \text { patients with IPF }\left(10 \sigma^{7} ;\right. \\
68.8 \pm 6 \text { years; FVC } 66.1 \pm \\
14.8 \% \text { pred; DLCO } 48.6 \pm \\
17.2 \% \text { pred) } \\
\text { Arterial hypertension: } n=12 \\
\text { Coronary heart disease: } n=7 \\
\text { Pulmonary hypertension: } n=5\end{array}$ & $\begin{array}{l}\text { Aerobic and strength } \\
\text { training } \\
50 \%-70 \% \text { of WRpeak } \\
70 \%-90 \% \text { of average } \\
\text { walking speed in the } \\
6 \mathrm{MWT} \\
\text { Perceived exertion } \\
3-6 \text { in the mBorg }\end{array}$ & $\begin{array}{l}12 \text { weeks } \\
2 \text { sessions/week } \\
60 \mathrm{~min} / \text { session }\end{array}$ & & \\
\hline $\begin{array}{l}\text { Boström et al., } 2016 \\
\text { Sweden }\end{array}$ & $\begin{array}{l}\text { Randomized controlled } \\
\text { trial }\end{array}$ & $\begin{array}{l}18 \text { patients with Systemic } \\
\text { lupus erythematosus }\left(0 \sigma^{7} ; 52.0\right. \\
\pm 10.0 \text { years) } \\
\text { Arterial hypertension: } n=6\end{array}$ & $\begin{array}{l}\text { Pulmonary } \\
\text { rehabilitation } \\
65 \%-80 \% \text { of HRmax } \\
\text { Perceived exertion } \\
\text { 13-16 in the Borg }\end{array}$ & $\begin{array}{l}12 \text { weeks } \\
2 \text { sessions/week } \\
60 \mathrm{~min} / \text { session }\end{array}$ & $\begin{array}{l}\text { HR at rest } \\
\text { Blood pressure at rest }\end{array}$ & $\begin{array}{l}\text { Pre vs. Post, } \mathrm{p}=0.04 \\
\text { Pre vs. Post, } p>0.05\end{array}$ \\
\hline
\end{tabular}


Table 4. Cont.

\begin{tabular}{|c|c|c|c|c|c|c|}
\hline Study and Country & Study Design & Population & Intervention & $\begin{array}{l}\text { Duration and } \\
\text { Frequency }\end{array}$ & $\begin{array}{l}\text { Cardiovascular Outcomes } \\
\text { and Outcome Measures }\end{array}$ & $\begin{array}{c}\text { Results on Cardiovascular } \\
\text { Outcomes }\end{array}$ \\
\hline $\begin{array}{l}\text { Vainshelboim et al., } \\
2017 \\
\text { Israel }\end{array}$ & $\begin{array}{l}\text { Randomized controlled } \\
\text { trial }\end{array}$ & $\begin{array}{l}15 \text { patients with IPF }\left(100^{\top} ; 68.8\right. \\
\pm 6.0 \text { years; FVC } 66.1 \pm \\
14.8 \% \text { pred; DLCO } 48.6 \pm \\
17.2 \% \text { pred }) \\
\text { Arterial hypertension: } n=12 \\
\text { Coronary heart disease: } n=7 \\
\text { Pulmonary hypertension: } n=5\end{array}$ & $\begin{array}{l}\text { Aerobic and strength } \\
\text { training } \\
50 \%-70 \% \text { of WRpeak } \\
70 \%-90 \% \text { of average } \\
\text { walking speed in the } \\
6 \mathrm{MWT}\end{array}$ & $\begin{array}{l}12 \text { weeks } \\
2 \text { sessions/week } \\
60 \mathrm{~min} / \text { session }\end{array}$ & $\begin{array}{l}\text { HR (bpm) } \\
\text { HR reserve (bpm) } \\
\text { Systolic blood pressure } \\
\text { (mmHg) } \\
\text { Diastolic blood pressure } \\
\text { (mmHg) } \\
\text { Rate pressure product } \\
\text { (bpm/mmHg) } \\
\text { Left atrium diameter (cm) } \\
\text { Left atrium area (cm2) } \\
\text { Left ventricle posterior wall } \\
\text { thickness (cm) } \\
\text { Intra-ventricular septum } \\
\text { thickness (cm) } \\
\text { Left ventricle end systolic } \\
\text { diameter index (cm/m2) } \\
\text { Left ventricle end diastolic } \\
\text { diameter index (cm/m2) } \\
\text { Stroke volume (mL/beat) } \\
\text { Cardiac output (L/min) } \\
\text { Cardiac index (L/min/m2) }\end{array}$ & $\begin{array}{l}\text { Mean Pre/Post difference } \\
-2.4 \pm 9.1 \\
E S=-0.26 \\
\text { Mean Pre/Post difference } \\
6.7 \pm 11.0 \\
E S=0.61 \\
\text { Mean Pre/Post difference } \\
-2.9 \pm 13.6 \\
E S=-0.21 \\
\text { Mean Pre/Post difference } \\
1.5 \pm 7.1 \\
E S=0.21 \\
\text { Mean Pre/Post difference } \\
1685.0 \pm 3338.0 \\
E S=0.50 \\
\text { Mean Pre/Post difference } \\
0.0 \pm 0.5 \\
E S=0.04 \\
\text { Mean Pre/Post difference } \\
0.2 \pm 2.7 \\
E S=0.07 \\
\text { Mean Pre/Post difference } \\
0.0 \pm 0.1 \\
E S=0.30 \\
\text { Mean Pre/Post difference } \\
0.1 \pm 0.1 \\
E S=0.60 \\
\text { Mean Pre/Post difference } \\
-0.1 \pm 0.3 \\
E S=-0.40 \\
\text { Mean Pre/Post difference } \\
-0.1 \pm 0.3 \\
E S=-0.47 \\
\text { Mean Pre/Post difference } \\
-4.5 \pm 13.4 \\
E S=-0.34 \\
\text { Mean Pre/Post difference } \\
-0.4 \pm 0.8 \\
E S=-0.50 \\
\text { Mean Pre/Post difference } \\
-0.2 \pm 0.4 \\
T S \\
\end{array}$ \\
\hline
\end{tabular}


Table 4. Cont

\begin{tabular}{|c|c|c|c|c|c|c|}
\hline Study and Country & Study Design & Population & Intervention & $\begin{array}{l}\text { Duration and } \\
\text { Frequency }\end{array}$ & $\begin{array}{l}\text { Cardiovascular Outcomes } \\
\text { and Outcome Measures }\end{array}$ & $\begin{array}{c}\text { Results on Cardiovascular } \\
\text { Outcomes }\end{array}$ \\
\hline & & & & \multirow{11}{*}{1} & Ejection fraction (\%) & $\begin{array}{l}\text { Mean Pre/Post difference } \\
0.8 \pm 3.0 \\
E S=0.27\end{array}$ \\
\hline & & & & & Fractioning shortening (\%) & $\begin{array}{l}\text { Mean Pre/Post difference } \\
0.9 \pm 6.2 \\
E S=0.15\end{array}$ \\
\hline & & & & & $\begin{array}{l}\text { Earlier transmitral velocity } \\
\text { (E) }(\mathrm{ms})\end{array}$ & $\begin{array}{l}\text { Mean Pre/Post difference } \\
0.8 \pm 16.9 \\
E S=0.05\end{array}$ \\
\hline & & & & & $\begin{array}{l}\text { Late trasmitral velocity (A) } \\
(\mathrm{ms})\end{array}$ & $\begin{array}{l}\text { Mean Pre/Post difference } \\
5.1 \pm 20.7 \\
E S=0.25\end{array}$ \\
\hline & & & & & E/A ratio & $\begin{array}{l}\text { Mean Pre/Post difference } \\
0.0 \pm 0.4 \\
E S=0.00\end{array}$ \\
\hline & & & & & $\begin{array}{l}\text { Isovolumic relaxation time } \\
\text { (ms) }\end{array}$ & $\begin{array}{l}\text { Mean Pre/Post difference } \\
9.1 \pm 32.1 \\
E S=0.28 \\
\text { Mean Pre/Post difference }\end{array}$ \\
\hline & & & & & Deceleration time (ms) & $\begin{array}{l}11.0 \pm 52.7 \\
E S=0.21\end{array}$ \\
\hline & & & & & $\begin{array}{l}\text { Systolic pulmonary arterial } \\
\text { pressure }(\mathrm{mmHg})\end{array}$ & $\begin{array}{l}\text { Mean Pre/Post difference } \\
-0.5 \pm 6.8 \\
E S=-0.07\end{array}$ \\
\hline & & & & & $\begin{array}{l}\text { Peak circulatory power } \\
(\mathrm{mLO} / \mathrm{kg} / \mathrm{min} / \mathrm{mmHg})\end{array}$ & $\begin{array}{l}\text { Mean Pre/Post difference } \\
490.0 \pm 637.0 \\
E S=0.77\end{array}$ \\
\hline & & & & & $\begin{array}{l}\text { Peak cardiac power output } \\
\text { (W) }\end{array}$ & $\begin{array}{l}\text { Mean Pre/Post difference } \\
0.3 \pm 0.3 \\
E S=0.94\end{array}$ \\
\hline & & & & & $\begin{array}{l}\text { Peak stroke work } \\
\text { (mLO2/beat/mmHg) }\end{array}$ & $\begin{array}{l}\text { Mean Pre/Post difference } \\
221.0 \pm 343.0 \\
E S=0.64\end{array}$ \\
\hline
\end{tabular}


Table 4. Cont.

\begin{tabular}{|c|c|c|c|c|c|c|}
\hline Study and Country & Study Design & Population & Intervention & $\begin{array}{l}\text { Duration and } \\
\text { Frequency }\end{array}$ & $\begin{array}{l}\text { Cardiovascular Outcomes } \\
\text { and Outcome Measures }\end{array}$ & $\begin{array}{c}\text { Results on Cardiovascular } \\
\text { Outcomes }\end{array}$ \\
\hline $\begin{array}{l}\text { Naz et al., 2018a } \\
\text { Turkey }\end{array}$ & Pre-Post study & $\begin{array}{l}14 \text { patients with ILD }\left(50^{7} ; 63.0\right. \\
(53.0 ; 70.0) \text { years; FEV } 178.0 \\
(69.0 ; 83.0) \% \text { pred; FVC } 74.0 \\
(67.0 ; 78.0) \% \text { pred; DLCO } 40.0 \\
(19.0 ; 45.0) \% \text { pred) } \\
\text { Arterial hypertension: } n=7 \\
\text { Congestive heart failure: } n=2\end{array}$ & $\begin{array}{l}\text { Aerobic and strength } \\
\text { training } \\
80 \% \text { of peak walking } \\
\text { speed in the } 6 \mathrm{MWT} \\
70 \% \text { of WRmax } \\
\text { Dyspnoea and } \\
\text { perceived exertion } \\
4-6 \text { in the mBorg }\end{array}$ & $\begin{array}{l}12 \text { weeks } \\
2 \text { sessions/week } \\
60-90 \mathrm{~min} / \mathrm{session}\end{array}$ & & \\
\hline $\begin{array}{l}\text { Naz et al., 2018b } \\
\text { Turkey }\end{array}$ & $\begin{array}{l}\text { Randomized controlled } \\
\text { trial }\end{array}$ & $\begin{array}{l}9 \text { patients with Sarcoidosis } \\
\left(33.3 \% \sigma^{7} ;\right. \\
59.0(52.0 ; 64.0) \text { years; } \mathrm{FEV}_{1} \\
73.0(65.0 ; 85.0) \% \text { pred; FVC } \\
76.0(66.0 ; 90.0) \% \text { pred; DLCO } \\
45.0(36.0 ; 54.0) \% \text { pred })\end{array}$ & $\begin{array}{l}\text { Aerobic and strength } \\
\text { training } \\
80 \% \text { of the peak speed } \\
\text { in the } 6 \mathrm{MWT} \\
\text { Fatigue } 4-6 \text { in the } \\
\text { mBorg }\end{array}$ & $\begin{array}{l}12 \text { weeks } \\
2 \text { sessions/week }\end{array}$ & HR (bpm) & $\begin{array}{l}\text { Median Pre/Post difference } \\
0.0[-6.0 ; 5.0], p>0.05\end{array}$ \\
\hline
\end{tabular}

Data are presented as mean \pm standard deviation or median (interquartile range), unless otherwise stated. Legend: 6MWT, 6-min walk test; 1RM, one repetition maximum; \%pred,

percentage predicted; DLCO, diffusing capacity for carbon monoxide; ES, effect size; $\mathrm{FEV}_{1}$, forced expiratory volume in $1 \mathrm{~s}$; FVC, forced vital capacity; $\mathrm{HR}$, heart rate; HR $\mathrm{Hax}_{\text {, }}$ maximum

heart rate; ILD, interstitial lung disease; IPF, idiopathic pulmonary fibrosis; mBorg, modified Borg scale; WR, work rate; $W_{\text {max }}$, maximal work rate; WR peak, peak work rate. 
Most studies $(n=20 ; 71.4 \%)$ presented only small to moderate effects in the cardiovascular outcome measures reported. Standard deviation of RR intervals $[49,108](n=2 ; E S=[0.67 ; 2.64])$ and root mean square of successive RR interval differences $[49,57,108](n=3 ; E S=[0.69 ; 2.64])$ were the outcome measures presenting the larger effects. In patients with COPD, the effects of exercise training programmes on resting heart rate resulted in an overall pooled $E S$ of -0.23 ( $95 \%$ confidence interval -0.33 to -0.13 ) (Figure 5).

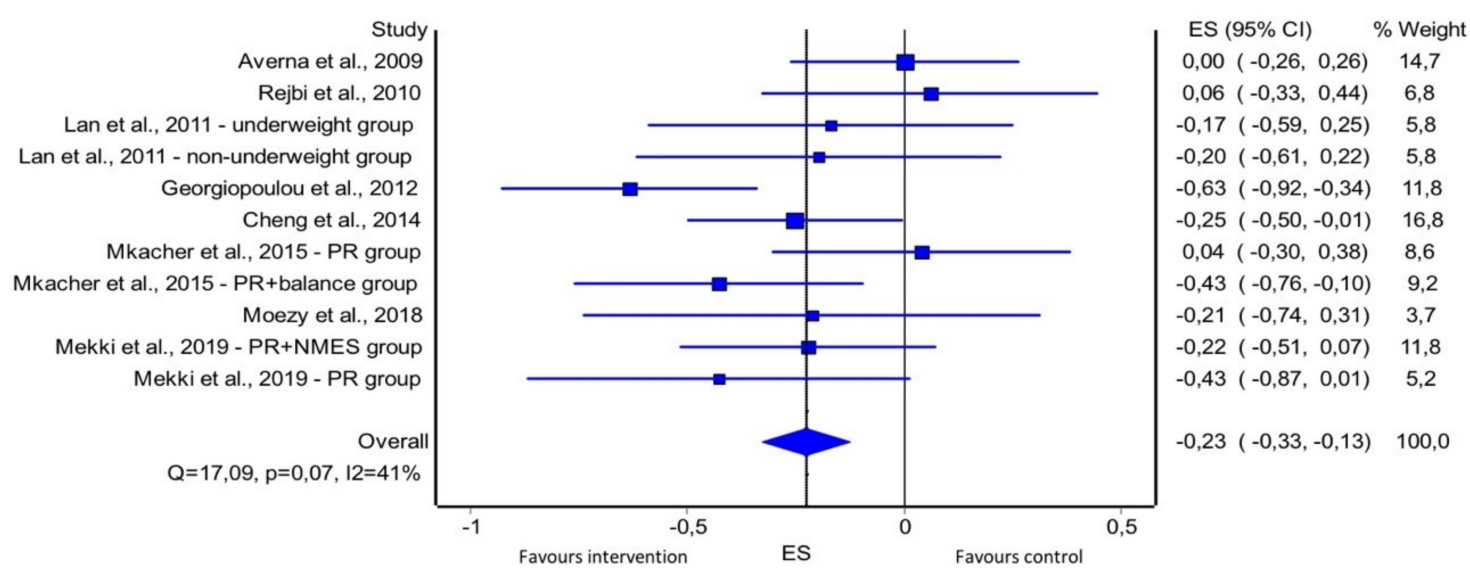

Figure 5. Forest plot of comparison control versus intervention in patients with COPD; outcome: resting heart rate. ES, effect size; NMES, neuromuscular electrical stimulation; PR, pulmonary rehabilitation.

Regarding the exercise programmes, most studies conducted in patients with COPD performed a pulmonary rehabilitation programme $[33,54,55,57,59,68,80,90,101,104,106,107,121,123,136,167](n=16)$ or an exercise programme combining aerobic and strength training $[38,43-45,54,86,129,130](n=8)$. Sessions were conducted 2-6 times per week and each session lasted from $15 \mathrm{~min}$ to $2 \mathrm{~h}$. Programme duration varied between 12 weeks and 18 months. A wide range of intensities was used to prescribe the exercise: $60 \%-80 \%$ of the maximum heart rate, $50 \%-100 \%$ of the peak or maximum oxygen uptake, $50 \%-125 \%$ of the peak or maximum workload, $35 \%-75 \%$ of one-repetition maximum, dyspnoea and perceived exertion levels between 3-6 on the modified Borg scale and 12-16 on the Borg scale. None of the studies specified any adjustments to tailor the exercise programmes to patients' cardiovascular comorbidities. Only one study [108] described adjusting the training programme in different mesocycles in order to improve specific cardiovascular outcomes.

Studies conducted in patients with asthma performed either an exercise programme combining aerobic and strength training for 3 months [189] or aerobic training for 6 months [186]. Sessions occurred 3 times/week, for 30 min each, at an intensity of $60 \%-80 \%$ of the maximum heart rate. No specific adjustments to improve specific cardiovascular outcomes were reported.

In patients with ILD, most studies conducted exercise programmes combining aerobic and strength training [201-203,207-209] $(n=6)$. The majority of programmes lasted for 12 weeks with 2 sessions/week [196,198,202,203,207-209] ( $n=7)$. Sessions had a duration of 60-90 min and exercise was prescribed at an intensity of $65 \%-85 \%$ of maximum heart rate, $50 \%-90 \%$ of peak workload, dyspnoea and perceived exertion levels between 3 and 6 on the modified Borg scale. None of the studies specified any adjustments to tailor the exercise programmes to patients' cardiovascular comorbidities.

\section{Discussion}

To the best of the authors' knowledge, this is the first comprehensive overview of the scientific literature summarizing (i) the eligibility criteria in terms of cardiovascular disease used to select patients with chronic respiratory disease to exercise training studies, (ii) the impact of at least 3 months of exercise training on cardiovascular outcomes, and (iii) adjustments made to tailor exercise training prescription to patients with cardiovascular comorbidities. It was found that (i) in the majority of the 
studies (58.9\%) patients with cardiovascular comorbidities were excluded a priori, (ii) there is limited evidence about the impact of exercise training on cardiovascular outcomes in patients with chronic respiratory diseases, and (iii) none of the studies explicitly mentioned how to tailor exercise training modalities in light of cardiovascular comorbidities.

A large diversity was found regarding the cardiovascular conditions that are used as exclusion criteria in exercise-related research. Interestingly, the majority of the exclusion criteria reported (34/45) are not considered contraindications to exercise training. Indeed, just a minority of the studies $(18.3 \%)$ excluded only patients with acute/unstable cardiovascular disease that contraindicated participation in exercise training. Most studies excluded patients with both stable and unstable cardiovascular comorbidities, although at least $20 \%-50 \%$ of the patients with COPD, asthma or ILD present cardiovascular comorbidities $[5,7,18,20,210,211]$. Thus, by excluding patients with cardiovascular comorbidities or any other comorbidity that does not present any contraindication to perform exercise training, translation of knowledge to clinical practice can only be done for a subset, or sometimes even a minority, of patients. This finding might have a far-reaching consequence, namely that current knowledge (including clinical guidelines) is disease-centred and, thus, inadequate to sufficiently support/guide clinicians on how to prescribe exercise for patients with chronic respiratory diseases and multiple chronic conditions [13,212]. Furthermore, some of the criteria reported (e.g., cardiovascular disease) were too vague to allow understanding of which conditions were really excluded and over $20 \%$ of the included studies did not report any information concerning to eligibility criteria, even though this is key information to ensure clarity and transparency of the research [213].

Exercise training programmes in patients with cardiovascular comorbidities resulted in significant improvements in general reported outcomes, namely symptoms, functionality, exercise capacity, muscle strength and health-related quality of life, comparable to the ones usually found in respiratory patients [22]. However, regarding cardiovascular outcomes, in the majority of the studies (71.4\%) only small to moderate effects were found, with the larger effects being reported for heart rate variability measurements $(E S=[-0.78 ; 2.64])$ and blood lipid profile $(E S=[-2.31 ; 0.62])$. Additionally, a small but significant overall effect of exercise training programmes on resting heart rate of patients with COPD was found. These results are yet not inferior to the ones previously reported for patients with cardiovascular diseases, in whom beneficial effects of exercise training have been found for heart rate variability and heart rate recovery [214], and inconsistent but significant and modest effects have been reported for arterial blood pressure and blood lipid profile [214-217]. We hypothesized that several reasons might be contributing to the limited effects found. First, most studies have not reported any specific adjustments in the exercise prescription to tailor the programme to patients' cardiovascular comorbidities, although it is plausible that they have made some adjustments without specific reporting in the published paper. It is known that cardiovascular conditions require specific considerations when formulating the exercise plan [22], and different recommendations exist based on the prevalent cardiovascular disease (e.g., coronary artery disease, congestive heart failure, peripheral arterial disease, pulmonary arterial hypertension) and its severity [218]. Indeed, it is mandatory to tailor exercise duration, frequency, mode, intensity and monitoring to patients' specificities and needs, clinical conditions, cardiovascular phenotype (risk factors and diseases), fitness level, medication intake (beta blockers, statins, glinides, sulfonylurea), abnormal responses to exercise (myocardial ischemia, atrial fibrillation, ventricular tachycardia) and rehabilitation goals [1,218-221]. Moreover, the impact of exercise training relies on this proper tailoring of the exercise programme, since it has been shown that different exercise prescriptions result in significant differences in clinical outcomes [221]. Future studies should therefore assess the impact of exercise programmes specifically tailored to patients with co-occurring respiratory disease and cardiovascular comorbidities [7] and report the intervention in detail. Second, guidelines for cardiac rehabilitation from the leading scientific societies recommend that exercise should progress from moderate to vigorous intensity, three times per week [21]. Nevertheless, in some of the included studies, patients exercised at lower intensities and/or fewer times per week, which might have also contributed to the relative lack of effects since the minimum 
dose of exercise for cardiovascular benefits ( $>150 \mathrm{~min} /$ week of endurance training, energy expenditure $1000-2000 \mathrm{kcal} /$ week) might have not been reached [218,222]. Third, some of the included studies only used strength training in their exercise programmes. Indeed, strength training has been recommended in patients with cardiovascular diseases, but as an adjunct to aerobic training, the last being a core component in these patients' rehabilitation [21]. From these observations, it became clear that current exercise prescription to patients with COPD, asthma or ILD with cardiovascular comorbidities is far from optimal and deserves significant reconsideration. Nonetheless, digital support on how to prescribe exercise in these patients in accordance to all the different clinical guidelines for different cardiovascular diseases is available, and thus could be used to support health professionals [218]. Lastly, most studies including patients with cardiovascular comorbidities only focused on the assessment of resting heart rate. Although this is a relevant outcome measure and results from meta-analysis in patients with COPD favour intervention, recommendations for patients with cardiovascular diseases advocate a more comprehensive assessment, including outcomes such as arterial blood pressure, blood lipid profile or echocardiography, that are also more in line with the aims of rehabilitation in these patients $[223,224]$. Therefore, outcomes should be better targeted to patients' cardiovascular comorbidities [19].

Besides the known prevalence and increased risk of morbidity and mortality that cardiovascular comorbidities impose on patients with chronic respiratory diseases $[7,211]$, only three studies $[196,207,209]$ (all conducted in the last 5 years) included patients with cardiovascular comorbidities and assessed cardiovascular outcome measures. This denotes the current gap in the literature regarding exercise programmes and emphasises the need for specific studies focusing on cardiovascular outcomes in these patients.

This systematic review has a number of limitations that need to be acknowledged. First, as it was anticipated that a large number of studies would be found, only exercise programmes lasting at least 12 weeks were included, which might have led to the loss of other relevant studies. Nevertheless, 12 weeks has been recommended as the minimum exercise duration required to reach benefits in patients with cardiovascular disease [25]. Second, as only few studies including patients with ILD were found, all types of ILD were grouped, although different types of ILD present different characteristics and possibly different cardiovascular comorbidities and responses to exercise training programmes. Third, most of the included studies were of weak quality. Nonetheless, since in exercise interventions blinding of participants is impossible and patients are usually referred by physicians to ensure their safety, it was virtually impossible to ensure strong quality in the quality assessment tool used.

\section{Conclusions}

Although a large number of studies explored the effects of at least 3 months of exercise training in patients with chronic respiratory diseases, only few included patients with cardiovascular comorbidities. Limited effects of the exercise programmes were found on cardiovascular outcome measures, possibly due to the lack of tailoring of the exercise training prescription and comprehensiveness of the cardiovascular outcome measures. Future studies focusing on patients with combined respiratory and cardiovascular diseases and exploring the effects of exercise programmes specifically tailored to these patients are needed to bridge the gap in the literature.

Author Contributions: All authors made substantial contributions to the concept and design of the work and approved the submitted version (conceptualization, D.H. and C.B.; methodology, A.M, K.Q. and C.B.; formal analysis, A.M.; investigation, A.M., K.Q. and A.O.; data curation, A.M., K.Q. and A.O.; writing-original draft preparation, A.M.; writing-review and editing, K.Q., A.O., C.K., A.M., D.H., C.B.; supervision, C.B.; project administration, C.B.).

Funding: This research received no external funding.

Acknowledgments: We acknowledge the support of the European Respiratory Society - ERS Short-Term Research Fellowship October 2018 (STRTF201810-00469).

Conflicts of Interest: The authors declare no conflict of interest. 


\section{Appendix A Search Strategy}

\section{A1. PubMed}

\#1 Search ("chronic obstructive pulmonary disease" OR "copd" OR "asthma" OR "interstitial lung diseas*" OR "ILD" OR "parenchymal lung disease" OR "parenchymal lung disorder" OR "pulmonary fibrosis" OR "sarcoidosis" OR "interstitial pneumonia" OR "connective tissue disease" OR "collagen vascular disease" OR "occupational lung disease" OR "hypersensitivity pneumonitis" OR "asbestosis" OR "silicosis" OR "beryliosis" OR "respiratory bronchiolitis" OR "desquamative interstitial pneumonia" OR "cryptogenic organising pneumonia" OR "lymphoid interstitial pneumonia" OR "pleuroparenchymal fibroelastosis" OR "pneumoconiosis" OR "extrinsic allergic alveolitis" OR "Iatrogenic interstitial lung disease" OR "post-infectious interstitial lung disease" OR "granulomatose" OR "systemic sclerosis" OR "polymyositis" OR "dermatomyositis" OR "systemic lupus erythematosus" OR "Hamman-Rich syndrome" OR "bagassosis" OR "histiocytosis" OR "fibrotic interstitial lung disease" OR "fibrotic lung disease")

\#2 Search ("exercise" OR "walking" OR "aerobic training" OR “endurance training" OR "interval training" OR "high-intensity training" OR "resistance training" OR "strength training")

\#3 Search ("Pulmonary Disease, Chronic Obstructive" OR "Asthma" OR "Lung Diseases, Interstitial" OR "Pulmonary Fibrosis" OR "Sarcoidosis" OR "Connective Tissue Diseases" OR "Alveolitis, Extrinsic Allergic" OR "Asbestosis" OR "Silicosis" OR "Pneumoconiosis" OR "Scleroderma, Systemic" OR "Polymyositis" OR “Dermatomyositis" OR “Lupus Erythematosus, Systemic" OR "Histiocytosis"))

\#4 Search ("Exercise" OR "Walking" OR "Endurance Training" OR "Resistance Training")

\#5 Search (\#1 AND \#2 [Title/Abstract]) OR (\#3 AND \#4 [MeSH Terms])

\section{A2. Cochrane, Scopus and Web of Science}

\#1 Search ("chronic obstructive pulmonary disease" OR copd OR asthma OR "interstitial lung diseas" OR ILD OR "parenchymal lung disease" OR "parenchymal lung disorder" OR "pulmonary fibrosis" OR sarcoidosis OR "interstitial pneumonia" OR "connective tissue disease" OR "collagen vascular disease" OR "occupational lung disease" OR "hypersensitivity pneumonitis" OR asbestosis OR silicosis OR beryliosis OR "respiratory bronchiolitis" OR "desquamative interstitial pneumonia" OR "cryptogenic organising pneumonia" OR "lymphoid interstitial pneumonia" OR "pleuroparenchymal fibroelastosis" OR pneumoconiosis OR "extrinsic allergic alveolitis" OR "Iatrogenic interstitial lung disease" OR "post-infectious interstitial lung disease" OR granulomatose OR "systemic sclerosis" OR polymyositis OR dermatomyositis OR "systemic lupus erythematosus" OR "Hamman-Rich syndrome" OR bagassosis OR histiocytosis OR "fibrotic interstitial lung disease" OR "fibrotic lung disease")

\#2 Search ("exercise" OR "walking" OR "aerobic training" OR "endurance training" OR "interval training" OR "high-intensity training" OR "resistance training" OR "strength training")

\#3 Search (\#1 AND \#2)

\section{References}

1. Armstrong, M.; Vogiatzis, I. Personalized exercise training in chronic lung diseases. Respirology 2019, 24, 854-862. [CrossRef] [PubMed]

2. Bousquet, J.; Kiley, J.; Bateman, E.; Viegi, G.; Cruz, A.; Khaltaev, N.; Khaled, N.A.; Baena-Cagnani, C.; Barreto, M.; Billo, N. Prioritised research agenda for prevention and control of chronic respiratory diseases. Eur. Respir. J. 2010, 36, 995-1001. [CrossRef] [PubMed]

3. Prince, M.J.; Wu, F.; Guo, Y.; Robledo, L.M.G.; O'Donnell, M.; Sullivan, R.; Yusuf, S. The burden of disease in older people and implications for health policy and practice. Lancet 2015, 385, 549-562. [CrossRef]

4. World Health Organization. Global Health Estimates 2016: Deaths by Cause, Age, Sex, by Country and by Region, 2000-2016; WHO: Geneva, Switzerland, 2018. 
5. Mannino, D.M.; Thorn, D.; Swensen, A.; Holguin, F. Prevalence and outcomes of diabetes, hypertension and cardiovascular disease in COPD. Eur. Respir. J. 2008, 32, 962-969. [CrossRef] [PubMed]

6. Boulet, L.-P.; Boulay, M.-È. Asthma-related comorbidities. Expert Rev. Respir. Med. 2011, 5, $377-393$. [CrossRef] [PubMed]

7. Franssen, F.M.; Rochester, C.L. Comorbidities in patients with COPD and pulmonary rehabilitation: Do they matter? Eur Respir. Soc 2014, 23, 131-141. [CrossRef] [PubMed]

8. Houben-Wilke, S.; Spruit, M.A.; Uszko-Lencer, N.H.; Otkinska, G.; Vanfleteren, L.E.; Jones, P.W.; Wouters, E.F.; Franssen, F.M. Echocardiographic abnormalities and their impact on health status in patients with COPD referred for pulmonary rehabilitation. Respirology 2017, 22, 928-934. [CrossRef]

9. Hyldgaard, C.; Hilberg, O.; Bendstrup, E. How does comorbidity influence survival in idiopathic pulmonary fibrosis? Respir. Med. 2014, 108, 647-653. [CrossRef]

10. King, C.; Nathan, S.D. Identification and treatment of comorbidities in idiopathic pulmonary fibrosis and other fibrotic lung diseases. Curr. Opin. Pulm. Med. 2013, 19, 466-473. [CrossRef]

11. Soriano, J.B.; Visick, G.T.; Muellerova, H.; Payvandi, N.; Hansell, A.L. Patterns of comorbidities in newly diagnosed COPD and asthma in primary care. Chest 2005, 128, 2099-2107. [CrossRef]

12. Triest, F.J.; Singh, S.J.; Vanfleteren, L.E. Cardiovascular risk, chronic obstructive pulmonary disease and pulmonary rehabilitation: Can we learn from cardiac rehabilitation? Chronic Respir. Dis. 2016, 13, $286-294$. [CrossRef] [PubMed]

13. Houben-Wilke, S.; Triest, F.J.; Franssen, F.M.; Janssen, D.J.; Wouters, E.F.; Vanfleteren, L.E. Revealing methodological challenges in chronic obstructive pulmonary disease studies assessing comorbidities: A narrative review. Chronic Obstr. Pulm. Dis. J. Copd. Found. 2019, 6, 166. [CrossRef] [PubMed]

14. Divo, M.; Cote, C.; de Torres, J.P.; Casanova, C.; Marin, J.M.; Pinto-Plata, V.; Zulueta, J.; Cabrera, C.; Zagaceta, J.; Hunninghake, G. Comorbidities and risk of mortality in patients with chronic obstructive pulmonary disease. Am. J. Respir. Crit. Care Med. 2012, 186, 155-161. [CrossRef] [PubMed]

15. Kreuter, M.; Ehlers-Tenenbaum, S.; Palmowski, K.; Bruhwyler, J.; Oltmanns, U.; Muley, T.; Heussel, C.P.; Warth, A.; Kolb, M.; Herth, F.J. Impact of comorbidities on mortality in patients with idiopathic pulmonary fibrosis. PLoS ONE 2016, 11, e0151425. [CrossRef] [PubMed]

16. Miller, J.; Edwards, L.D.; Agustí, A.; Bakke, P.; Calverley, P.M.; Celli, B.; Coxson, H.O.; Crim, C.; Lomas, D.A.; Miller, B.E. Comorbidity, systemic inflammation and outcomes in the ECLIPSE cohort. Respir. Med. 2013, 107, 1376-1384. [CrossRef] [PubMed]

17. Terzano, C.; Conti, V.; Di Stefano, F.; Petroianni, A.; Ceccarelli, D.; Graziani, E.; Mariotta, S.; Ricci, A.; Vitarelli, A.; Puglisi, G. Comorbidity, hospitalization, and mortality in COPD: Results from a longitudinal study. Lung 2010, 188, 321-329. [CrossRef] [PubMed]

18. Cazzola, M.; Calzetta, L.; Bettoncelli, G.; Cricelli, C.; Romeo, F.; Matera, M.G.; Rogliani, P. Cardiovascular disease in asthma and COPD: A population-based retrospective cross-sectional study. Respir. Med. 2012, 106, 249-256. [CrossRef]

19. Crisafulli, E.; Costi, S.; Luppi, F.; Cirelli, G.; Cilione, C.; Coletti, O.; Fabbri, L.M.; Clini, E.M. Role of comorbidities in a cohort of patients with COPD undergoing pulmonary rehabilitation. Thorax 2008, 63, 487-492. [CrossRef]

20. Margaritopoulos, G.A.; Antoniou, K.M.; Wells, A.U. Comorbidities in interstitial lung diseases. Eur. Respir. Rev. 2017, 26, 160027. [CrossRef]

21. Price, K.J.; Gordon, B.A.; Bird, S.R.; Benson, A.C. A review of guidelines for cardiac rehabilitation exercise programmes: Is there an international consensus? Eur. J. Prev. Cardiol. 2016, 23, 1715-1733. [CrossRef]

22. Spruit, M.A.; Singh, S.J.; Garvey, C.; ZuWallack, R.; Nici, L.; Rochester, C.; Hill, K.; Holland, A.E.; Lareau, S.C.; Man, W.D.-C. An official American Thoracic Society/European Respiratory Society statement: Key concepts and advances in pulmonary rehabilitation. Am. J. Respir. Crit. Care Med. 2013, 188, e13-e64. [CrossRef] [PubMed]

23. Hornikx, M.; Van Remoortel, H.; Lehouck, A.; Mathieu, C.; Maes, K.; Gayan-Ramirez, G.; Decramer, M.; Troosters, T.; Janssens, W. Vitamin D supplementation during rehabilitation in COPD: A secondary analysis of a randomized trial. Respir. Res. 2012, 13, 84. [CrossRef] [PubMed]

24. Moher, D.; Liberati, A.; Tetzlaff, J.; Altman, D.G.; Group, P. Preferred reporting items for systematic reviews and meta-analyses: The PRISMA statement. PLoS Med. 2009, 6, e1000097. [CrossRef] [PubMed] 
25. Man, W.D.; Chowdhury, F.; Taylor, R.S.; Evans, R.A.; Doherty, P.; Singh, S.J.; Booth, S.; Thomason, D.; Andrews, D.; Lee, C. Building consensus for provision of breathlessness rehabilitation for patients with chronic obstructive pulmonary disease and chronic heart failure. Chronic Respir. Dis. 2016, 13, $229-239$. [CrossRef] [PubMed]

26. Rochester, C.L.; Vogiatzis, I.; Holland, A.E.; Lareau, S.C.; Marciniuk, D.D.; Puhan, M.A.; Spruit, M.A.; Masefield, S.; Casaburi, R.; Clini, E.M. An official American Thoracic Society/European Respiratory Society policy statement: Enhancing implementation, use, and delivery of pulmonary rehabilitation. Am. J. Respir. Crit. Care Med. 2015, 192, 1373-1386. [CrossRef]

27. Thomas, B.; Ciliska, D.; Dobbins, M.; Micucci, S. A process for systematically reviewing the literature: Providing the research evidence for public health nursing interventions. World Views Evid. Based Nurs. 2004, 1, 176-184. [CrossRef]

28. Landis, J.R.; Koch, G.G. The measurement of observer agreement for categorical data. Biometrics 1977, 33, 159-174. [CrossRef]

29. Morris, S.B.; DeShon, R.P. Combining effect size estimates in meta-analysis with repeated measures and independent-groups designs. Psychol. Methods 2002, 7, 105. [CrossRef]

30. Cohen, J. Statistical Power Analysis for the Behavioral Sciences, 2nd ed.; Erlbaum Associates: Hillsdale, MI, USA, 1988.

31. Abd El-Kader, S.M.; Al-Jiffri, O.H. Exercise alleviates depression related systemic inflammation in chronic obstructive pulmonary disease patients. Afr. Health Sci. 2016, 16, 1078-1088. [CrossRef]

32. Abd El-Kader, S.M.; Al-Jiffri, O.H.; Al-Jiffri, H.O. Aerobic exercise training modulates bone mineral status in patients with chronic obstructive pulmonary disease. Eur. J. Gen. Med. 2016, 13, 51-54. [CrossRef]

33. Almadana Pacheco, V.; Pavon Masa, M.; Gomez-Bastero Fernandez, A.P.; Muniz Rodriguez, A.M.; Tallon Moreno, R.; Montemayor Rubio, T. Patient Profile of Drop-Outs From a Pulmonary Rehabilitation Program. Arch. De Bronconeumol. 2017, 53, 257-262. [CrossRef] [PubMed]

34. Altenburg, W.A.; Duiverman, M.L.; Ten Hacken, N.H.; Kerstjens, H.A.; de Greef, M.H.; Wijkstra, P.J.; Wempe, J.B. Changes in the endurance shuttle walk test in COPD patients with chronic respiratory failure after pulmonary rehabilitation: The minimal important difference obtained with anchor- and distribution-based method. Respir. Res. 2015, 16, 27. [CrossRef] [PubMed]

35. Amin, S.; Abrazado, M.; Quinn, M.; Storer, T.W.; Tseng, C.H.; Cooper, C.B. A controlled study of community-based exercise training in patients with moderate COPD. BMC Pulm. Med. 2014, 14, 125. [CrossRef] [PubMed]

36. Arnardóttir, R.H.; Boman, G.; Larsson, K.; Hedenström, H.; Emtner, M. Interval training compared with continuous training in patients with COPD. Respir. Med. 2007, 101, 1196-1204. [CrossRef] [PubMed]

37. Averna, T.; Brunelli, S.; Delussu, A.S.; Porcacchia, P.; Lucarelli, E.; Polidori, L.; Traballesi, M. Effects of a moderately intensive, 12-week training program on participants over 60 years of age with chronic obstructive pulmonary disease. Med. Dello Sport 2009, 62, 299-313.

38. Barakat, S.; Michele, G.; George, P.; Nicole, V.; Guy, A. Outpatient pulmonary rehabilitation in patients with chronic obstructive pulmonary disease. Int. J. Chronic Obstr. Pulm. Dis. 2008, 3, 155-162.

39. Bendstrup, K.E.; Jensen, J.I.; Holm, S.; Bengtsson, B. Out-patient rehabilitation improves activities of daily living, quality of life and exercise tolerance in chronic obstructive pulmonary disease. Eur. Respir. J. 1997, 10, 2801-2806. [CrossRef] [PubMed]

40. Bernard, S.; Whittom, F.; Leblanc, P.; Jobin, J.; Belleau, R.; Berube, C.; Carrier, G.; Maltais, F. Aerobic and strength training in patients with chronic obstructive pulmonary disease. Am. J. Respir. Crit. Care Med. 1999, 159, 896-901. [CrossRef]

41. Berry, M.J.; Adair, N.E.; Sevensky, K.S.; Quinby, A.; Lever, H.M. Inspiratory muscle training and whole-body reconditioning in chronic obstructive pulmonary disease. Am. J. Respir. Crit. Care Med. 1996, 153, 1812-1816. [CrossRef]

42. Berry, M.J.; Jack Rejeski, W.; Adair, N.E.; Zaccaro, D. Exercise rehabilitation and chronic obstructive pulmonary disease stage. Am. J. Respir. Crit. Care Med. 1999, 160, 1248-1253. [CrossRef]

43. Berry, M.J.; Rejeski, W.J.; Adair, N.E.; Ettinger Jr, W.H.; Zaccaro, D.J.; Sevick, M.A. A randomized, controlled trial comparing long-term and short-term exercise in patients with chronic obstructive pulmonary disease. J. Cardiopulm. Rehabil. 2003, 23, 60-68. [CrossRef] [PubMed] 
44. Berry, M.J.; Rejeski, W.J.; Miller, M.E.; Adair, N.E.; Lang, W.; Foy, C.G.; Katula, J.A. A lifestyle activity intervention in patients with chronic obstructive pulmonary disease. Respir. Med. 2010, 104, 829-839. [CrossRef] [PubMed]

45. Berry, M.J.; Sheilds, K.L.; Adair, N.E. Comparison of Effects of Endurance and Strength Training Programs in Patients with COPD. COPD 2018, 15, 192-199. [CrossRef] [PubMed]

46. Bingisser, R.M.; Joos, L.; Frühauf, B.; Caravatti, M.; Knoblauch, A.; Villiger, P.M. Pulmonary rehabilitation in outpatients with asthma or chronic obstructive lung disease. Swiss Med Wkly. 2001, 131, 407-411. [PubMed]

47. Bisca, G.W.; Proenca, M.; Salomao, A.; Hernandes, N.A.; Pitta, F. Minimal detectable change of the London chest activity of daily living scale in patients with COPD. J. Cardiopulm. Rehabil. Prev. 2014, 34, $213-216$. [CrossRef]

48. Boeselt, T.; Nell, C.; Lutteken, L.; Kehr, K.; Koepke, J.; Apelt, S.; Veith, M.; Beutel, B.; Spielmanns, M.; Greulich, T.; et al. Benefits of High-Intensity Exercise Training to Patients with Chronic Obstructive Pulmonary Disease: A Controlled Study. Respir. Int. Rev. Thorac. Dis. 2017, 93, 301-310. [CrossRef] [PubMed]

49. Borghi-Silva, A.; Mendes, R.G.; Trimer, R.; Oliveira, C.R.; Fregonezi, G.A.; Resqueti, V.R.; Arena, R.; Sampaio-Jorge, L.M.; Costa, D. Potential effect of 6 versus 12-weeks of physical training on cardiac autonomic function and exercise capacity in chronic obstructive pulmonary disease. Eur. J. Phys. Rehabil. Med. 2015, 51, 211-221.

50. Burtin, C.; Langer, D.; van Remoortel, H.; Demeyer, H.; Gosselink, R.; Decramer, M.; Dobbels, F.; Janssens, W.; Troosters, T. Physical Activity Counselling during Pulmonary Rehabilitation in Patients with COPD: A Randomised Controlled Trial. PLoS ONE 2015, 10, e0144989. [CrossRef]

51. Burtin, C.; Saey, D.; Saglam, M.; Langer, D.; Gosselink, R.; Janssens, W.; Decramer, M.; Maltais, F.; Troosters, T. Effectiveness of exercise training in patients with COPD: The role of muscle fatigue. Eur. Respir. J. 2012, 40, 338-344. [CrossRef]

52. Cambach, W.; Chadwick-Straver, R.V.; Wagenaar, R.C.; van Keimpema, A.R.; Kemper, H.C. The effects of a community-based pulmonary rehabilitation programme on exercise tolerance and quality of life: A randomized controlled trial. Eur. Respir. J. 1997, 10, 104-113. [CrossRef]

53. Camillo, C.A.; Laburu, V.D.M.; Gonalves, N.S.; Cavalheri, V.; Tomasi, F.P.; Hernandes, N.A.; Ramos, D.; Marquez Vanderlei, L.C.; Cipulo Ramos, E.M.; Probst, V.S.; et al. Improvement of heart rate variability after exercise training and its predictors in COPD. Respir. Med. 2011, 105, 1054-1062. [CrossRef] [PubMed]

54. Campos, A.A.; Cabrera, R.O.; Arancibia, H.F. Respiratory rehabilitation in COPD patients: Experience in a rural primary health care center. Rev. Chil. De Enferm. Respir. 2015, 31, 77-85.

55. Charikiopoulou, M.; Nikolaidis, P.T.; Knechtle, B.; Rosemann, T.; Rapti, A.; Trakada, G. Subjective and Objective Outcomes in Patients With COPD After Pulmonary Rehabilitation-The Impact of Comorbidities. Front. Physiol. 2019, 10, 286. [CrossRef] [PubMed]

56. Chen, R.; Chen, R.; Chen, X.; Chen, L. Effect of endurance training on expiratory flow limitation and dynamic hyperinflation in patients with stable chronic obstructive pulmonary disease. Intern. Med. J. 2014, 44, 791-800. [CrossRef] [PubMed]

57. Cheng, S.T.; Wu, Y.K.; Yang, M.C.; Huang, C.Y.; Huang, H.C.; Chu, W.H.; Lan, C.C. Pulmonary rehabilitation improves heart rate variability at peak exercise, exercise capacity and health-related quality of life in chronic obstructive pulmonary disease. Heart Lung J. Crit. Care 2014, 43, 249-255. [CrossRef] [PubMed]

58. Clark, C.J.; Cochrane, L.M.; Mackay, E.; Paton, B. Skeletal muscle strength and endurance in patients with mild COPD and the effects of weight training. Eur. Respir. J. 2000, 15, 92-97. [CrossRef] [PubMed]

59. Corhay, J.L.; Nguyen, D.; Duysinx, B.; Graas, C.; Pirnay, F.; Bury, T.; Louis, R. Should we exclude elderly patients with chronic obstructive pulmonary disease from a long-time ambulatory pulmonary rehabilitation programme? J. Rehabil. Med. 2012, 44, 466-472. [CrossRef] [PubMed]

60. Cortopassi, F.; Castro, A.A.; Porto, E.F.; Colucci, M.; Fonseca, G.; Torre-Bouscoulet, L.; Iamonti, V.; Jardim, J.R. Comprehensive exercise training improves ventilatory muscle function and reduces dyspnea perception in patients with COPD. Monaldi Arch. Chest Dis. Arch. Monaldi Per Le Mal. Del Torace 2009, 71, 106-112. [CrossRef]

61. Covey, M.K.; Collins, E.G.; Reynertson, S.I.; Dilling, D.F. Resistance training as a preconditioning strategy for enhancing aerobic exercise training outcomes in COPD. Respir. Med. 2014, 108, 1141-1152. [CrossRef] 
62. Covey, M.K.; McAuley, E.; Kapella, M.C.; Collins, E.G.; Alex, C.G.; Berbaum, M.L.; Larson, J.L. Upper-Body Resistance Training and Self-Efficacy Enhancement in COPD. J. Pulm. Respir. Med. 2012, 1 (Suppl. 9.). [CrossRef]

63. Cox, N.J.M.; Hendricks, J.C.; Binkhorst, R.A.; van Herwaarden, C.L.A. A pulmonary rehabilitation program for patients with asthma and mild chronic obstructive pulmonary diseases (COPD). Lung Int. J. Lungs Airw. Breath 1993, 171, 235-244. [CrossRef]

64. Crimi, C.; Heffler, E.; Augelletti, T.; Campisi, R.; Noto, A.; Vancheri, C.; Crimi, N. Utility of ultrasound assessment of diaphragmatic function before and after pulmonary rehabilitation in COPD patients. Int. J. Chronic Obstr. Pulm. Dis. 2018, 13, 3131-3139. [CrossRef] [PubMed]

65. Cruz, J.; Brooks, D.; Marques, A. Impact of feedback on physical activity levels of individuals with chronic obstructive pulmonary disease during pulmonary rehabilitation: A feasibility study. Chron. Respir. Dis. 2014, 11, 191-198. [CrossRef] [PubMed]

66. Cruz, J.; Brooks, D.; Marques, A. Walk2Bactive: A randomised controlled trial of a physical activity-focused behavioural intervention beyond pulmonary rehabilitation in chronic obstructive pulmonary disease. Chron. Respir. Dis. 2016, 13, 57-66. [CrossRef] [PubMed]

67. Da Costa, C.C.; de Azeredo Lermen, C.; Colombo, C.; Canterle, D.B.; Machado, M.L.L.; Kessler, A.; Teixeira, P.J.Z. Effect of a Pulmonary Rehabilitation Program on the levels of anxiety and depression and on the quality of life of patients with chronic obstructive pulmonary disease. Rev. Port. Pneumol. 2014, 20, 299-304. [CrossRef] [PubMed]

68. Da Silva Cardoso, M.C.; Sayão, L.B.; Souza, R.M.P.; De Melo Marinho, P.E. Pulmonary rehabilitation and whole-body vibration in chronic obstructive pulmonary disease. Mot. Rev. De Educ. Fis. 2016, 22, 44-50. [CrossRef]

69. da Silva, G.P.; Nascimento, F.A.; Macedo, T.P.; Morano, M.T.; Mesquita, R.; Pereira, E.D. Religious coping and religiosity in patients with COPD following pulmonary rehabilitation. Int. J. Chronic Obstr. Pulm. Dis. 2018, 13, 175-181. [CrossRef] [PubMed]

70. De Alencar Silva, B.S.; Gobbo, L.A.; Freire, A.P.C.F.; Trevisan, I.B.; Silva, I.G.; Ramos, E.M.C. Effects of a resistance training with elastic tubing in strength, quality of life and dypsnea in patients with chronic obstructive pulmonary disease. J. Phys. Educ. 2016, 27. [CrossRef]

71. De Oliveira, J.C.M.; Leitao, F.S.S.; Sampaio, L.M.M.; de Oliveira, A.C.N.; Hirata, R.P.; Costa, D.; Donner, C.F.; de Oliveira, L.V.F. Outpatient vs. home-based pulmonary rehabilitation in COPD: A randomized controlled trial. Multidiscip. Respir. Med. 2010, 5, 401-408. [CrossRef]

72. De Souza, Y.; da Silva, K.M.; Condesso, D.; Figueira, B.; Filho, A.J.N.; Rufino, R.; Gosselink, R.; da Costa, C.H. Use of a home-based manual as part of a pulmonary rehabilitation program. Respir. Care 2018, 63, 1485-1491. [CrossRef]

73. Demeyer, H.; Burtin, C.; Van Remoortel, H.; Hornikx, M.; Langer, D.; Decramer, M.; Gosselink, R.; Janssens, W.; Troosters, T. Standardizing the Analysis of Physical Activity in Patients With COPD Following a Pulmonary Rehabilitation Program. Chest 2014, 146, 318-327. [CrossRef] [PubMed]

74. Dourado, V.S.; Tanni, S.E.; Antunes, L.C.O.; Paiva, S.A.R.; Campana, A.O.; Renno, A.C.M.; Godoy, I. Effect of three exercise programs on patients with chronic obstructive pulmonary disease. Braz. J. Med Biol. Res. 2009, 42, 263-271. [CrossRef] [PubMed]

75. Dourado, V.Z.; Antunes, L.C.O.; Tanni, S.E.; Godoy, I. Factors associated with the minimal clinically important difference for health-related quality of life after physical conditioning in patients with COPD. J. Bras. De Pneumol. 2009, 35, 846-853. [CrossRef] [PubMed]

76. Effing, T.; Zielhuis, G.; Kerstjens, H.; van der Valk, P.; van der Palen, J. Community based physiotherapeutic exercise in COPD self-management: A randomised controlled trial. Respir. Med. 2011, 105, 418-426. [CrossRef] [PubMed]

77. Emtner, M.; Hallin, R.; Arnardottir, R.H.; Janson, C. Effect of physical training on fat-free mass in patients with chronic obstructive pulmonary disease (COPD). Upsala J. Med. Sci. 2015, 120, 52-58. [CrossRef] [PubMed]

78. Endo, Y.; Dobashi, K.; Uga, D.; Kato, D.; Nakazawa, R.; Sakamoto, M.; Fueki, M.; Makino, S. Effect of 12-month rehabilitation with low loading program on chronic respiratory disease. J. Phys. Ther. Sci. 2016, 28, 1032-1035. [CrossRef] [PubMed]

79. Enea, C.; Schmitt, N.; Dugú, B.; Boisseau, N.; Le Creff, C.; Denjean, A. Oxidative stress in patients with chronic obstructive pulmonary disease after aerobic exercise training. Sci. Sports 2005, 20, 48-50. [CrossRef] 
80. Engel, R.M.; Gonski, P.; Beath, K.; Vemulpad, S. Medium term effects of including manual therapy in a pulmonary rehabilitation program for chronic obstructive pulmonary disease (COPD): A randomized controlled pilot trial. J. Man. Manip. Ther. 2016, 24, 80-89. [CrossRef]

81. Etnier, J.L.; Berry, M. Fluid intelligence in an older COPD sample after short- or long-term exercise. Med. Sci. Sports Exerc. 2001, 33, 1620-1628. [CrossRef]

82. Felcar, J.M.; Probst, V.S.; de Carvalho, D.R.; Merli, M.F.; Mesquita, R.; Vidotto, L.S.; Ribeiro, L.R.G.; Pitta, F. Effects of exercise training in water and on land in patients with COPD: A randomised clinical trial. Physiotherapy 2018, 104, 408-416. [CrossRef]

83. Fernandes, J.R.; da Silva, C.; da Silva, A.G.; Pinto, R.M.D.; Duarte, A.J.D.; Carvalho, C.R.; Benard, G. Effect of an Exercise Program on Lymphocyte Proliferative Responses of COPD Patients. Lung 2018, 196, 271-276. [CrossRef] [PubMed]

84. Fischer, M.J.; Scharloo, M.; Abbink, J.; van 't Hul, A.; van Ranst, D.; Rudolphus, A.; Weinman, J.; Rabe, K.F.; Kaptein, A.A. Concerns about exercise are related to walk test results in pulmonary rehabilitation for patients with COPD. Int. J. Behav. Med. 2012, 19, 39-47. [CrossRef] [PubMed]

85. Foy, C.G.; Rejeski, W.J.; Berry, M.J.; Zaccaro, D.; Woodard, C.M. Gender moderates the effects of exercise therapy on health-related quality of life among COPD patients. Chest 2001, 119, 70-76. [CrossRef]

86. Fuld, J.P.; Kilduff, L.P.; Neder, J.A.; Pitsiladis, Y.; Lean, M.E.J.; Ward, S.A.; Cotton, M.M. Creatine supplementation during pulmonary rehabilitation in chronic obstructive pulmonary disease. Thorax 2005, 60, 531-537. [CrossRef] [PubMed]

87. Fuller, L.M.; Button, B.; Tarrant, B.; Steward, R.; Bennett, L.; Snell, G.; Holland, A.E. Longer Versus Shorter Duration of Supervised Rehabilitation After Lung Transplantation: A Randomized Trial. Arch. Phys. Med. Rehabil. 2017, 98, 220-226. [CrossRef] [PubMed]

88. Gayle, R.C.; Spitler, D.L.; Karper, W.B.; Jaeger, R.M.; Rice, S.N. Psychological changes in exercising COPD patients. Int. J. Rehabil. Res. Int. Z. Fur Rehabil. Rev. Int. De Rech. De Readapt. 1988, 11, 335-342. [CrossRef]

89. Georgiadou, O.; Vogiatzis, I.; Stratakos, G.; Koutsoukou, A.; Golemati, S.; Aliverti, A.; Roussos, C.; Zakynthinos, S. Effects of rehabilitation on chest wall volume regulation during exercise in COPD patients. Eur. Respir. J. 2007, 29, 284-291. [CrossRef] [PubMed]

90. Georgiopoulou, V.V.; Dimopoulos, S.; Sakellariou, D.; Papazachou, O.; Gerovasili, V.; Tasoulis, A.; Agapitou, V.; Vogiatzis, I.; Roussos, C.; Nanas, S. Cardiopulmonary rehabilitation enhances heart rate recovery in patients with COPD. Respir. Care 2012, 57, 2095-2103. [CrossRef]

91. Godoy, R.F.; Teixeira, P.J.; Becker Junior, B.; Michelli, M.; Godoy, D.V. Long-term repercussions of a pulmonary rehabilitation program on the indices of anxiety, depression, quality of life and physical performance in patients with COPD. J. Bras. De Pneumol. Publ. Da Soc. Bras. De Pneumol. E Tisilogia 2009, 35, 129-136.

92. Goldstein, R.S.; Gort, E.H.; Avendano, M.A.; Stubbing, D.; Guyatt, G.H. Randomised controlled trial of respiratory rehabilitation. Lancet 1994, 344, 1394-1397. [CrossRef]

93. Grosbois, J.M.; Lamblin, C.; Lemaire, B.; Chekroud, H.; Dernis, J.M.; Douay, B.; Fortin, F. Long-term benefits of exercise maintenance after outpatient rehabilitation program in patients with chronic obstructive pulmonary disease. J. Cardiopulm. Rehabil. 1999, 19, 216-225. [CrossRef] [PubMed]

94. Guell, R.; Casan, P.; Belda, J.; Sangenis, M.; Morante, F.; Guyatt, G.H.; Sanchis, J. Long-term effects of outpatient rehabilitation of COPD—A randomized trial. Chest 2000, 117, 976-983. [CrossRef] [PubMed]

95. Harrison, S.L.; Beauchamp, M.K.; Sibley, K.; Araujo, T.; Romano, J.; Goldstein, R.S.; Brooks, D. Minimizing the evidence-practice gap-A prospective cohort study incorporating balance training into pulmonary rehabilitation for individuals with chronic obstructive pulmonary disease. BMC Pulm. Med. 2015, 15, 73. [CrossRef] [PubMed]

96. Hoogendoorn, M.; van Wetering, C.R.; Schols, A.M.; Rutten-van Molken, M.P. Is INTERdisciplinary COMmunity-based COPD management (INTERCOM) cost-effective? Eur. Respir. J. 2010, 35, $79-87$. [CrossRef] [PubMed]

97. Houben-Wilke, S.; Janssen, D.J.A.; Franssen, F.M.E.; Vanfleteren, L.; Wouters, E.F.M.; Spruit, M.A. Contribution of individual COPD assessment test (CAT) items to CAT total score and effects of pulmonary rehabilitation on CAT scores. Health Qual. Life Outcomes 2018, 16, 205. [CrossRef] [PubMed]

98. Jacome, C.; Marques, A. Short- and Long-term Effects of Pulmonary Rehabilitation in Patients With Mild COPD: A COMPARISON WITH PATIENTS WITH MODERATE TO SEVERE COPD. J. Cardiopulm. Rehabil. Prev. 2016, 36, 445-453. [CrossRef] 
99. Jácome, C.; Marques, A. Impact of pulmonary rehabilitation in subjects with mild COPD. Respir. Care 2014, 59, 1577-1582. [CrossRef]

100. Kamal, M.A.W.; Nambi, S.G.; Mahmoud, M.Z. Impact of Resisted Exercise on Chronic Obstructive Pulmonary Disease (COPD) in Elderly Patients in Alkharj, Saudi Arabia. Int. J. Med Res. Health Sci. 2016, 5, 187-195.

101. Kanao, K.; Shiraishi, M.; Higashimoto, Y.; Maeda, K.; Sugiya, R.; Okajima, S.; Chiba, Y.; Yamagata, T.; Terada, K.; Fukuda, K.; et al. Factors associated with the effect of pulmonary rehabilitation on physical activity in patients with chronic obstructive pulmonary disease. Geriatr. Gerontol. Int. 2017, 17, 17-23. [CrossRef]

102. Kavoura, P.; Kostikas, K.; Tselebis, A.; Bratis, D.; Kosmas, E.; Alchanatis, M.; Koulouris, N.G.; Bakakos, P.; Loukides, S. Changes in BODE Quartiles After Pulmonary Rehabilitation Do Not Predict 2-Year Survival in Patients With COPD. J. Cardiopulm. Rehabil. Prev. 2016, 36, 62-67. [CrossRef]

103. Kongsgaard, M.; Backer, V.; Jorgensen, K.; Kjaer, M.; Beyer, N. Heavy resistance training increases muscle size, strength and physical function in elderly male COPD-patients-A pilot study. Respir. Med. 2004, 98, 1000-1007. [CrossRef] [PubMed]

104. Lan, C.C.; Chu, W.H.; Yang, M.C.; Lee, C.H.; Wu, Y.K.; Wu, C.P. Benefits of pulmonary rehabilitation in patients with COPD and normal exercise capacity. Respir. Care 2013, 58, 1482-1488. [CrossRef] [PubMed]

105. Lan, C.C.; Huang, H.C.; Yang, M.C.; Lee, C.H.; Huang, C.Y.; Wu, Y.K. Pulmonary rehabilitation improves subjective sleep quality in COPD. Respir. Care 2014, 59, 1569-1576. [CrossRef] [PubMed]

106. Lan, C.C.; Yang, M.C.; Huang, H.C.; Wu, C.W.; Su, W.L.; Tzeng, I.S.; Wu, Y.K. Serial changes in exercise capacity, quality of life and cardiopulmonary responses after pulmonary rehabilitation in patients with chronic obstructive pulmonary disease. Heart Lung J. Crit. Care 2018, 47, 477-484. [CrossRef] [PubMed]

107. Lan, C.C.; Yang, M.C.; Lee, C.H.; Huang, Y.C.; Huang, C.Y.; Huang, K.L.; Wu, Y.K. Pulmonary rehabilitation improves exercise capacity and quality of life in underweight patients with chronic obstructive pulmonary disease. Respirology 2011, 16, 276-283. [CrossRef] [PubMed]

108. Leite, M.R.; Ramos, E.M.; Kalva-Filho, C.A.; Freire, A.P.; de Alencar Silva, B.S.; Nicolino, J.; de Toledo-Arruda, A.C.; Papoti, M.; Vanderlei, L.C.; Ramos, D. Effects of 12 weeks of aerobic training on autonomic modulation, mucociliary clearance, and aerobic parameters in patients with COPD. Int. J. Chronic Obstr. Pulm. Dis. 2015, 10, 2549-2557. [CrossRef] [PubMed]

109. López Varela, M.V.; Anido, T.; Larrosa, M. Functional status and survival in patients with chronic obstructive pulmonary disease following pulmonary rehabilitation. Arch. De Bronconeumol. 2006, 42, 434-439. [CrossRef]

110. Lox, C.L.; Freehill, A.J. Impact of pulmonary rehabilitation on self-efficacy, quality of life, and exercise tolerance. Rehabil. Psychol. 1999, 44, 208-221. [CrossRef]

111. Maa, S.H.; Gauthier, D.; Turner, M. Acupressure as an adjunct to a pulmonary rehabilitation program. J. Cardiopulm. Rehabil. 1997, 17, 268-276. [CrossRef]

112. Magadle, R.; McConnell, A.K.; Beckerman, M.; Weiner, P. Inspiratory muscle training in pulmonary rehabilitation program in COPD patients. Respir. Med. 2007, 101, 1500-1505. [CrossRef]

113. Maltais, F.; LeBlanc, P.; Jobin, J.; Berube, C.; Bruneau, J.; Carrier, L.; Breton, M.J.; Falardeau, G.; Belleau, R. Intensity of training and physiologic adaptation in patients with chronic obstructive pulmonary disease. Am. J. Respir. Crit. Care Med. 1997, 155, 555-561. [CrossRef] [PubMed]

114. Maltais, F.; LeBlanc, P.; Simard, C.; Jobin, J.; Berube, C.; Bruneau, J.; Carrier, L.; Belleau, R. Skeletal muscle adaptation to endurance training in patients with chronic obstructive pulmonary disease. Am. J. Respir. Crit. Care Med. 1996, 154, 442-447. [CrossRef] [PubMed]

115. Mandigout, S.; Antonini, M.T.; Laforge, Q.; Lemaire, F.; Dalmay, E.; Bouteille, B. Effects of training rehabilitation on the physical capacity of patients suffering from chronic obstructive pulmonary disease. Sci. Sports 2007, 22, 300-301. [CrossRef]

116. Marques, A.; Gabriel, R.; Jácome, C.; Cruz, J.; Brooks, D.; Figueiredo, D. Development of a family-based pulmonary rehabilitation programme: An exploratory study. Disabil. Rehabil. 2015, 37, 1340-1346. [CrossRef] [PubMed]

117. Marques, A.; Jacome, C.; Cruz, J.; Gabriel, R.; Brooks, D.; Figueiredo, D. Family-based psychosocial support and education as part of pulmonary rehabilitation in COPD: A randomized controlled trial. Chest 2015, 147, 662-672. [CrossRef] [PubMed] 
118. Marques, A.; Jacome, C.; Cruz, J.; Gabriel, R.; Figueiredo, D. Effects of a pulmonary rehabilitation program with balance training on patients with COPD. J. Cardiopulm. Rehabil. Prev. 2015, 35, 154-158. [CrossRef] [PubMed]

119. Márquez-Martín, E.; Ruiz, F.O.; Ramos, P.C.; López-Campos, J.L.; Azcona, B.V.; Cortés, E.B. Randomized trial of non-invasive ventilation combined with exercise training in patients with chronic hypercapnic failure due to chronic obstructive pulmonary disease. Respir. Med. 2014, 108, 1741-1751. [CrossRef]

120. Marquis, K.; Maltais, F.; Lacasse, Y.; Lacourciere, Y.; Fortin, C.; Poirier, P. Effects of aerobic exercise training and irbesartan on blood pressure and heart rate variability in patients with chronic obstructive pulmonary disease. Can. Respir. J. 2008, 15, 355-360. [CrossRef]

121. Mekki, M.; Paillard, T.; Sahli, S.; Tabka, Z.; Trabelsi, Y. Effect of adding neuromuscular electrical stimulation training to pulmonary rehabilitation in patients with chronic obstructive pulmonary disease: Randomized clinical trial. Clin. Rehabil. 2019, 33, 195-206. [CrossRef]

122. Milani, R.V.; Lavie, C.J. Disparate effects of out-patient cardiac and pulmonary rehabilitation programs on work efficiency and peak aerobic capacity in patients with coronary disease or severe obstructive pulmonary disease. J. Cardiopulm. Rehabil. 1998, 18, 17-22. [CrossRef]

123. Mkacher, W.; Mekki, M.; Tabka, Z.; Trabelsi, Y. Effect of 6 Months of Balance Training During Pulmonary Rehabilitation in Patients With COPD. J. Cardiopulm. Rehabil. Prev. 2015, 35, 207-213. [CrossRef]

124. Moezy, A.; Erfani, A.; Mazaherinezhad, A.; Mousavi, S.A.J. Downhill walking influence on physical condition and quality of life in patients with COPD: A randomized controlled trial. Med. J. Islam. Repub. Iran 2018, 32, 49. [CrossRef]

125. Neunhauserer, D.; Steidle-Kloc, E.; Weiss, G.; Kaiser, B.; Niederseer, D.; Hartl, S.; Tschentscher, M.; Egger, A.; Schonfelder, M.; Lamprecht, B.; et al. Supplemental Oxygen During High-Intensity Exercise Training in Nonhypoxemic Chronic Obstructive Pulmonary Disease. Am. J. Med. 2016, 129, 1185-1193. [CrossRef]

126. Ortega, F.; Toral, J.; Cejudo, P.; Villagomez, R.; Sanchez, H.; Castillo, J.; Montemayor, T. Comparison of effects of strength and endurance training in patients with chronic obstructive pulmonary disease. Am. J. Respir. Crit. Care Med. 2002, 166, 669-674. [CrossRef]

127. Osadnik, C.R.; Loeckx, M.; Louvaris, Z.; Demeyer, H.; Langer, D.; Rodrigues, F.M.; Janssens, W.; Vogiatzis, I.; Troosters, T. The likelihood of improving physical activity after pulmonary rehabilitation is increased in patients with COPD who have better exercise tolerance. Int. J. Chronic Obstr. Pulm. Dis. 2018, 13, 3515-3527. [CrossRef]

128. Panton, L.B.; Golden, J.; Broeder, C.E.; Browder, K.D.; Cestaro-Seifer, D.J.; Seifer, F.D. The effects of resistance training on functional outcomes in patients with chronic obstructive pulmonary disease. Eur. J. Appl. Physiol. 2004, 91, 443-449. [CrossRef]

129. Papp, M.E.; Wändell, P.E.; Lindfors, P.; Nygren-Bonnier, M. Effects of yogic exercises on functional capacity, lung function and quality of life in participants with obstructive pulmonary disease: A randomized controlled study. Eur. J. Phys. Rehabil. Med. 2017, 53, 447-461.

130. Pereira, E.D.B.; Viana, C.S.; Taunay, T.C.E.; Sales, P.U.; Lima, J.W.O.; Holanda, M.A. Improvement of Cognitive Function After a Three-Month Pulmonary Rehabilitation Program for COPD Patients. Lung 2011, 189, 279-285. [CrossRef]

131. Pitta, F.; Troosters, T.; Probst, V.S.; Langer, D.; Decramer, M.; Gosselink, R. Are patients with COPD more active after pulmonary rehabilitation? Chest 2008, 134, 273-280. [CrossRef]

132. Polkey, M.I.; Qiu, Z.H.; Zhou, L.; Zhu, M.D.; Wu, Y.X.; Chen, Y.Y.; Ye, S.P.; He, Y.S.; Jiang, M.; He, B.T.; et al. Tai Chi and Pulmonary Rehabilitation Compared for Treatment-Naive Patients With COPD: A Randomized Controlled Trial. Chest 2018, 153, 1116-1124. [CrossRef]

133. Probst, V.S.; Kovelis, D.; Hernandes, N.A.; Camillo, C.A.; Cavalheri, V.; Pitta, F. Effects of 2 exercise training programs on physical activity in daily life in patients with COPD. Respir. Care 2011, 56, 1799-1807. [CrossRef]

134. Probst, V.S.; Troosters, T.; Pitta, F.; Decramer, M.; Gosselink, R. Cardiopulmonary stress during exercise training in patients with COPD. Eur. Respir. J. 2006, 27, 1110-1118. [CrossRef]

135. Radom-Aizik, S.; Kaminski, N.; Hayek, S.; Halkin, H.; Cooper, D.M.; Ben-Dov, I. Effects of exercise training on quadriceps muscle gene expression in chronic obstructive pulmonary disease. J. Appl. Physiol. 2007, 102, 1976-1984. [CrossRef] 
136. Rejbi, I.B.; Trabelsi, Y.; Chouchene, A.; Ben Turkia, W.; Ben Saad, H.; Zbidi, A.; Kerken, A.; Tabka, Z. Changes in six-minute walking distance during pulmonary rehabilitation in patients with COPD and in healthy subjects. Int. J. Chronic Obstr. Pulm. Dis. 2010, 5, 209-215. [CrossRef]

137. Rinaldo, N.; Bacchi, E.; Coratella, G.; Vitali, F.; Milanese, C.; Rossi, A.; Schena, F.; Lanza, M. Effects of Combined Aerobic-Strength Training vs Fitness Education Program in COPD Patients. Int. J. Sports Med. 2017, 38, 1001-1008. [CrossRef]

138. Rizk, A.K.; Wardini, R.; Chan-Thim, E.; Bacon, S.L.; Lavoie, K.L.; Pepin, V. Acute responses to exercise training and relationship with exercise adherence in moderate chronic obstructive pulmonary disease. Chron. Respir. Dis. 2015, 12, 329-339. [CrossRef]

139. Roman, M.; Larraz, C.; Gomez, A.; Ripoll, J.; Mir, I.; Miranda, E.Z.; Macho, A.; Thomas, V.; Esteva, M. Efficacy of pulmonary rehabilitation in patients with moderate chronic obstructive pulmonary disease: A randomized controlled trial. BMC Fam. Pract. 2013, 14, 21. [CrossRef]

140. Santana, V.T.S.; Squassoni, S.D.; Neder, J.A.; Fiss, E. Influence of current smoking on adherence and responses to pulmonary rehabilitation in patients with COPD. Braz. J. Phys. Ther. 2010, 14, 16-23. [CrossRef]

141. Scherer, Y.K.; Schmieder, L.E. The effect of a pulmonary rehabilitation program on self-efficacy, perception of dyspnea, and physical endurance. Heart Lung J. Crit. Care 1997, 26, 15-22. [CrossRef]

142. Scherer, Y.K.; Schmieder, L.E.; Shimmel, S. The effects of education alone and in combination with pulmonary rehabilitation on self-efficacy in patients with COPD. Rehabil. Nurs. Off. J. Assoc. Rehabil. Nurses 1998, 23, 71-77. [CrossRef]

143. Sciriha, A.; Lungaro-Mifsud, S.; Bonello, A.; Agius, T.; Scerri, J.; Ellul, B.; Fenech, A.; Camilleri, L.; Montefort, S. Systemic inflammation in COPD is not influenced by pulmonary rehabilitation. Eur. J. Physiother. 2017, 19, 194-200. [CrossRef]

144. Sciriha, A.; Lungaro-Mifsud, S.; Scerri, J.; Bilocca, D.; Fsadni, C.; Fsadni, P.; Gerada, E.; Gouder, C.; Camilleri, L.; Montefort, S. Pulmonary rehabilitation in chronic obstructive pulmonary disease: Outcomes in a 12 week programme. Eur. J. Physiother. 2015, 17, 215-223. [CrossRef]

145. Sciriha, A.; Lungaro-Mifsud, S.; Scerri, J.; Magro, R.; Camilleri, L.; Montefort, S. Health status of COPD patients undergoing pulmonary rehabilitation: A comparative responsiveness of the CAT and SGRQ. Chron. Respir. Dis. 2017, 14, 352-359. [CrossRef]

146. Silva, B.S.A.; Lira, F.S.; Rossi, F.E.; Ramos, D.; Uzeloto, J.S.; Freire, A.; de Lima, F.F.; Gobbo, L.A.; Ramos, E.M.C. Inflammatory and Metabolic Responses to Different Resistance Training on Chronic Obstructive Pulmonary Disease: A Randomized Control Trial. Front. Physiol. 2018, 9, 262. [CrossRef]

147. Silva, B.S.A.; Ramos, D.; Bertolini, G.N.; Freire, A.; Leite, M.R.; Camillo, C.A.; Gobbo, L.A.; Ramos, E.M.C. Resistance exercise training improves mucociliary clearance in subjects with COPD: A randomized clinical trial. Pulmonology 2019. [CrossRef]

148. Skumlien, S.; Aure Skogedal, E.; Skrede Ryg, M.; Bjortuft, O. Endurance or resistance training in primary care after in-patient rehabilitation for COPD? Respir. Med. 2008, 102, 422-429. [CrossRef]

149. Solanes, I.; Guell, R.; Casan, P.; Sotomayor, C.; Gonzalez, A.; Feixas, T.; Gonzalez, M.; Guyatt, G. Duration of pulmonary rehabilitation to achieve a plateau in quality of life and walk test in COPD. Respir. Med. 2009, 103, 722-728. [CrossRef]

150. Spencer, L.M.; Alison, J.A.; McKeough, Z.J. A Survey of Opinions and Attitudes Toward Exercise Following a 12-month Maintenance Exercise Program for People with COPD. Cardiopulm. Phys. Ther. J. 2013, 24, 30-35. [CrossRef]

151. Spielmanns, M.; Fuchs-Bergsma, C.; Winkler, A.; Fox, G.; Krüger, S.; Baum, K. Effects of oxygen supply during training on subjects with COPD who are normoxemic at rest and during exercise: A blinded randomized controlled trial. Respir. Care 2015, 60, 540-548. [CrossRef]

152. Spruit, M.A.; Gosselink, R.; Troosters, T.; De Paepe, K.; Decramer, M. Resistance versus endurance training in patients with COPD and peripheral muscle weakness. Eur. Respir. J. 2002, 19, 1072-1078. [CrossRef]

153. Spruit, M.A.; Gosselink, R.; Troosters, T.; Kasran, A.; Van Vliet, M.; Decramer, M. Low-grade systemic inflammation and the response to exercise training in patients with advanced COPD. Chest 2005, 128, 3183-3190. [CrossRef]

154. Stav, D.; Raz, M.; Shpirer, I. Three years of pulmonary rehabilitation: Inhibit the decline in airflow obstruction, improves exercise endurance time, and body-mass index, in chronic obstructive pulmonary disease. BMC Pulm. Med. 2009, 9, 26. [CrossRef] 
155. Steinsbekk, A.; Lomundal, B.K. Three-year follow-up after a two-year comprehensive pulmonary rehabilitation program. Chron. Respir. Dis. 2009, 6, 5-11. [CrossRef]

156. Strijbos, J.H.; Postma, D.S.; van Altena, R.; Gimeno, F.; Koeter, G.H. Feasibility and effects of a home-care rehabilitation program in patients with chronic obstructive pulmonary disease. J. Cardiopulm. Rehabil. 1996, 16, 386-393. [CrossRef]

157. Strijbos, J.H.; Postma, D.S.; vanAltena, R.; Gimeno, F.; Koeter, G.H. A comparison between an outpatient hospital-based pulmonary rehabilitation program and a home-care pulmonary rehabilitation program in patients with COPD - A follow-up of 18 months. Chest 1996, 109, 366-372. [CrossRef]

158. Theander, K.; Jakobsson, P.; Jorgensen, N.; Unosson, M. Effects of pulmonary rehabilitation on fatigue, functional status and health perceptions in patients with chronic obstructive pulmonary disease: A randomized controlled trial. Clin. Rehabil. 2009, 23, 125-136. [CrossRef]

159. Theodorakopoulou, E.P.; Gennimata, S.A.; Harikiopoulou, M.; Kaltsakas, G.; Palamidas, A.; Koutsoukou, A.; Roussos, C.; Kosmas, E.N.; Bakakos, P.; Koulouris, N.G. Effect of pulmonary rehabilitation on tidal expiratory flow limitation at rest and during exercise in COPD patients. Respir. Physiol. Neurobiol. 2017, 238, 47-54. [CrossRef]

160. Toledo, A.; Borghi-Silva, A.; Sampaio, L.M.; Ribeiro, K.P.; Baldissera, V.; Costa, D. The impact of noninvasive ventilation during the physical training in patients with moderate-to-severe chronic obstructive pulmonary disease (COPD). Clinics 2007, 62, 113-120. [CrossRef]

161. Trappenburg, J.C.; Troosters, T.; Spruit, M.A.; Vandebrouck, N.; Decramer, M.; Gosselink, R. Psychosocial conditions do not affect short-term outcome of multidisciplinary rehabilitation in chronic obstructive pulmonary disease. Arch. Phys. Med. Rehabil. 2005, 86, 1788-1792. [CrossRef]

162. Troosters, T.; Gosselink, R.; Decramer, M. Short- and long-term effects of outpatient rehabilitation in patients with chronic obstructive pulmonary disease: A randomized trial. Am. J. Med. 2000, 109, 207-212. [CrossRef]

163. Troosters, T.; Gosselink, R.; Decramer, M. Exercise training in COPD: How to distinguish responders from nonresponders. J. Cardiopulm. Rehabil. 2001, 21, 10-17. [CrossRef]

164. Tselebis, A.; Bratis, D.; Pachi, A.; Moussas, G.; Ilias, I.; Harikiopoulou, M.; Theodorakopoulou, E.; Dumitru, S.; Kosmas, E.; Vgontzas, A.; et al. A pulmonary rehabilitation program reduces levels of anxiety and depression in COPD patients. Multidiscip. Respir. Med. 2013, 8. [CrossRef]

165. Van de Bool, C.; Rutten, E.P.A.; van Helvoort, A.; Franssen, F.M.E.; Wouters, E.F.M.; Schols, A. A randomized clinical trial investigating the efficacy of targeted nutrition as adjunct to exercise training in COPD. J. Cachexia Sarcopenia Muscle 2017, 8, 748-758. [CrossRef]

166. Van Helvoort, H.A.; de Boer, R.C.; van de Broek, L.; Dekhuijzen, R.; Heijdra, Y.F. Exercises commonly used in rehabilitation of patients with chronic obstructive pulmonary disease: Cardiopulmonary responses and effect over time. Arch. Phys. Med. Rehabil. 2011, 92,111-117. [CrossRef]

167. Vasilopoulou, M.; Papaioannou, A.I.; Kaltsakas, G.; Louvaris, Z.; Chynkiamis, N.; Spetsioti, S.; Kortianou, E.; Genimata, S.A.; Palamidas, A.; Kostikas, K.; et al. Home-based maintenance tele-rehabilitation reduces the risk for acute exacerbations of COPD, hospitalisations and emergency department visits. Eur. Respir. J. 2017, 49. [CrossRef]

168. Voduc, N.; Tessier, C.; Sabri, E.; Fergusson, D.; Lavallee, L.; Aaron, S.D. Effects of oxygen on exercise duration in chronic obstructive pulmonary disease patients before and after pulmonary rehabilitation. Can. Respir. J. 2010, 17, e14-e19. [CrossRef]

169. Vogiatzis, I.; Nanas, S.; Roussos, C. Interval training as an alternative modality to continuous exercise in patients with COPD. Eur. Respir. J. 2002, 20, 12-19. [CrossRef]

170. Vogiatzis, I.; Williamson, A.F.; Miles, J.; Taylor, I.K. Physiological response to moderate exercise workloads in a pulmonary rehabilitation program in patients with varying degrees of airflow obstruction. Chest 1999, 116, 1200-1207. [CrossRef]

171. Vonbank, K.; Strasser, B.; Mondrzyk, J.; Marzluf, B.A.; Richter, B.; Losch, S.; Nell, H.; Petkov, V.; Haber, P. Strength training increases maximum working capacity in patients with COPD - Randomized clinical trial comparing three training modalities. Respir. Med. 2012, 106, 557-563. [CrossRef]

172. Wada, J.T.; Borges-Santos, E.; Porras, D.C.; Paisani, D.M.; Cukier, A.; Lunardi, A.C.; Carvalho, C.R. Effects of aerobic training combined with respiratory muscle stretching on the functional exercise capacity and thoracoabdominal kinematics in patients with COPD: A randomized and controlled trial. Int. J. Chronic Obstr. Pulm. Dis. 2016, 11, 2691-2700. [CrossRef] 
173. Wadell, K.; Henriksson-Larsen, K.; Lundgren, R.; Sundelin, G. Group training in patients with COPD—Long-term effects after decreased training frequency. Disabil. Rehabil. 2005, 27, 571-581. [CrossRef] [PubMed]

174. Wadell, K.; Sundelin, G.; Henriksson-Larsen, K.; Lundgren, R. High intensity physical group training in water-An effective training modality for patients with COPD. Respir. Med. 2004, 98, 428-438. [CrossRef] [PubMed]

175. Weiner, P.; Azgad, Y.; Ganam, R. Inspiratory muscle training combined with general exercise reconditioning in patients with COPD. Chest 1992, 102, 1351-1356. [CrossRef] [PubMed]

176. Wijkstra, P.J.; Van Altena, R.; Kraan, J.; Otten, V.; Postma, D.S.; Koeter, G.H. Quality of life in patients with chronic obstructive pulmonary disease improves after rehabilitation at home. Eur. Respir. J. 1994, 7, 269-273. [CrossRef] [PubMed]

177. Wijkstra, P.J.; van der Mark, T.W.; Kraan, J.; van Altena, R.; Koeter, G.H.; Postma, D.S. Effects of home rehabilitation on physical performance in patients with chronic obstructive pulmonary disease (COPD). Eur. Respir. J. 1996, 9, 104-110. [CrossRef] [PubMed]

178. Wright, P.R.; Heck, H.; Langenkamp, H. Effects of a resistance training on pulmonary function and performance measurements in patients with chronic obstructive pulmonary disease. Eur. J. Sport Sci. 2003, 3 , 1-10. [CrossRef]

179. Wu, W.; Liu, X.; Liu, J.; Li, P.; Wang, Z. Effectiveness of water-based Liuzijue exercise on respiratory muscle strength and peripheral skeletal muscle function in patients with COPD. Int. J. Chronic Obstr. Pulm. Dis. 2018, 13, 1713-1726. [CrossRef]

180. Xu, J.; He, S.; Han, Y.; Pan, J.; Cao, L. Effects of modified pulmonary rehabilitation on patients with moderate to severe chronic obstructive pulmonary disease: A randomized controlled trail. Int. J. Nurs. Sci. 2017, 4, 219-224. [CrossRef]

181. Zambom-Ferraresi, F.; Cebollero, P.; Gorostiaga, E.M.; Hernandez, M.; Hueto, J.; Cascante, J.; Rezusta, L.; Val, L.; Anton, M.M. Effects of Combined Resistance and Endurance Training Versus Resistance Training Alone on Strength, Exercise Capacity, and Quality of Life in Patients With COPD. J. Cardiopulm. Rehabil. Prev. 2015, 35, 446-453. [CrossRef]

182. ZuWallack, R.; Hashim, A.; McCusker, C.; Normandin, E.; Benoit-Connors, M.L.; Lahiri, B. The trajectory of change over multiple outcome areas during comprehensive outpatient pulmonary rehabilitation. Chron. Respir. Dis. 2006, 3, 11-18. [CrossRef]

183. Zwerink, M.; Effing, T.; Kerstjens, H.A.; van der Valk, P.; Brusse-Keizer, M.; Zielhuis, G.; van der Palen, J. Cost-Effectiveness of a Community-Based Exercise Programme in COPD Self-Management. COPD 2016, 13, 214-223. [CrossRef] [PubMed]

184. Zwerink, M.; van der Palen, J.; Kerstjens, H.A.; van der Valk, P.; Brusse-Keizer, M.; Zielhuis, G.; Effing, T. A community-based exercise programme in COPD self-management: Two years follow-up of the COPE-II study. Respir. Med. 2014, 108, 1481-1490. [CrossRef] [PubMed]

185. Verrill, D.E.; Barton, C.; Beasley, W.; Lippard, W.M. The effects of short-term and long-term pulmonary rehabilitation on functional capacity, perceived dyspnea, and quality of life. Chest 2005, 128, 673-683. [CrossRef] [PubMed]

186. Abd El-Kader, S.M.; Al-Jiffri, O.H.; Ashmawy, E.M.; Gaowgzeh, R.A. Treadmill walking exercise modulates bone mineral status and inflammatory cytokines in obese asthmatic patients with long term intake of corticosteroids. Afr. Health Sci. 2016, 16, 798-808. [CrossRef] [PubMed]

187. Arandelovic, M.; Stankovic, I.; Nikolic, M. Swimming and persons with mild persistant asthma. Sci. World J. 2007, 7, 1182-1188. [CrossRef]

188. Boyd, A.; Yang, C.T.; Estell, K.; Ms, C.T.; Gerald, L.B.; Dransfield, M.; Bamman, M.; Bonner, J.; Atkinson, T.P.; Schwiebert, L.M. Feasibility of exercising adults with asthma: A randomized pilot study. Allergy Asthma Clin. Immunol. Off. J. Can. Soc. Allergy Clin. Immunol. 2012, 8, 13. [CrossRef] [PubMed]

189. Cochrane, L.M.; Clark, C.J. Benefits and problems of a physical training programme for asthmatic patients. Thorax 1990, 45, 345-351. [CrossRef] 
190. Dogra, S.; Kuk, J.L.; Baker, J.; Jamnik, V. Exercise is associated with improved asthma control in adults. Eur. Respir. J. 2011, 37, 318-323. [CrossRef]

191. Franca-Pinto, A.; Mendes, F.A.; de Carvalho-Pinto, R.M.; Agondi, R.C.; Cukier, A.; Stelmach, R.; Saraiva-Romanholo, B.M.; Kalil, J.; Martins, M.A.; Giavina-Bianchi, P.; et al. Aerobic training decreases bronchial hyperresponsiveness and systemic inflammation in patients with moderate or severe asthma: A randomised controlled trial. Thorax 2015, 70, 732-739. [CrossRef]

192. Goncalves, R.C.; Nunes, M.P.T.; Cukier, A.; Stelmach, R.; Martins, M.A.; Carvalho, C.R.F. Effects of an aerobic physical training program on psychosocial characteristics, quality-of-life, symptoms and exhaled nitric oxide in individuals with moderate or severe persistent asthma. Braz. J. Phys. Ther. 2008, 12, 127-135.

193. Haas, F.; Pasierski, S.; Levine, N.; Bishop, M.; Axen, K.; Pineda, H.; Haas, A. Effect of aerobic training on forced expiratory airflow in exercising asthmatic humans. J. Appl. Physiol. 1987, 63, 1230-1235. [CrossRef] [PubMed]

194. Mendes, F.A.; Almeida, F.M.; Cukier, A.; Stelmach, R.; Jacob-Filho, W.; Martins, M.A.; Carvalho, C.R. Effects of aerobic training on airway inflammation in asthmatic patients. Med. Sci. Sports Exerc. 2011, 43, 197-203. [CrossRef] [PubMed]

195. Mendes, F.A.R.; Goncalves, R.C.; Nunes, M.P.T.; Saraiva-Romanholo, B.M.; Cukier, A.; Stelmach, R.; Jacob-Filho, W.; Martins, M.A.; Carvalho, C.R.F. Effects of Aerobic Training on Psychosocial Morbidity and Symptoms in Patients With Asthma A Randomized Clinical Trial. Chest 2010, 138, 331-337. [CrossRef] [PubMed]

196. Bostrom, C.; Elfving, B.; Dupre, B.; Opava, C.H.; Lundberg, I.E.; Jansson, E. Effects of a one-year physical activity programme for women with systemic lupus erythematosus-A randomized controlled study. Lupus 2016, 25, 602-616. [CrossRef] [PubMed]

197. Florian, J.; Rubin, A.; Mattiello, R.; da Fontoura, F.F.; Camargo, J.D.P.; Teixeira, P.J.Z. Impact of pulmonary rehabilitation on quality of life and functional capacity in patients on waiting lists for lung transplantation. $J$. Bras. De Pneumol. 2013, 39, 349-356. [CrossRef] [PubMed]

198. Gaunaurd, I.A.; Gomez-Marin, O.W.; Ramos, C.F.; Sol, C.M.; Cohen, M.I.; Cahalin, L.P.; Cardenas, D.D.; Jackson, R.M. Physical activity and quality of life improvements of patients with idiopathic pulmonary fibrosis completing a pulmonary rehabilitation program. Respir. Care 2014, 59, 1872-1879. [CrossRef] [PubMed]

199. Igarashi, A.; Iwanami, Y.; Sugino, K.; Gocho, K.; Homma, S.; Ebihara, S. Using 6-Min Walk Distance Expressed as a Percentage of Reference to Evaluate the Effect of Pulmonary Rehabilitation in Elderly Patients With Interstitial Lung Disease. J. Cardiopulm. Rehabil. Prev. 2018, 38, 342-347. [CrossRef]

200. Jackson, R.M.; Gómez-Marín, O.W.; Ramos, C.F.; Sol, C.M.; Cohen, M.I.; Gaunaurd, I.A.; Cahalin, L.P.; Cardenas, D.D. Exercise limitation in IPF patients: A randomized trial of pulmonary rehabilitation. Lung 2014, 192, 367-376. [CrossRef]

201. Marcellis, R.; Van der Veeke, M.; Mesters, I.; Drent, M.; De Bie, R.; De Vries, G.; Lenssen, A. Does physical training reduce fatigue in sarcoidosis? Sarcoidosis Vasc. Diffus. Lung Dis. Off. J. Wasog 2015, 32, 53-62.

202. Naz, I.; Ozalevli, S.; Ozkan, S.; Sahin, H. Efficacy of a Structured Exercise Program for Improving Functional Capacity and Quality of Life in Patients With Stage 3 and 4 Sarcoidosis: A RANDOMIZED CONTROLLED TRIAL. J. Cardiopulm. Rehabil. Prev. 2018, 38, 124-130. [CrossRef]

203. Naz, I.; Sahin, H.; Uçsular, F.D.; Yalniz, E. A comparison trial of eight weeks versus twelve weeks of exercise program in interstitial lung diseases. Sarcoidosis Vasc. Diffus. Lung Dis. 2018, 35, 299-307.

204. Perez-Bogerd, S.; Wuyts, W.; Barbier, V.; Demeyer, H.; Van Muylem, A.; Janssens, W.; Troosters, T. Short and long-term effects of pulmonary rehabilitation in interstitial lung diseases: A randomised controlled trial. Respir. Res. 2018, 19, 182. [CrossRef]

205. Sciriha, A.; Lungaro-Mifsud, S.; Fsadni, P.; Scerri, J.; Montefort, S. Pulmonary Rehabilitation in patients with Interstitial Lung Disease: The effects of a 12-week programme. Respir. Med. 2019, 146, 49-56. [CrossRef] [PubMed]

206. Strookappe, B.; Elfferich, M.; Swigris, J.; Verschoof, A.; Veschakelen, J.; Knevel, T.; Drent, M. Benefits of physical training in patients with idiopathic or end-stage sarcoidosis-related pulmonary fibrosis: A pilot study. Sarcoidosis Vasc. Diffus. Lung Dis. Off. J. WASOG 2015, 32, 43-52. 
207. Vainshelboim, B.; Kramer, M.R.; Fox, B.D.; Izhakian, S.; Sagie, A.; Oliveira, J. Supervised exercise training improves exercise cardiovascular function in idiopathic pulmonary fibrosis. Eur. J. Phys. Rehabil. Med. 2017, 53, 209-218. [PubMed]

208. Vainshelboim, B.; Oliveira, J.; Fox, B.D.; Soreck, Y.; Fruchter, O.; Kramer, M.R. Long-term effects of a 12-week exercise training program on clinical outcomes in idiopathic pulmonary fibrosis. Lung 2015, 193, 345-354. [CrossRef] [PubMed]

209. Vainshelboim, B.; Oliveira, J.; Yehoshua, L.; Weiss, I.; Fox, B.D.; Fruchter, O.; Kramer, M.R. Exercise training-based pulmonary rehabilitation program is clinically beneficial for idiopathic pulmonary fibrosis. Respir. Int. Rev. Thorac. Dis. 2014, 88, 378-388. [CrossRef] [PubMed]

210. Cazzola, M.; Calzetta, L.; Bettoncelli, G.; Novelli, L.; Cricelli, C.; Rogliani, P. Asthma and comorbid medical illness. Eur. Respir. J. 2011, 38, 42-49. [CrossRef] [PubMed]

211. King, C.S.; Nathan, S.D. Idiopathic pulmonary fibrosis: Effects and optimal management of comorbidities. Lancet Respir. Med. 2017, 5, 72-84. [CrossRef]

212. Fabbri, L.M.; Boyd, C.; Boschetto, P.; Rabe, K.F.; Buist, A.S.; Yawn, B.; Leff, B.; Kent, D.M.; Schünemann, H.J. How to integrate multiple comorbidities in guideline development: Article 10 in integrating and coordinating efforts in COPD guideline development. An official ATS/ERS workshop report. Proc. Am. Thorac. Soc. 2012, 9, 274-281. [CrossRef]

213. Schulz, K.F.; Altman, D.G.; Moher, D. CONSORT 2010 statement: Updated guidelines for reporting parallel group randomised trials. BMC Med. 2010, 8, 18. [CrossRef] [PubMed]

214. Hsu, C.-Y.; Hsieh, P.-L.; Hsiao, S.-F.; Chien, M.-Y. Effects of exercise training on autonomic function in chronic heart failure: Systematic review. Biomed Res. Int. 2015, 2015, 8. [CrossRef] [PubMed]

215. Cornelis, N.; Nassen, J.; Buys, R.; Fourneau, I.; Cornelissen, V. The Impact of Supervised Exercise Training on Traditional Cardiovascular Risk Factors in Patients With Intermittent Claudication: A Systematic Review and Meta-Analysis. Eur. J. Vasc. Endovasc. Surg. 2019, 58, 75-87. [CrossRef] [PubMed]

216. Kachur, S.; Chongthammakun, V.; Lavie, C.J.; De Schutter, A.; Arena, R.; Milani, R.V.; Franklin, B.A. Impact of cardiac rehabilitation and exercise training programs in coronary heart disease. Prog. Cardiovasc. Dis. 2017, 60, 103-114. [CrossRef] [PubMed]

217. Lavie, C.J.; Arena, R.; Swift, D.L.; Johannsen, N.M.; Sui, X.; Lee, D.-c.; Earnest, C.P.; Church, T.S.; O’Keefe, J.H.; Milani, R.V. Exercise and the cardiovascular system: Clinical science and cardiovascular outcomes. Circ. Res. 2015, 117, 207-219. [CrossRef]

218. Hansen, D.; Dendale, P.; Coninx, K.; Vanhees, L.; Piepoli, M.F.; Niebauer, J.; Cornelissen, V.; Pedretti, R.; Geurts, E.; Ruiz, G.R. The European Association of Preventive Cardiology Exercise Prescription in Everyday Practice and Rehabilitative Training (EXPERT) tool: A digital training and decision support system for optimized exercise prescription in cardiovascular disease. Concept, definitions and construction methodology. Eur. J. Prev. Cardiol. 2017, 24, 1017-1031. [PubMed]

219. American Association of Cardiovascular Pulmonary Rehabilitation. Guidelines for Pulmonary Rehabilitation Programs; Human Kinetics: Stanningley, UK, 2011.

220. Troosters, T.; Van Remoortel, H. Pulmonary rehabilitation and cardiovascular disease. Semin. Respir. Crit. Care Med. 2009, 30, 675-683. [CrossRef]

221. Hansen, D.; Piepoli, M.F.; Doehner, W. The importance of rehabilitation in the secondary prevention of cardiovascular disease. Eur. J. Prev. Cardiol. 2019, 26, 273-276. [CrossRef]

222. American College of Sports Medicine. ACSM's Guidelines for Exercise Testing and Prescription; Lippincott Williams \& Wilkins: Philadelphia, PA, USA, 2013.

223. Piepoli, M.F.; Corra, U.; Benzer, W.; Bjarnason-Wehrens, B.; Dendale, P.; Gaita, D.; McGee, H.; Mendes, M.; Niebauer, J.; Zwisler, A.-D.O. Secondary prevention through cardiac rehabilitation: From knowledge to implementation. A position paper from the Cardiac Rehabilitation Section of the European Association of Cardiovascular Prevention and Rehabilitation. Eur. J. Cardiovasc. Prev. Rehabil. 2010, 17, 1-17. [CrossRef]

224. Wenger, N.K. Current status of cardiac rehabilitation. J. Am. Coll. Cardiol. 2008, 51, 1619-1631. [CrossRef]

(C) 2019 by the authors. Licensee MDPI, Basel, Switzerland. This article is an open access article distributed under the terms and conditions of the Creative Commons Attribution (CC BY) license (http://creativecommons.org/licenses/by/4.0/). 